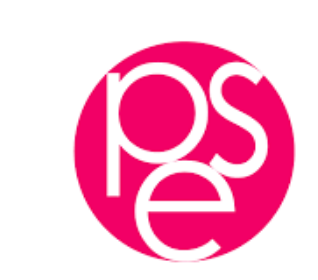

PARISSCHOOLOFECONOMICS
ECOLED'ECONOMIEDEPARIS

WORKING PAPER Nº $2022-03$

Voting in Shareholders Meetings

\author{
Laurent Bouton \\ Aniol Llorente-Saguer \\ Antonin Macé \\ Dimitrios Xefteris
}

JEL Codes: D72, G3

Keywords: Information Aggregation, Strategic Voting, Shareholder Meetings, Corporate Governance, One-person-one-vote, One-share-one-vote

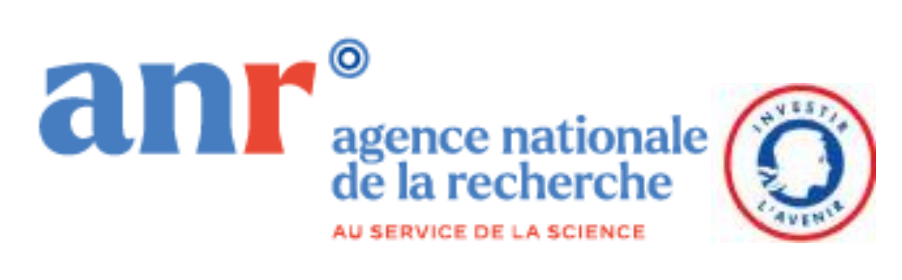




\title{
Voting in Shareholders Meetings*
}

\author{
Laurent Bouton ${ }^{\dagger}$ \\ Aniol Llorente-Saguer ${ }^{\ddagger}$ \\ Antonin Macé ${ }^{\S}$ \\ Dimitrios Xefteris
}

November 16, 2021

\begin{abstract}
This paper studies the informational efficiency of voting mechanisms in shareholder meetings. When the management cannot affect the proposal being voted on, we show that voting mechanisms are more efficient when their ballot space is richer. Moreover, efficiency requires full divisibility of the votes. When the management has agenda power, we uncover a novel trade-off: more efficient mechanisms provide worse incentives to select good proposals. This negative effect can be large enough to wash out the higher voting efficiency of even the most efficient mechanisms.

JEL Classification: D72, G3

Keywords: Information Aggregation, Strategic Voting, Shareholder Meetings, Corporate Governance, One-person-one-vote, One-share-one-vote.
\end{abstract}

\footnotetext{
${ }^{*}$ We greatly benefited from the insights, comments, and suggestions of Alon Brav, Micael Castanheira, Florian Ederer, Garance Genicot, German Gieczewski, Alessandro Lizzeri, Kirill Pogorelskiy, Jason Sturgess, Moqi Xu, and seminar and conference participants at Queen Mary University of London, UC-Berkeley, University of Warwick, University of Cyprus, Universite Paris 1, University of Leicester, the Columbia University Political Economy Conference, and the 3rd ETH Workshop on Democracy: Theoretical Political Economy. This project has received funding from the European Research Council (Laurent Bouton) under the European Union's Horizon 2020 research and innovation programme (grant agreement No 637662).

${ }^{\dagger}$ Department of Economics, Georgetown University, NBER, and CEPR. E-mail: boutonllj@gmail.com

${ }^{\ddagger}$ School of Economics and Finance, Queen Mary University of London, and CEPR. Email: a.llorente-saguer@qmul.ac.uk

${ }^{\S}$ CNRS and Paris School of Economics. Email: antonin.mace@gmail.com.

`Department of Economics, University of Cyprus. Email: xefteris.dimitrios@ucy.ac.cy.
} 


\section{Introduction}

Shareholders typically hold different number of shares. ${ }^{1}$ Corporations thus face a non-trivial choice of how to allocate voting rights. How should the number of votes a shareholder has depend on the number of shares she owns? Nowadays, most corporations follow the one-share-one-vote principle and attach one vote to each share. But recent trends are towards more progressive systems in which some shareholders are awarded an oversized share of the voting rights, e.g., dual-class stock (see, e.g., Adams and Ferreira 2008, and Hayden and Bodie 2008). By contrast, in the 19th century, deviations from the one-share-one-vote principle were more regressive. ${ }^{2}$

We consider a specific facet of the question of the allocation of voting rights. Our focus is on how it affects information aggregation, and hence the desirability of the outcome for shareholders. ${ }^{3}$ Crucial to this question is how different voting mechanisms affect both shareholders' decisions at the meeting and the management incentives before the meeting.

In the first part of the paper, we focus on the strategic behavior of shareholders at the meeting, taking the management proposal as given. ${ }^{4}$ Since we allow for heterogeneity in shareholdings, shareholders differ in how much they care about the performance of the firm, and hence about the decision at the meeting. Yet, in line with most of the literature on shareholders meetings, we focus on cases in which (most) shareholders have state-contingent preferences: if the management proposal increases the value of the firm, they are all better off if approved. Otherwise, they prefer to stick to the status quo. The issue is that, at the time of the vote, shareholders are only imperfectly informed about the desirability of the proposal. Moreover, as is the case in practice, some shareholders may be more precisely informed than

\footnotetext{
${ }^{1}$ For instance, $96 \%$ of the firms in a representative sample of US firms have at least one blockholder, i.e., a shareholder who own more than $5 \%$ of the outstanding shares (Holderness 2009). Similarly, as Edmans and Holderness (2017) discuss, many institutional investors hold less than $5 \%$ of the outstanding shares of a given firm but still hold a very substantial number of shares. By contrast, retail shareholders hold much fewer shares of a given firm. Moreover, the dispersion of shareholdings among retail shareholders is substantial (Brav et al. 2019).

${ }^{2}$ In the 19th century most corporations used to restrict the voting power of any given shareholder; with many of them employing one-person-one-vote mechanisms (see, e.g., Hansmann and Pargendler 2013).

${ }^{3}$ The heterogeneity in shareholdings, and hence the question of which voting mechanism to use, has been overlooked by most of the literature on voting in shareholders meetings. Bar-Isaac and Shapiro (2020) is a notable exception.

${ }^{4}$ As discussed in Christoffersen et al. (2007) and Yermack (2010), management's proposals include, e.g., possible mergers and acquisitions, the issuance of new shares, the sale of the firm, amendments to governance procedures, changes in voting rights of directors, and new compensation package for directors.
} 
others, and their information precision need not be publicly observed.

We establish two main results for this case with an exogenous proposal. First, we compare mechanisms with a finite ballot space in terms of their information aggregation efficiency (i.e., the likelihood of selection of the best alternative). We show that, for any distribution of shareholdings and any informational asymmetry among shareholders, a voting mechanism with a richer ballot space dominates a voting mechanism with a poorer one. This is because strategic shareholders can use the richer ballot space to adjust their impact on the voting outcome to the accuracy of their information.

Second, we show that efficiency requires votes to be fully divisible. This is so because, under any mechanism with fully divisible votes (the continuous voting mechanisms), such as $1 S 1 \mathrm{~V}-\mathrm{D}$ and $1 \mathrm{P} 1 \mathrm{~V}-\mathrm{D},{ }^{5}$ all shareholders have the ability to reveal their information fully, even if they hold only a small number of shares. This second result highlights that, from an information aggregation standpoint, the distribution of votes across shareholders becomes irrelevant when votes are fully divisible (and all shareholders have at least one vote). Thus, as long as votes are made fully divisible, decisions about how to distribute voting power across shareholders can focus on other facets of the issue (e.g., fairness, effects on takeovers or on the firms' choice of ownership and financing). The efficiency of mechanisms with fully divisible votes is robust to the presence of (i) partisan shareholders, (ii) super-majority thresholds, (iii) ambiguity about the information technology of other shareholders, and (iv) endogenous acquisition of information by shareholders.

In the second part of the paper, we explore how voting mechanisms affect the management's incentives to shape the proposal before the meeting. We focus on the extensive margin: the management decides whether to put a proposal to the vote after observing a signal about its quality. If the management blocks the proposal, then the status quo remains. The properties of a voting mechanism then depend both on (i) selection (i.e., the incentives it provides the management to select good proposals and veto bad ones), and (ii) voting efficiency (i.e., the quality of information aggregation at the meeting).

Following the corporate governance and finance literature, we allow for conflict between shareholders and the management (see Tirole 2005 and references therein). We consider two specific dimensions of conflict. First, the manager may be mis-

\footnotetext{
${ }^{5}$ Under $1 S 1 \mathrm{~V}$-D shareholders can, for every share they own, allocate any fraction of point between 0 and 1 in favor or against the management's proposal. The proposal is approved if and only if the total number of points in favor is larger than the ones against. The $1 P 1 V-D$ is similar except that every shareholder has only one point to allocate.
} 
aligned, in the sense that she wants the proposal to be adopted even when it is undesirable for the shareholders. ${ }^{6}$ Second, the manager may incur a (reputation) cost if a proposal is rejected at the shareholder meeting. ${ }^{7}$

We uncover a trade-off between selection and voting efficiency underlying the comparison of continuous voting mechanisms and other mechanisms with lower voting efficiency (we focus on the comparison of $1 P 1 \mathrm{~V}-\mathrm{D}$ and $1 P 1 \mathrm{~V}$ for the sake of expositional clarity). ${ }^{8}$ We identify two different effects of the voting mechanism on the behavior of the manager. First, higher voting efficiency reduces the incentives of an aligned manager to veto any reform because she trusts that the shareholders will choose correctly with high probability. Therefore, the proposals she puts forward under $1 P 1 \mathrm{~V}-\mathrm{D}$ are, on average, inferior to the proposals she puts forward under $1 P 1 \mathrm{~V}$. This better selection of proposals under $1 P 1 \mathrm{~V}$ is not strong enough to compensate for the higher voting efficiency of $1 P 1 \mathrm{~V}-D$.

Second, when rejection of the proposal at the meeting is costly for the manager, she may have incentives to veto the proposal even when misaligned. We show that, in equilibrium, the misaligned manager also vetoes bad proposals with some probability; a selection of proposals that is beneficial to shareholders. We then find that the incentives to veto bad proposals can be stronger under $1 \mathrm{P} 1 \mathrm{~V}$ because the shareholders are more likely to reject the proposal if it reaches the meeting. In some cases, the difference in selection of proposals between $1 \mathrm{P} 1 \mathrm{~V}-\mathrm{D}$ and $1 \mathrm{P} 1 \mathrm{~V}$ can be sufficiently strong to compensate for the higher voting efficiency of $1 P 1 \mathrm{~V}-D$. Then, shareholders are better off under $1 P 1 V$ than $1 P 1 V$ - D. This result suggests

\footnotetext{
${ }^{6}$ For instance, as discussed in Becht et al. (2016), there is empirical evidence showing that a substantial share of corporate acquisisions are associated with negative returns for acquirer shareholders. One explanation is that, in the case of M\&A, managerial wealth and shareholder wealth are decoupled (Grinstein and Hribar 2004; Harford and Li 2007; Fu, Lin, and Officer 2013). Another area of conflict is Say-on-Pay proposals (Cunat, Gine, and Guadalupe 2016). Finally, Bach and Metzger (2016) and Babenko, Choi, and Sen (2019) find evidence that managers manipulate the voting process to increase the success rate of their proposals. Those manipulations appear to be value-destroying. For a more thorough discussion of conflict between managers and shareholders see, e.g., Tirole (2005, Chapter 1).

${ }^{7}$ Becht et al. (2016) and Gantchev and Giannetti (2020) mention the existence of such reputational costs but we are not aware of direct evidence. However, there is suggestive evidence. For instance, Cai, Garner, and Walking (2009) and Aggarwal, Dahiya, and Prabhala (2017) find that dissent votes in uncontested director elections have negative consequences for both directors and executives. The rejection of a management proposal at the meeting could have similar implications for the management. Also, Li et al. (2018) find that managers trying to acquire another corporation implement various strategies to avoid shareholder voting on the acquisition, and hence the risk of an embarassing rebuke at the shareholder meeting.

${ }^{8}$ Under $1 P 1 \mathrm{~V}$, shareholders are endowed with only one point that they can cast in favor or against the proposal. The proposal is approved if the number of points in favor is larger than a predetermined (super-)majority threshold ( $50 \%$ in the case of simple majority).
} 
one possible explanation of why, in practice, corporations do not make votes fully divisible.

\subsection{Related Literature}

There is a large empirical literature studying the effect of shareholder voting on firms performance and management's behavior. Overall, this literature finds that control by shareholders (i) affects positively firms' performance, and (ii) is key to provide proper incentives to the management. ${ }^{9}$ The specifics of corporate governance rules and procedures appear to play an important role. As summarized by Yermack (2010, p. 106): research that studies the "[...] general effects of voting restrictions on firm value and performance, often [finds] that firms perform worse when the shareholder franchise is curtailed [...]. Notable recent papers in this large literature include Gompers et al. (2003), which examines a range of takeover defenses and voting restrictions; Bebchuk and Cohen (2005) and Faleye (2007), both of which focus on staggered boards; and Gompers et al. (2009), which studies dual-class voting structures." 10 More directly connected to us, Burkart and Lee (2008) review the theoretical literature on the one-share-one-vote principle and highlight three classes of effects of the security-voting structure (aka allocation of voting rights): effects on takeovers, effects on incentives of blockholders, and effects on the firms' choice of ownership and financing. ${ }^{11}$ We complement that literature by highlighting the effects of voting mechanisms on the quality of decisions at shareholders meetings, and how that shapes managers' incentives. Particularly relevant for that literature is our result that, as long as votes are made fully divisible, decisions about how to distribute voting power across shareholders can focus on other facets of the issue.

The exogenous proposal part of our paper contributes to the literature on infor-

\footnotetext{
${ }^{9}$ For instance, Appel, Gormley, and Keim (2016) find that even passive investors affect positively firms' longer-term performance through voting; Fos, Li, and Tsoutsoura (2018) find that the proximity to an election affects the behavior of directors; Li et al. (2018) finds that, in the US, shareholder voting help mitigate agency problems that plague corporate acquisitions; Becht et al. (2016) find that shareholder voting has a substantial positive effect on the quality of acquisitions by firms in the UK; Richardson (2000) finds a positive relationship between information asymmetry between managers and shareholders, which impedes the ability of the latter to control efficiently the former, and earnings management (i.e., funky accounting by managers); Cai et al. (2006) and Aggarwal et al. (2019) focus on directors elections in US firms and find that even uncontested elections affect the firms and directors in various dimensions; Conyon and Sadler (2010), Ferri and Maber (2012), and Alissa (2015) study the effects of say-on-pay votes in the UK and find that they constrain the size and the structure of top managers' pay.

${ }^{10}$ Note however that Frankenreiter et al. (2021) have recently cast some serious doubts on the reliability of the datasets used in this literature.

${ }^{11}$ See Adams and Ferreira (2008) for a survey of the empirical literature on the topic.
} 
mation aggregation in committees and elections with exogenous alternatives (see, e.g., Austen-Smith and Banks 1996, Feddersen and Pesendorfer 1996, 1997, 1998, Fey 1997, Myerson 1998, Mandler 2012; Bouton and Castanheira 2012, Bhattacharya 2013, McMurray 2013, Bouton et al. 2018, Barelli et al. 2019, Herrera et al. 2019, and Bar-Isaac and Shapiro 2020). The closest paper to ours is Bar-Isaac and Shapiro (2020). They consider a setup with one blockholder who owns many shares, and many retail shareholders who own one share each. We generalize their model, and their result of the desirability of partial abstention, by considering (i) any distribution of shares among shareholders, including but not limited to multiple blockholders, (ii) any correlation between shareholdings and information precision, and (iii) imperfect information about shareholders' information precision. Three other key differences are that, first, we compare the properties of various voting mechanisms in our generalized setup. This includes the identification of the optimal voting mechanisms for any shareholdings. Second, we study the information aggregation properties of voting mechanisms taking into account of their effects on the management's incentives. That allows us to identify situations in which partial abstention, through its effects on the management's incentives, is detrimental to information aggregation. Finally, they identify share trading before the meeting as a possible factor reducing the desirability of partial abstention. As we discuss in Section 6, share trading a la Bar-Isaac and Shapiro does not eliminate the desirability of partial abstention when the quality of information of each shareholder is her private information.

Our result that, from an information aggregation standpoint, the distribution of votes across shareholders becomes irrelevant when votes are fully divisible and voters are strategic stands in stark contrast with the predictions of sincere-voting models which prescribe that voting power should be proportional to information precision for full-information equivalence to be achieved (see, e.g., Nitzan and Paroush 1982 and Azrieli 2018). As we show, in settings of applied interest where it is hard to assign voting rights proportionally to information precisions (e.g., because they are privately observed), vote divisibility is enough to guarantee the existence of an efficient equilibrium outcome. This is because, when given the flexibility, strategic voters endogenously choose the optimal voting power.

The endogenous proposal part of our paper contributes to the literature on information aggregation with endogenous alternatives. We are only aware of two papers exploring that question: Henry (2008), and Bond and Eraslan (2010). The latter is the closest to our paper. It stresses the importance of endogenizing the proposal 
when one studies the information properties of a voting system. But, given our focus on shareholders meetings, our model and insights are quite different than those in Bond and Eraslan (2010). Crucially, we permit the distribution of votes to depend on the shareholding structure. This allows us to compare mechanisms which differ along three dimensions: the balance of power across shareholders, the divisibility of the votes, and the super-majority threshold. By contrast, Bond and Eraslan (2010) focus on the super-majority threshold comparing one-person-one-vote mechanisms such as majority and unanimity. Moreover, by comparing mechanisms which admit an unambiguous ranking in terms of voting efficiency (richer vs. poorer ballot space), we uncover a general tradeoff between voting efficiency and selection incentives that is not possible to detect when limiting attention to classes of one-person-one-vote mechanisms. ${ }^{12}$ Also, we focus on the extensive margin of action for the manager, instead of the intensive margin in Bond and Eraslan (2010). That allows us to show that selection incentives can upset the welfare ordering of two mechanisms even if the manager only controls the agenda and has limited power to adjust the details of the proposal.

\section{Model}

In this section we present our baseline model. In Section 6 we provide a discussion of some of the key assumptions, including restrictions on communication, share trading, and vote trading. In Appendix D, we prove the robustness of our results to various extensions of the model.

Consider a firm with a set $N=\{1,2, \ldots, n\}$ of shareholders, with $n>2$, and $m \geq n$ shares. A shareholder $i \in N$ holds $d_{i} \in \mathbb{N}$ shares, with $\sum_{i \in N} d_{i}=m$. Let $d=\left(d_{1}, d_{2}, \ldots, d_{n}\right)$ be the vector of the shares among the $n$ shareholders, $h_{i}=\frac{d_{i}}{m}$ be the fraction of shares held by shareholder $i$, and $h=\left(h_{1}, h_{2}, \ldots, h_{n}\right)$ the vector of the fraction of shares held by the $n$ shareholders.

At the shareholders meeting, they have to choose, through voting, whether to approve a proposal by the management, $A$, or keep the status quo, $B$. We denote the set of alternatives by $O=\{A, B\}$. For now, we assume that the proposal is exogenously given. In Section 5 we allow the management to shape the proposal.

Shareholders are uncertain about the quality of the proposal. There are two

\footnotetext{
${ }^{12}$ Super-majority rules-a prominent class of $1 \mathrm{P} 1 \mathrm{~V}$ mechanisms-do not admit a clear ranking in terms of voting efficiency: each super-majority rule is optimal under certain informational assumptions (see, e.g., Maug and Rydqvist 2009).
} 
states of the world, $\omega \in \Omega=\{\alpha, \beta\}$ that are unobserved at the time of the vote. For the sake of simplicity, we assume that the states are equiprobable.

Preferences. While shareholders do not necessarily have the same stakes, they all agree that the proposal is good in state $\alpha$ but bad in state $\beta$ :

$$
\begin{aligned}
& u_{i}(A \mid \alpha)=h_{i}, u_{i}(A \mid \beta)=-h_{i}, \\
& u_{i}(B \mid \alpha)=u_{i}(B \mid \beta)=0 .
\end{aligned}
$$

Information. Before the meeting, each shareholder $i$ receives a signal $s_{i} \in S:=$ $[0,1]$ distributed according to a shareholder-specific distribution function, $F_{i}(\cdot \mid \omega)$, with density $f_{i}(\cdot \mid \omega) .{ }^{13}$ Conditional on the state, signals are drawn independently. The type of shareholder $i$ after the draw of the signal is $t_{i}=\frac{f_{i}\left(s_{i} \mid \alpha\right)}{f_{i}\left(s_{i} \mid \beta\right)} \in T_{i}=\left[\delta_{i}, 1 / \delta_{i}\right]{ }^{14}$ We make the following assumption about the signal technology:

Assumption 1 (Strong MLRP and Bounded Support) For every shareholder $i \in N, t_{i}$ is strictly increasing in $s_{i}$ and there exists $\delta_{i} \in(0,1)$ such that $\frac{f_{i}(0 \mid \alpha)}{f_{i}(0 \mid \beta)}=\delta_{i}$ and $\frac{f_{i}(1 \mid \alpha)}{f_{i}(1 \mid \beta)}=1 / \delta_{i}$.

The first part of Assumption 1 means that shareholders who receive higher signals attach a larger probability to the state of the world being state $\alpha$, while the second part means that there is no shareholder with arbitrarily precise information about the state of the world. This assumption allows for various structures of shareholders' information technology. For instance, it allows for arbitrarily large differences in the (expected) information quality of two shareholders, and for any type of correlation between (expected) information quality of a shareholder and the number of shares she owns.

Voting Mechanisms. We consider a broad class of voting mechanisms. A voting mechanism $V$ associates to any share distribution $d$ a voting rule $V(d)=X$, where $X=\left(X_{1}, X_{2}, \ldots, X_{n}\right)$ with $X_{i} \subseteq \mathbb{R}$, and is such that each shareholder $i \in N$ chooses $x_{i} \in X_{i}$ and the outcome is:

$$
G^{w}(x)=\left\{\begin{array}{lll}
A & \text { if } & \sum_{i \in N} x_{i}>0 \\
A B & \text { if } & \sum_{i \in N} x_{i}=0 \\
B & \text { if } & \sum_{i \in N} x_{i}<0
\end{array}\right.
$$

\footnotetext{
${ }^{13}$ In Appendix D, we endogenize the acquisition of information by shareholders.

${ }^{14}$ The type $t_{i}$ of a shareholder summarizes the strategically relevant information contained in her signal $s_{i}$, which corresponds to the relative likelihood of state $\alpha$ once the signal has been received.
} 
where $A B$ denotes the fair lottery between $A$ and $B .{ }^{15}$

This class of voting mechanisms nests most popular mechanisms, including some that are closer to one-person-one-vote procedures, like majority rule, and some that distribute voting power depending on the exact number of shares that each shareholder holds. For instance, the widely studied one-person-one-vote mechanism, $V^{1 P 1 V}$, is such that $V^{1 P 1 V}(d)=\times_{i \in N}\{-1,1\}$. Each shareholder has to choose among two ballots, voting for $A$ (action 1 ) or voting for $B$ (action -1). ${ }^{16}$ The one-share-onevote mechanism, $V^{1 S 1 V}$, is such that $V^{1 S 1 V}(d)=\times_{i \in N}\left\{-d_{i},-d_{i}+1,-d_{i}+2, \ldots, d_{i}\right\}$. Each shareholder $i$ can choose from a rich set of ballots: either fully supporting $A$ (action $d_{i}$ ), fully supporting $B$ (action $-d_{i}$ ), or intermediate intensities of support (integer numbers between $-d_{i}$ and $\left.d_{i}\right) .{ }^{17}$ Under both mechanisms, the proposal is approved if the total support for $A$ is strictly larger than the total support for $B$, and ties are broken randomly. Due to their empirical relevance in shareholders meetings and the literature, we will often mention those two mechanisms and their specific properties.

Variations of $1 \mathrm{P} 1 \mathrm{~V}$ and $1 S 1 \mathrm{~V}$ in which votes are fully divisible will also prove of particular interest. First, the one-person-one-vote with divisible votes mechanism, $V^{1 P 1 V-D}$, is such that $V^{1 P 1 V-D}(d)=\times_{i \in N}[-1,1]$. Second, the one-share-one-vote with divisible votes mechanism, $V^{1 S 1 V-D}$, is such that $V^{1 S 1 V-D}(d)=\times_{i \in N}\left[-d_{i}, d_{i}\right]$. Under both mechanisms, the vote divisibility is maximal: for each of their votes, shareholders can choose any intermediate intensity of support, not only integers. The ballot space is thus richer under $1 P 1 \mathrm{~V}$-D than $1 P 1 \mathrm{~V}$ and under $1 S 1 \mathrm{~V}$-D than $1 S 1 V$.

The $1 P 1 V$ - $D$ and $1 S 1 V$ - $D$ mechanisms belong to the class of continuous voting mechanisms:

Definition 1 A voting mechanism $V$ with associated rules $V(d)=X$ is a continuous voting mechanism if for every $d$ there exists $\left(\psi_{i}\right)_{i \in N} \in \times_{i \in N} i n t\left(X_{i}\right)$, such that $\sum_{i \in N} \psi_{i}=0$.

\footnotetext{
${ }^{15}$ Note that our setup encompasses rules with super-majority threshold requirements. For instance, a rule where $A$ passes whenever $\sum_{i \in N} x_{i}>w$ for $x_{i} \in Y_{i}$ and $w \in \mathbb{R}$ can be considered in our setup by setting $X_{i}=\left\{x_{i}-\frac{w}{n} \mid x_{i} \in Y_{i}\right\}$ for each shareholder $i$, as $\sum_{i \in N} x_{i}>w \Leftrightarrow$ $\sum_{i \in N}\left(x_{i}-\frac{w}{n}\right)>0$.

${ }^{16}$ We do not allow for abstention for simplicity of exposure. However, as it will become clear in section 4 , all our results are robust to allowing for abstention.

${ }^{17}$ Note that in this definition of one-share-one-vote, shareholders can partially abstain, i.e., can cast only some of their shares in favor of one of the alternatives. As discussed in Bar-Isaac and Shapiro (2020), in practice such partial abstention may not be feasible for some shareholders.
} 
The class of continuous voting mechanisms is such that (i) all shareholders have access to a continuous ballot space, and (ii) the ballot space allows for a tie in which each voter can increase and decrease the net vote total by any arbitrarily small degree.

While we formally consider simple capital structures consisting only of common shares, our model also accommodates dual class structures with both common shares (which carry voting rights) and preferential shares (which do not carry voting rights). Indeed, if each shareholder holds some common shares, and some preferential ones, then, again, the voting rules should depend only on the distribution of the common shares, leaving the model intact. ${ }^{18}$

It will be useful to distinguish between cases in which there is a shareholder who can always affect the outcome independently of the choices of the other shareholders, and the more interesting cases in which such a player is not present. In particular, we say that there is no decisive shareholder if, for a given voting mechanism $V$ and a share distribution $d$, there exists a strategy profile such that $A(B)$ wins with certainty and the outcome cannot be affected by any individual deviation. In all other cases, we say that a decisive shareholder exists.

Strategies and Equilibrium Concept. For each voting rule $X$ and each shareholder $i \in N$, a strategy is a function $\sigma_{i}: T_{i} \rightarrow \Delta\left(X_{i}\right)$. As it is standard in the literature, $\Delta\left(X_{i}\right)$ is the set of all probability distributions on $X_{i}$. When $\sigma_{i}$ is a pure strategy, we sometimes abuse notation and denote by $\sigma_{i}\left(t_{i}\right)$ the action $x$ that the shareholder picks with probability 1 . Since $\sigma_{i}$ can be a mixed strategy, it is useful to distinguish the random variable, $\sigma_{i}$, from a potential realization, $\widehat{\sigma}_{i}$ : we say that $\widehat{\sigma}_{i}$ is a potential realization of $\sigma_{i}$ if and only if $\widehat{\sigma}_{i}$ belongs in the support of $\sigma_{i}$. Consequently, when $\sigma_{i}$ is a pure strategy we have $\sigma_{i}\left(t_{i}\right)=\widehat{\sigma}_{i}\left(t_{i}\right)$ for every $t_{i} \in T_{i}$.

We focus on (interim) Bayesian Nash Equilibria (BNE) such that equilibrium strategies are best responses at the interim stage (when each shareholder knows her own type).

\section{Welfare Benchmarks}

We consider two different welfare benchmarks, which serve different purposes: one is to assess the efficiency of a mechanism, and the other to compare mechanisms

\footnotetext{
${ }^{18}$ In this more general case one should allow $h_{i}$ to depend on both kinds of shareholdings. As it will be made clear in our analysis, the exact value of $h_{i}$ does not alter shareholder $i$ 's incentives, and hence the equilibria of the game.
} 
which are not efficient.

Our efficiency benchmark corresponds to the preferred outcome of shareholders when they have access to all the information dispersed in the electorate (i.e., if they were able to observe the signal profile). In that case, shareholders would prefer the alternative that is most likely to match the state of the world, conditional on the available information:

Definition 2 Given a vector of signals $s=\left(s_{1}, s_{2}, \ldots, s_{n}\right)$, the efficient outcome, $E$, is equal to $A$ if $\operatorname{Pr}(\alpha \mid s)>1 / 2, B$ if $\operatorname{Pr}(\beta \mid s)>1 / 2$, and $A B$ otherwise.

This leads to a natural implementation notion:

Definition 3 Given a voting rule $X$, a BNE $\sigma=\left(\sigma_{1}, \ldots, \sigma_{n}\right)$ is efficient if $G^{w}\left(\sum_{i \in N} \widehat{\sigma}_{i}\left(t_{i}\right)\right)=E$ for every $t \in \Pi_{i \in N} T_{i}$ and every potential realization $\widehat{\sigma}(t)$ of the random variables $\left\{\sigma_{1}\left(t_{1}\right), \sigma_{2}\left(t_{2}\right), \ldots\right\}$. A voting mechanism $V$ implements the efficient outcome in equilibrium, if for every $d, V(d)$ admits an efficient BNE.

Our second welfare benchmark corresponds to the preferred outcome of shareholders when they know the state of the world:

Definition 4 Given the state of the world $\omega$, the correct outcome is A in state $\alpha$, and $B$ in state $\beta$.

The two welfare benchmarks are fully compatible: when the state of the world is unobservable, then the alternative that is most likely the correct outcome given the available information coincides with the efficient outcome. Moreover, in our common value environment, both these welfare benchmarks are aligned with utilitarian principles. The correct outcome is the utilitarian outcome (i.e. the alternative that maximizes the sum of ex-post utilities), and the efficient outcome is the outcome most likely to be the utilitarian one given all the shareholders' information.

We then compare the performance of voting mechanisms focusing on the ex-ante (i.e. before the state of the world and types are drawn) probability with which they implement the correct decision, considering both the best equilibria (in terms of selecting the correct outcome), and the worst ones:

Definition 5 Voting mechanism $V$ dominates voting mechanism $V^{\prime}$ given a share distribution d if (i) for every BNE of $V^{\prime}(d)$, there is a BNE of $V(d)$ such that the exante probability of implementing the correct outcome is higher under $V$ than $V^{\prime}$; and 
(ii) for every $B N E$ of $V(d)$, there is a $B N E$ of $V^{\prime}(d)$ such that the ex-ante probability of implementing the correct outcome is lower under $V^{\prime}$ than $V$. If, moreover, either (i) or (ii) (or both) hold strictly, we say that $V$ strictly dominates $V^{\prime}$.

Voting mechanisms typically admit multiple equilibria. Therefore, assessing the potential performance of a mechanism considering only the best (worst) equilibrium, in terms of the ex-ante probability of selecting the correct alternative, might be overly optimistic (pessimistic). For this reason, we opt for a comparative criterion that combines both the best and the worst possible equilibrium outcomes. We say that a mechanism dominates another if and only if the former is superior to the latter in both dimensions. This is particularly important in our case because, as we will illustrate below, mechanisms with richer ballot spaces allow for both better and worse outcomes compared to mechanisms with poorer ballot spaces. Hence, focusing only on the best (worst) equilibrium of each mechanism might not be very informative with respect to the range of equilibrium performances.

Note that we do not limit attention to equilibria in undominated strategies. Importantly, we will show next that both the best and the worst equilibrium are typically not in dominated strategies. Hence, in most cases, the comparison between voting mechanisms cannot become any sharper by focusing on this oft-used refinement in voting games (see, e.g., Bouton and Castanheira 2012).

\section{Equilibrium Analysis: Exogenous Proposal}

In this section, we analyze the equilibrium performances of various voting mechanisms when the manager is passive, i.e., the proposal is exogenously given. We split the section in two parts. First, we compare the information aggregation properties of finite voting mechanisms, i.e., mechanisms with finite ballot spaces such as $1 P 1 \mathrm{~V}$ and $1 S 1 \mathrm{~V}$. Second, we focus on the informational efficiency of all types of voting mechanisms. In Appendix D, we explore the robustness of our results when we relax the assumption that all shareholders agree whether the proposal should pass by allowing for partisan shareholders, and when we endogenize the acquisition of information by shareholders.

We first introduce the following Lemma, which is not only useful to prove some of our key results, but also to understand the benefits and pitfalls of a mechanism with a richer ballot space.

Lemma $1 \operatorname{Pr}(\alpha \mid s)>\frac{1}{2} \Leftrightarrow \sum_{i \in N} \ln \left(t_{i}\right)>0$. 
Proof. See Appendix A.

This lemma, which is an application of Bayes' rule, is reminiscent of the results in Nitzan and Paroush (1982), which characterizes the weights that a rule should attach to votes in situations where agents have heterogeneous information precision in a canonical jury setting. ${ }^{19}$

This result suggests that if a voting mechanism allows shareholders with different information precision to cast votes in proportion to the logarithm of their type, then efficiency can be reached. For instance, let us consider a group of voters with the same type space $T=\left\{e^{-10}, e^{-1}, e, e^{10}\right\}$. The voting mechanism $V=\times_{i \in N}\{-10,-1,1,10\}$ allows them to secure the efficient outcome if they each cast $\ln \left(t_{i}\right)$ votes in favor of $A$. Of course, such a mechanism also makes the "inefficient" outcome (i.e., $A$ when $\operatorname{Pr}(\alpha \mid s)<\frac{1}{2}$ and $B$ when $\left.\operatorname{Pr}(\alpha \mid s)>\frac{1}{2}\right)$ attainable, since a voter of type $t_{i}$ could cast $-\ln \left(t_{i}\right)$ votes in favor of $A$. By contrast, the mechanism $V^{\prime}=\times_{i \in N}\{-10,10\}$ does not allow the voters to reach the efficient outcome, nor the inefficient one. Hence, it is not obvious how to rank mechanisms $V$ and $V^{\prime}$ in terms of potential outcomes. As we prove in the rest of this section, when we focus on equilibrium outcomes, this indeterminacy is resolved.

\subsection{Comparison of Finite Mechanisms}

We start this section by comparing two arbitrary finite mechanisms, with one having a ballot space that is a subset of the other's ballot space. To state our results in a compact manner it is useful to pin down this possible relationship between two voting mechanisms.

Definition 6 Consider two voting mechanisms, $V$ and $V^{\prime}$, with associated rules $V(d)=X$ and $V^{\prime}(d)=X^{\prime}$. If $X_{i}^{\prime} \subseteq X_{i}$ for every shareholder $i$ and every share distribution $d$, then $V$ is said to have a richer ballot space than $V^{\prime}$.

Thus, we have that $1 S 1 \mathrm{~V}$ has a richer ballot space than $1 P 1 \mathrm{~V}$ since, independently of the share distribution, every shareholder $i$ has at least the same ballots available to her under $1 S 1 \mathrm{~V}$ as under $1 \mathrm{P} 1 \mathrm{~V}$.

We are now ready to state our first main result: ${ }^{20}$

\footnotetext{
${ }^{19}$ Azrieli (2018) further compares the benefits of weighing the votes of individuals differently instead of assigning the same weight to everybody, considering that voters engage in sincere voting.

${ }^{20} \mathrm{We}$ prove the result under Assumption 1 for clarity of exposition. The result would still hold under the milder assumption of Weak Monotone Likelihood Property. The proof can indeed
} 
Proposition 1 Under Assumption 1, if a finite mechanism $V$ has a richer ballot space than mechanism $V^{\prime}$, and there is no decisive shareholder, then $V$ dominates $V^{\prime}$.

Proof. See Appendix A.

Why does the mechanism with the richer ballot space dominate the other? First, let us consider the comparison of the best equilibria under those two mechanisms. Recall from McLennan (1998) that in a pure common value environment, a strategy profile producing the maximal ex-ante utility must be an equilibrium. ${ }^{21}$ Given that mechanism $V$ has a richer ballot profile, any outcome under mechanism $V^{\prime}$ can be reproduced under $V$ by simply replicating the strategy. Hence, there always exists an equilibrium under $V$ that produces an ex-ante utility at least as high as in the best equilibrium under $V^{\prime} .^{22}$ Notice that for this argument to hold it is not necessary that the best equilibrium of $V$ is efficient, nor that a decisive player is absent. As long as $V$ provides more ballot options to the shareholders than $V^{\prime}$, then the best equilibrium of $V$ leads to the correct outcome with at least as high a probability as the best equilibrium of $V^{\prime}$.

Second, let us consider the comparison of the worst equilibria under those two mechanisms. The proof has two main steps. In the first step, we prove the intuitive result that under any voting mechanism, a profile of monotone strategies is at least as good as a profile where all voters vote in favor of the same outcome independently of their signal. This last profile is in fact an equilibrium in undominated strategies under any voting mechanism, provided that there is no decisive shareholder (as defined above). ${ }^{23}$ The second step is more involved and consists in proving that any equilibrium is welfare-equivalent to a profile of monotone strategies. We show that if an equilibrium exhibits strategies that are not monotonic, with a shareholder $i$ casting $x_{i}$ votes when of type $t_{i}$ but a larger amount $x_{i}^{\prime}>x_{i}$ when of lower type $t_{i}^{\prime}<t_{i}$, then it must be that the difference between $x_{i}^{\prime}$ and $x_{i}$ is small enough that

be directly adapted to that case, by defining shareholders' strategies as functions defined on the (compact) signal space rather than on the type space.

${ }^{21}$ McLennan (1998) assumes that types are finite to guarantee the existence of such a utilitymaximizing profile. We prove that profiles that maximize ex-ante utility under any finite mechanism also exist in settings with infinite types, like ours.

${ }^{22} \mathrm{Ahn}$ and Oliveros (2016) use a similar argument to show the superiority of approval voting over plurality in multi-alternative elections.

${ }^{23}$ When the shares are fragmented across several shareholders, and all are expected to employ a sufficiently biased strategy towards acceptance of the proposal, then one has incentives to vote against the proposal with all one's votes for any possible signal. Hence, casting all your votes against the reform is the unique best response to certain beliefs, and thus an undominated strategy. 
it does not affect the outcome. By following this line of reasoning, the equilibrium can be shown to be equivalent to a profile of monotone strategies.

A direct implication of Proposition 1 is that $1 S 1 \mathrm{~V}$ dominates $1 P 1 \mathrm{~V}$. Given Lemma 1 , the dominance of $1 S 1 \mathrm{~V}$ over $1 P 1 \mathrm{~V}$ seems intuitive when shareholdings are positively correlated with information accuracy (especially, when $d_{i}$ s are proportional to $\left.\ln \left(t_{i}\right) \mathrm{s}\right)$. But, if one relies on this intuition to try to assess which mechanism is better when there is no correlation between information accuracy and shareholdings, one might end up with the wrong conclusion that in such cases $1 P 1 \mathrm{~V}$ should perform better than $1 S 1 \mathrm{~V}$. This would be true only if shareholders behaved in an unsophisticated manner (i.e., if they always cast the maximum allowed number of votes for the alternative they consider more likely to be correct). As we prove, and explain after Proposition 1, when the shareholders are strategic, then $1 S 1 \mathrm{~V}$ dominates $1 \mathrm{P} 1 \mathrm{~V}$ independently of whether there is a correlation between information precision and shareholdings. We can actually show, using numerical examples, that for some values of the parameters, the dominance is strict (see Appendix $\mathrm{C}$ ).

Another implication of Proposition 1 is that $1 S 1 \mathrm{~V}$ becomes more efficient when we multiply the shares that all shareholders hold (i.e., when we split stocks) ${ }^{24}$ In particular, $V^{1 S 1 V}(k \cdot d)$ weakly dominates $V^{1 S 1 V}(d)$ for any $k \in \mathbb{N}$. This result shows that, through their effect on the ballot space of shareholders, stock splits indeed increase shareholders' ability to reveal their information about the quality of management proposals through voting.

Finally, Proposition 1 also has implications for dual class capital structures in which some shares do not carry voting rights. Allowing for shares with no voting rights reduces the effective ballot space of some shareholders, limiting their ability to convey information through voting. This affects negatively information aggregation. Therefore, $1 S 1 \mathrm{~V}$ where all shares have voting rights dominates dual class systems.

\subsection{Efficiency of Continuous Mechanisms}

In this section, we consider all voting mechanisms and focus on their efficiency. We can achieve a full characterization, both of the voting mechanisms that lead to efficiency in equilibrium, and of the complete set of efficient equilibria corresponding to each such mechanism. The overall message is two-fold: (i) to be efficient, a voting mechanism must have a ballot space at least as rich as the type space, and (ii)

\footnotetext{
${ }^{24}$ Note that increasing the number of shares proportionally has no effect under $1 P 1 \mathrm{~V}$ and $1 P 1 V-D$.
} 
when votes are fully divisible, the distribution of voting rights across shareholders is irrelevant from an information aggregation standpoint.

To prove the results in this section, we need one additional assumption:

Assumption 2 For every shareholder $i \in N, T_{i}=T$ (i.e. $\delta_{i}=\delta \in(0,1)$ ) and $t_{i}$ is continuous in $s_{i}$.

This assumptions requires that all types that are possible for one shareholder are possible -but not necessarily equally likely- for any other. Even with this assumption, our model allows for arbitrarily large differences in the expected information quality of two shareholders. ${ }^{25}$

We first characterize the unique efficient equilibrium under $1 S 1 \mathrm{~V}-\mathrm{D}$ :

Proposition 2 Under Assumptions 1 and 2, for any share distribution d, the oneshare-one-vote with divisible votes mechanism, $V^{1 S 1 V-D}(d)$, admits a unique (up to admissible multiplicative and additive constants) efficient BNE, such that $\sigma_{i}^{1 S 1 V-D}\left(t_{i}\right)=$ $c \ln t_{i}+\kappa_{i}$ with $\sum_{i \in N} \kappa_{i}=0$ and $c \in\left(0, \min \left\{\frac{-d_{i}-\kappa_{i}}{\ln \delta}, \frac{-d_{i}+\kappa_{i}}{\ln \delta}\right\}\right]$ for every $i \in N$.

Proof. See Appendix A.

The equilibrium strategy implies that $A$ wins if and only if $\sum_{i \in N} c \ln \left(t_{i}\right)>$ 0 , which guarantees that the outcome is efficient. As mentioned above, we know from McLennan (1998) that a strategy that maximizes ex ante welfare must be an equilibrium. ${ }^{26}$ Uniqueness (up to a multiplicative and an additive constant) follows from the fact that any efficient BNE $\sigma$ must guarantee that $\operatorname{sgn}\left(\sum_{i \in N} \sigma_{i}\left(t_{i}\right)\right)=$ $\operatorname{sgn}\left(\sum_{i \in N} \ln \left(t_{i}\right)\right)$. Only strategies such that $\sigma_{i}\left(t_{i}\right)=c \ln t_{i}+\kappa_{i}$ with $\sum_{i \in N} \kappa_{i}=0$ and $c \in\left(0, \min \left\{\frac{-d_{i}-\kappa_{i}}{\ln \delta}, \frac{-d_{i}+\kappa_{i}}{\ln \delta}\right\}\right]$ for every $i \in N$ satisfy that condition. ${ }^{27}$ The maximum value of $c$ simply guarantees that the ballot of any given type of voter fits in the ballot space.

${ }^{25}$ The continuity of the mapping from signals to types amounts to having a connected type space. This helps us to fully focus on the essential nature of the strategies in an efficient equilibrium, without being distracted by the possibility of multiple best responses. Indeed, when gaps in the type space are allowed all the equilibria that we identify still exist, but additional ones emerge due to indifferences of types close to points of discontinuity.

${ }^{26}$ We can even show that $\sigma^{1 S 1 V-D}$ is an ex-post equilibrium: no shareholder has incentives to deviate ex post, when all types are known.

${ }^{27}$ This result highlights a challenge for shareholders: they need to coordinate on the correct multiplicative and additive constants. It seems that a focal equilibrium is the one in which shareholders use the highest possible vote weight when they receive the most informative signal and zero votes when completely indifferent. This prediction could be tested in the laboratory. 
Next, we show that continuous voting mechanisms, like $1 P 1 \mathrm{~V}-\mathrm{D}$ and $1 S 1 \mathrm{~V}-\mathrm{D}$, are the only efficient mechanisms:

Proposition 3 Under Assumptions 1 and 2, a voting mechanism implements the efficient outcome in equilibrium if and only if it is a continuous voting mechanism.

Proof. See Appendix A.

The "if" part of the proposition follows from the fact that the efficient equilibrium of the $1 S 1 \mathrm{~V}-\mathrm{D}$ mechanism can be properly rescaled to fit the ballot space of any continuous voting mechanism (i.e. to fit within any open set around any vector $\left(\psi_{i}\right)_{i \in N}$ such that $\left.\sum_{i \in N} \psi_{i}=0\right)$. The "only if" part of the proposition follows from the fact that any efficient equilibrium must be strictly increasing in the shareholder's type, and only continuous voting mechanisms satisfy that requirement. To understand why it must be strictly increasing, just consider two type profiles $t$ and $t^{\prime}$ such that (i) $t_{-i}=t_{-i}^{\prime}$, (ii) $\operatorname{Pr}(\alpha \mid t)>\operatorname{Pr}(\beta \mid t)$, and (iii) $\operatorname{Pr}\left(\alpha \mid t^{\prime}\right)<\operatorname{Pr}\left(\beta \mid t^{\prime}\right)$. It must then be that $t_{i}>t_{i}^{\prime}$. But, if $\sigma_{i}\left(t_{i}\right) \leq \sigma_{i}\left(t_{i}^{\prime}\right)$, then either the outcome for $t$ or for $t^{\prime}$ is not efficient (or both).

Proposition 3 shows that all continuous voting mechanisms are efficient. This is a broad class of mechanisms, which encompasses vastly different balances of voting power across shareholders. Two extreme cases are $1 P 1 \mathrm{~V}-D$, for which shareholders have the same number of votes independently of the number of shares they hold, and $1 S 1 \mathrm{~V}$-D, for which shareholders who hold many shares have much more voting power than other shareholders who hold few shares. The equivalence of those different mechanisms from an information aggregation standpoint highlights that vote divisibility is a crucial feature of voting mechanisms, which makes imbalances in voting power across shareholders irrelevant. This means that decisions about how to distribute voting power across shareholders can be oblivious to information aggregation issues as long as votes are made fully divisible. Note that this requires all shareholders to have at least one vote. Full divisibility of votes is thus not sufficient to make dual-class voting mechanisms efficient from an information aggregation standpoint. ${ }^{28}$

\footnotetext{
${ }^{28}$ This equilibrium characterization also provides interesting insights regarding the optimal majority threshold for the passing of a proposal (see, e.g., Maug and Rydqvist 2009). Indeed, under one-person-one-vote mechanisms or one-share-one-vote mechanisms that do not allow for vote divisibility, we know that the majority requirement maximizing the probability of implementing the correct alternative varies with the information structure (i.e. for different $F_{i} \mathrm{~s}$ ). By contrast, any mechanism with fully divisible votes is efficient, for any (super-)majority requirement (see footnote
} 
There are various specific weaknesses of finite voting mechanisms (e.g., $1 P 1 \mathrm{~V}$ and $1 S 1 \mathrm{~V}$ ) that continuous voting mechanisms solve. For instance, we know that $1 P 1 \mathrm{~V}$ aggregates information asymptotically in various sets of situations (see, e.g., Feddersen and Pesendorfer 1997, Myerson 1998, Bhattacharya 2013, and Barelli et al. 2019), but that it does not typically aggregate information well in relatively small groups (see, e.g., Feddersen and Pesendorfer 1996). By contrast, continuous voting mechanisms perform well both in large and small groups.

Another weakness of finite voting mechanisms is that shareholders need to know the information technology of all other shareholders, i.e., $F_{i}(\cdot \mid \omega)$, in order to determine their optimal strategy. If they are mistaken or have ambiguous beliefs about the information technology of others, then $1 P 1 \mathrm{~V}$ may even fail to aggregate information asymptotically. By contrast, it is clear from Proposition 2 that the equilibrium strategy for any shareholder under $1 S 1 \mathrm{~V}-\mathrm{D}$ is independent of the information technology of other shareholders. This directly implies that $1 S 1 \mathrm{~V}-\mathrm{D}$, and other continuous voting mechanisms, remains efficient even in presence of such mistakes or ambiguities.

\section{Equilibrium Analysis: Endogenous Proposal}

As we discussed in the Introduction, a key role of shareholder meetings is to provide proper incentives to managers. ${ }^{29}$ The first part of our analysis abstracts from the issue of managers' incentives with respect to the design and selection of proposals, to instead focus on the information aggregation properties of voting mechanisms. In this section, we explore the effect of the voting mechanism on the managers' incentives. In particular, we allow the management to decide whether to put a proposal (of exogenously given quality) to the vote. The management thus has veto power on the proposal. We allow for management's preferences to differ from shareholders' preferences over two dimensions. First, the manager may prefer the proposal to be adopted in both states. We then say that the manager is misaligned.

15, p.7). Hence, continuous voting mechanisms remain efficient even if a super majority threshold is necessary for alternative reasons (e.g. to prevent aggressive acquisition attempts) or is mandated by law (e.g., for changes in the Bylaws of the firm).

${ }^{29}$ The proper use of information by shareholders appears to be crucial. As mentioned in Harford et al. (2018, p. 425): "Monitoring by imperfectly informed market participants can lead managers to make myopic investment decisions (Stein, 1988). Indeed, most managers admit that they are willing to sacrifice long-term shareholder value for short-term profits (Graham et al., 2005)." Similarly, Richardson (2000) finds a positive relationship between information asymmetry, between shareholders and managers, and earnings management (i.e., funky accounting practices by managers). 
Second, the manager may incur a cost if a proposal is rejected at the shareholder meeting. As we explain in more details below, it is useful to consider separately the cases with costless rejection and the one with costly rejection. The former is indeed especially appropriate to explore how the behavior of the aligned manager is affected by the voting mechanism, whereas the latter is about the behavior of the misaligned manager.

\subsection{A General Model}

We introduce a general model that encompasses all the cases discussed in this section. The shareholders are modeled as in our baseline model. We focus on the comparison between continuous voting mechanisms (such as $1 P 1 \mathrm{~V}$-D and $1 S 1 \mathrm{~V}-\mathrm{D}$ ), that are efficient at the voting stage, and finite voting mechanisms (such as $1 P 1 \mathrm{~V}$ and $1 S 1 \mathrm{~V}$ ), that are not efficient at the voting stage. For the sake of conciseness and concreteness, we focus on two specific mechanisms: $1 P 1 \mathrm{~V}$ and $1 P 1 \mathrm{~V}-\mathrm{D}$.

We introduce a new player: the manager, denoted by $M$. She does not belong to the set of shareholders. ${ }^{30}$ After receiving a signal (more details below), the manager decides to either put the proposal to a vote $\left(x_{M}=P\right)$ or veto it $\left(x_{M}=V\right)$. If the manager vetoes, the proposal is not considered by the shareholders, and the outcome is $B$. If the manager calls for a vote, shareholders decide whether to accept it (outcome $A$ ) or reject it (outcome $B$ ).

Before making her decision, the manager receives a signal $s_{M} \in[0,1]$. In any state $\omega$, the signal $s_{M}$ is drawn from a distribution $F_{M}(\cdot \mid \omega)$, with density $f_{M}(\cdot \mid \omega)$, independently from the signals of the shareholders. We assume that the manager's type $t_{M}$ is weakly increasing in $s_{M}$. In what follows, we consider two cases: either the manager's type space $T_{M}$ is compact, i.e. $T_{M}=\left[\delta_{M}, 1 / \delta_{M}\right]$ (as for the shareholders), or the manager knows the state of nature, i.e., $T_{M}=\{0, \infty\}$.

The utility of the manager $u_{M}$ can be decomposed into two parts: an outcomeutility $u_{M}^{o}$ and a reputation cost $c$. The outcome-utility depends on whether the manager is aligned $(a=1)$ with the shareholders or misaligned $(a=0)$. When aligned, the manager has the same outcome-utility as a shareholder holding one share. In particular, for any decision $O \in\{A, B\}$ and state $\omega \in\{\alpha, \beta\}$, we have:

$$
u_{M}^{o}(O \mid \omega, a=1)=\mathbf{1}_{\{O=A, \omega=\alpha\}}-\mathbf{1}_{\{O=A, \omega=\beta\}} .
$$

\footnotetext{
${ }^{30}$ This assumption is not crucial for our results except for the case of a fully aligned manager under $1 P 1 V-D$. Without that assumption, the manager never vetoes in that case, making it less interesting.
} 
When misaligned, the manager wants the reform to pass in both states:

$$
u_{M}^{o}(O \mid \omega, a=0)=\mathbf{1}_{\{O=A\}} .
$$

We assume that the manager has an ex-ante probability $\mu \in[0,1]$ to be misaligned, and that the draw of $a$ is independent of both the state and the signals. Only the manager knows whether she is aligned.

The manager also incurs a cost $c \geq 0$ if the proposal is turned down by shareholders at the meeting. Shareholders know the value of $c$. The utility of the manager can be written as:

$$
u_{M}\left(O, x_{M} \mid \omega, a\right)=u_{M}^{o}(O \mid \omega, a)-c \times \mathbf{1}_{\left\{x_{M}=P, O=B\right\}} .
$$

While the game has now an additional player, we still use the notion of dominance defined for the exogenous proposal case: a voting mechanism dominates another if the best and worst equilibria of the former mechanism (in terms of informational efficiency, taking both shareholders' and the manager's signals into account) outperform the best and worst equilibria of the latter.

In order to analyze the endogenous proposal case, we need to consider asymmetric priors. In particular, shareholders may attach different probabilities to each state because of the strategic behavior of the manager. Crucial to the analysis that follows, our result that continuous voting mechanisms such as $1 P 1 \mathrm{~V}-D$ dominate finite voting mechanisms such as $1 P 1 \mathrm{~V}$ is robust to asymmetric priors. In fact, continuous voting mechanisms remain efficient in that case. ${ }^{31}$

\subsection{Costless Rejection}

We start by investigating the case for which the manager does not incur a cost if her proposal is turned down at the shareholders meeting $(c=0)$. The only potential source of conflict with shareholders is then whether the proposal should pass only in state $\omega=\alpha$, or in both states. This case is useful to explore the effect of voting mechanisms on the behavior of the manager when aligned. Indeed, when

\footnotetext{
${ }^{31} \mathrm{~A}$ simple modification of Lemma 1 to allow for asymmetric priors shows that the posterior $\operatorname{Pr}(\alpha \mid s)>\frac{1}{2}$ if and only if $\sum_{i \in N} \ln \left(t_{i}\right)>\ln \left(\frac{1-\operatorname{Pr}(\alpha)}{\operatorname{Pr}(\alpha)}\right)$. Thus, in order to implement the efficient decision at the voting stage, shareholders need to compensate for the different likelihood across states. Under $1 P 1 V-D$, shareholders can still implement the efficient decision with the equilibrium $\sigma_{i}^{1 P 1 V-D}\left(t_{i}\right)=c \ln t_{i}+\kappa_{i}$, with $\sum \kappa_{i}=c \ln \left(\frac{\operatorname{Pr}(\alpha)}{1-\operatorname{Pr}(\alpha)}\right)$.
} 
rejection is costless for the manager, the misaligned manager always calls for a vote, independently of the voting mechanism. We show that full divisibility of the votes remain desirable in this case (i.e., $1 P 1 \mathrm{~V}$ - $D$ dominates $1 P 1 \mathrm{~V}$ ).

\subsubsection{Comparison of Voting Mechanisms}

The following proposition shows that, when rejection of the proposal at the meeting is costless for the manager, $1 \mathrm{P} 1 \mathrm{~V}-\mathrm{D}$ continues to perform better than $1 \mathrm{P} 1 \mathrm{~V}$ :

Proposition 4 Under Assumptions 1 and 2, for every probability $\mu \in[0,1]$ that the manager is misaligned, the one-person-one-vote with divisible votes mechanism $\left(V^{1 P 1 V-D}\right)$ dominates the one-person-one-vote mechanism $\left(V^{1 P 1 V}\right)$. For some parameter values, the dominance is strict.

Proof. See Appendix B.

To understand this result, let us first suppose that the manager is aligned with shareholders with probability one (i.e., $\mu=0$ ). When an aligned manager takes the behavior of shareholders as given, she calls for a vote only if she believes that it is sufficiently likely to be a good proposal (i.e., that the state is $\alpha$ ). The definition of "sufficiently likely" depends on the magnitude of type- $I$ (outcome $A$ in state $\beta$ ) and type- $I I$ (outcome $B$ in state $\alpha$ ) errors at the voting stage. The higher the overall probability of error, the higher the manager's incentives to veto. As we have seen in the previous section, for any given information structure, errors at the voting stage are higher under $1 P 1 \mathrm{~V}$ than $1 P 1 \mathrm{~V}$-D. Hence, the aligned manager has stronger incentives to veto the proposal under $1 P 1 \mathrm{~V}$ than $1 P 1 \mathrm{~V}-D$.

Given that the manager is (partially) informed about the state of the world, her decision of whether to veto influences the beliefs of shareholders about the quality of the proposal. The higher propensity of the manager to veto under $1 \mathrm{P} 1 \mathrm{~V}$ implies that the decision to call for a vote is a stronger signal that the proposal is good under $1 P 1 V$ than $1 P 1 V-D$. Hence, shareholders start their meeting with more precise information (and more favorable to $A$ ) under $1 P 1 V$ than $1 P 1 V$ - D, which increases the probability that they make a correct decision.

There is thus a trade-off between the informational efficiency of $1 P 1 V$ - $D$ in the voting phase and the poorer selection incentives it gives to the manager before the meeting. In Proposition 4, we prove that, despite this trade-off, $1 P 1 V$ - $D$ continues to dominate $1 P 1 \mathrm{~V}$. For the case of a perfectly aligned manager, the intuition is 
similar to that of previous results: conditional on the state, all players prefer the same outcome. Since $1 P 1 V-D$ gives more flexibility to transmit information than $1 P 1 \mathrm{~V}$, it dominates.

The trade-off between selection and voting efficiency remains when the manager is misaligned with positive probability, i.e., $\mu>0$. However, it is attenuated because, under both $1 P 1 \mathrm{~V}-\mathrm{D}$ and $1 \mathrm{P} 1 \mathrm{~V}$, when the manager is misaligned $(a=0)$, she always puts the proposal to a vote. This is true even if she receives a very precise signal that the proposal is undesirable. Hence, even if shareholders take into account that the ratio of bad proposals over good ones proposed by a misaligned manager is higher than the same ratio for an aligned manager, the effect is the same under the two mechanisms. We thus have that the dominance of $1 P 1 \mathrm{~V}-\mathrm{D}$ over $1 \mathrm{P} 1 \mathrm{~V}$ is stronger the higher the probability $\mu$ that the manager is misaligned.

\subsubsection{Empirical Implications}

This analysis sheds a new light on the very high approval rate of management's proposals by shareholders in practice (see, e.g., Maug and Rydqvist 2009, Babenko et al. 2018, and Bach and Metzger 2019). It has been argued (informally) that such a high rate can be explained by the selection of proposals by managers (see, e.g., Becht et al. 2016). The idea is that the fear of having a proposal turned down, which has negative consequences for the managers, gives managers incentives to withhold low quality proposals. This means that only high quality proposals are put to a vote, and are approved at a very high rate.

In our model, shareholders approve the proposal more frequently when the manager has the power to veto it than when she does not. Yet, the mechanism at play is different. As we explained above, it does not necessarily rely on the management incurring a cost when its proposal is rejected by shareholders, as we assumed $c=0$ in this subsection. It instead relies on the manager's willingness to make decisions that are beneficial for the firm and shareholders. This is another source of selection for proposals at shareholders meetings that has implications for the empirical literature studying the effects of shareholders voting on firms' performance.

Our analysis also produces a testable prediction: the approval rate of proposals at the shareholder meeting is decreasing in $\mu$, the probability that the manager is misaligned. Different measures of alignment between shareholders and management could be used to test this prediction, such as the extent of the CEO equity-based compensation as in Datta et al. (2001), bonus-compensation as in Grinstein and 
Hribar (2004), and the sensitivity of the CEO compensation to stock performance post acquisition as in Harford and Li (2007).

\subsection{Costly Rejection}

We now analyze the case in which the manager incurs a cost when her proposal is rejected at the meeting $(c>0)$. Costly rejection moderates the incentives of the manager to call for a vote. In contrast with the costless rejection case, the misaligned manager may then choose to veto the proposal. This moderating effect is stronger (i) the higher the cost of rejection $c$, and (ii) the higher the probability of rejection by shareholders. Given that the probability of rejection is generically different under $1 P 1 \mathrm{~V}-\mathrm{D}$ and $1 P 1 \mathrm{~V}$, the moderating effect affects manager's incentives differently under the two mechanisms.

Key to the overall information aggregation performance of a voting mechanism is how it incentivizes the misaligned manager to veto the proposal when it is bad. To center the analysis on this dimension of the problem, we focus on the special case of our model in which the manager is perfectly informed about the quality of the proposal (i.e., she knows the state of the world); an assumption that also helps with tractability. As we show below, in that case, the only equilibrium choice by the manager that may vary with the voting efficiency of the mechanism is when she is misaligned and the proposal is bad.

Our main finding is that there are situations in which the moderating effect is sufficiently stronger under $1 P 1 \mathrm{~V}$ so that the better selection of proposals by the misaligned manager under $1 P 1 V$ more than compensates the higher voting efficiency of $1 P 1 \mathrm{~V}-\mathrm{D}$. Then, $1 \mathrm{P} 1 \mathrm{~V}$ dominates $1 P 1 \mathrm{~V}-\mathrm{D}$.

\subsubsection{Equilibrium Behavior}

The following Lemma stems directly from the assumption that the manager is perfectly informed, the fact that in the good state $(\omega=\alpha)$ both types of managers have the same preferences, and the fact that, under both $1 P 1 \mathrm{~V}-\mathrm{D}$ and $1 P 1 \mathrm{~V}$, the probability of rejection is higher in the bad state $(\omega=\beta)$.

Lemma 2 In equilibrium, (i) in state $\alpha$, the manager makes the same decision whether aligned or misaligned, (ii) in state $\beta$, the aligned manager always vetoes, and (iii) the misaligned manager vetoes with a (weakly) higher probability in state $\beta$ than in state $\alpha$. 
Lemma 2 narrows down the potential equilibrium strategy to three types: (i) the manager only vetoes when aligned and the state is bad; (ii) when the state is good, the manager never vetoes, and when the state is bad, the manager vetoes with positive probability $(<1)$ if misaligned and with probability 1 if aligned; (iii) the manager vetoes in both states. That latter type of equilibria, which occurs only with specific out-of-equilibrium beliefs for shareholders or very large $c$, is uninteresting. We thus focus on the two other types of equilibria in what follows.

In types of equilbria (i) and (ii), the manager never vetoes in the good state (regardless of her type), she always vetoes a bad reform when aligned, and vetoes in the bad state with probability $\gamma_{V} \in[0,1)$ when misaligned. ${ }^{32}$ Together, $\mu$ (the probability the manager is misaligned) and $\gamma_{V}$ determine the shareholders' prior that the reform is good conditional on a vote (i.e., $\left.\operatorname{Pr}\left(\alpha \mid \gamma_{V}\right)=\left(1+\mu\left(1-\gamma_{V}\right)\right)^{-1}\right)$, which in turn determines the shareholders' optimal behavior at the meeting. In equilibrium, it must be that the probability of vetoing in the bad state is optimal given the best response of shareholders.

A key driver of the manager's behavior is the probability that the proposal is rejected by shareholders. The following Lemma highlights that for the misaligned manager, the decision whether to veto the proposal in the bad state relies only on the probability that the proposal is rejected in that state, i.e., 1 minus the probability of type- $I$ error, $p_{I}\left(\gamma_{V}\right)$.

Lemma 3 In equilibrium, the probability that the misaligned manager vetoes in the bad state, $\gamma_{V}^{*}$, must be such that $p_{I}\left(\gamma_{V}^{*}\right) \geq \frac{c}{1+c}$. When $p_{I}(0)>\frac{c}{1+c}$, the equilibrium is such that the manager never vetoes, i.e., $\gamma_{V}^{*}=0$. When $p_{I}(0)<\frac{c}{1+c}$, the equilibrium is such that the misaligned manager vetoes in the bad state with positive probability, i.e. $\gamma_{V}^{*} \in(0,1)$, with $p_{I}\left(\gamma_{V}^{*}\right)=\frac{c}{1+c}$.

Proof. See Appendix B.

To understand the behavior of the manager under the different mechanisms, we thus need to understand how the probability of type- $I$ error, $p_{I}$, varies across voting mechanisms. The misaligned manager has stronger incentives to veto the proposal if the probability of type- $I$ error is low. The issue is that $1 P 1 \mathrm{~V}-D$ and $1 P 1 \mathrm{~V}$ cannot be neatly ranked based on that probability of error: depending on the situation, it

\footnotetext{
${ }^{32}$ Note that if $\gamma_{V}=1$, the shareholders can make the inference that conditional on voting, the state must be good. In that case, the incentives of shareholders is to always approve the reform. But this would give the manager incentives to deviate from $\gamma_{V}=1$. Hence, this cannot be an equilibrium.
} 


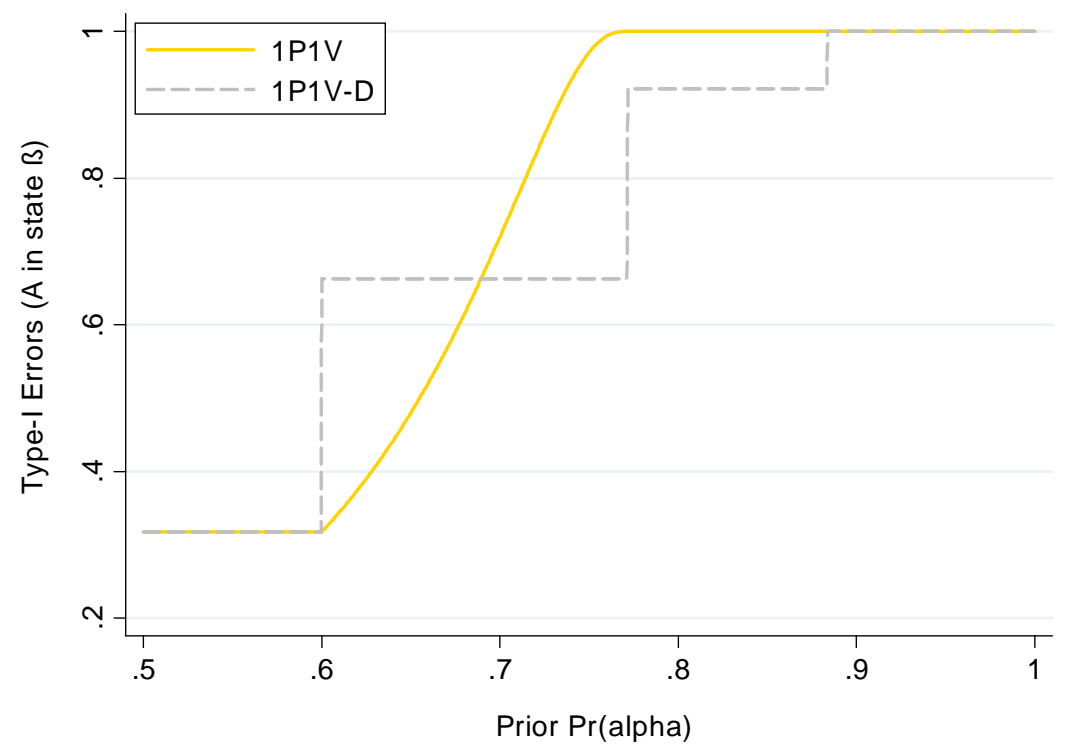

Figure 1: Probability of a type-I error under $1 \mathrm{P} 1 \mathrm{~V}$ and $1 \mathrm{P} 1 \mathrm{~V}-\mathrm{D}$ as a function of the prior on state $\alpha$ (with an exogenous agenda). Parameters assumed: $n=5$, and binary signals with $\operatorname{Pr}\left(s_{\alpha} \mid \alpha\right)=\operatorname{Pr}\left(s_{\beta} \mid \beta\right)=0.6$.

may be higher or lower under $1 P 1 \mathrm{~V}$-D than $1 P 1 \mathrm{~V}$. This is illustrated in Figure 1, which shows the probability of type- $I$ errors as a function of the prior for the case of an exogenous proposal. This prior is relevant because, as explained above, it increases with $\gamma_{V}$, the probability that the manager vetoes the proposal in the bad state. The figure thus implicitly shows that for some values of $\gamma_{V}$, the probability of type- $I$ error is higher under $1 P 1 \mathrm{~V}-\mathrm{D}$ and for other values it is higher under $1 P 1 \mathrm{~V}$. Whether the misaligned manager ends up vetoing more under one mechanism or the other in equilibrium thus depends on the specifics of the situations. ${ }^{33}$

\subsubsection{Comparison of Mechanisms}

We are now in position to compare continuous and finite voting mechanisms. ${ }^{34}$ First, we can show that there is always a cost of rejection $c$ sufficiently small so that $1 P 1 \mathrm{~V}$ -

\footnotetext{
${ }^{33}$ In the case of binary signals, the optimal decision is simply implemented with a threshold: the reform is only accepted given a number of signals in favor of the reform. All priors that have the same optimal threshold generate the same probability of type-I error. This, together with the fact that the optimal threshold decreases with the prior explains the step function under $1 P 1 \mathrm{~V}$ - $D$ in Figure 1.

${ }^{34}$ In this subsection, we focus on the optimal symmetric equilibria at the voting stage.
} 
$D$ continues to dominate $1 P 1 V$.

Proposition 5 Under Assumptions 1 and 2, when the manager is perfectly informed, $\exists \bar{c}>0$ such that, if $c<\bar{c}$, the one-person-one-vote with fully divisible votes mechanism $\left(V^{1 P 1 V-D}\right)$ dominates the one-person-one-vote mechanism $\left(V^{1 P 1 V}\right)$.

Proof. See Appendix B.

The intuition is as follows. As we have seen above, the incentives to veto of the misaligned manager depends the cost of rejection $c$ and the probability of type- $I$ error. When $c$ is sufficiently small, the incentives to veto of the misaligned manager are very weak under both $1 P 1 \mathrm{~V}-\mathrm{D}$ and $1 P 1 \mathrm{~V}$ and she never vetoes. The comparison of the two mechanisms then depends exclusively on their voting efficiency. And we know from the results in the previous section that $1 P 1 V$ - $D$ has a higher voting efficiency.

For larger cost of rejection $c$, the behavior of the misaligned manager in the bad state generally differs under the two mechanisms. However, the effect can either reinforce or compensate the higher voting efficiency of $1 P 1 \mathrm{~V}-\mathrm{D}$ (which is defined in terms of both types of errors). When the probability of type- $I$ error is lower under $1 P 1 V-D$ in equilibrium, the manager vetoes more often in the bad state under $1 P 1 \mathrm{~V}$-D. The better selection of proposals by the manager then reinforces the voting efficiency advantage of $1 P 1 V-D$. By contrast, when the probability of type- $I$ error is lower under $1 P 1 V$ in equilibrium, the manager vetoes more often in the bad state under $1 P 1 \mathrm{~V}$. The better selection of proposals by the manager under $1 P 1 \mathrm{~V}$ then compensates for its lower voting efficiency. As we show in the following proposition, in equilibrium, the selection effect can be strong enough to overturn the higher voting efficiency of $1 P 1 V-D$.

Proposition 6 Under Assumptions 1 and 2, when the manager is perfectly informed, the one-person-one-vote with fully divisible votes mechanism $\left(V^{1 P 1 V-D}\right)$ does not necessarily dominate the one-person-one-vote mechanism ( $\left.V^{1 P 1 V}\right)$. For some parameter values, in the best symmetric equilibrium, shareholders are strictly better off under $V^{1 P 1 V}$ than $V^{1 P 1 V-D}$.

The proof of this result relies on a numerical example. Let us consider the following case: there are 5 shareholders $(n=5)$, the prior of the good state is $\operatorname{Pr}(\alpha)=0.5$, and there are binary signals with $\operatorname{Pr}\left(s_{\alpha} \mid \alpha\right)=\operatorname{Pr}\left(s_{\beta} \mid \beta\right)=0.6$. Suppose, moreover, that the manager is misaligned with probability $\mu=0.6$, and that the 


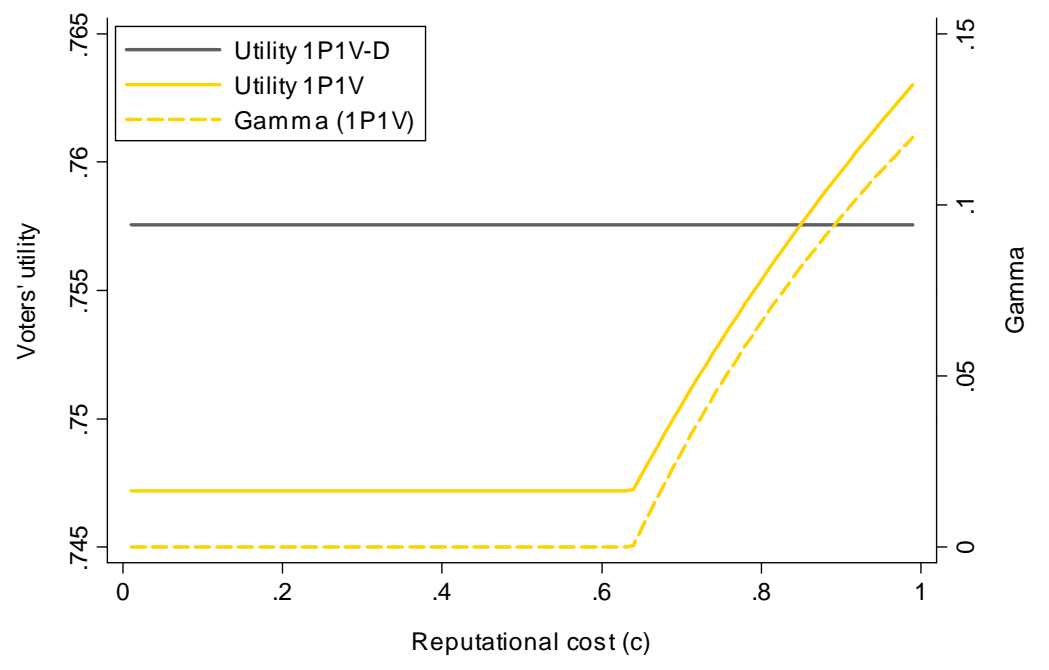

Figure 2: Welfare comparison between 1P1V-D and 1P1V. The parameters assumed for this comparison are $n=5, \operatorname{Pr}(\alpha)=0.5$, and binary signals with $\operatorname{Pr}\left(s_{\alpha} \mid \alpha\right)=$ $\operatorname{Pr}\left(s_{\beta} \mid \beta\right)=0.6$.

cost of rejection $c \in[0,1]$. Figure 2 shows how the utility of shareholders varies with $c$. For sufficiently low values of $c$ (below 0.63 ), when the manager calls for a vote with probability 1 in the bad state under both mechanisms, then $p_{I}^{1 P 1 V-D}(0)$, $p_{I}^{1 P 1 V}(0)>\frac{c}{1+c}$. Hence, it is a best response for the manager not to veto, i.e. $\gamma_{V}^{*}=0$, under both $1 P 1 \mathrm{~V}-\mathrm{D}$ and $1 \mathrm{P} 1 \mathrm{~V}$. Since there is no differential selection, the only difference across mechanisms comes from their ability to aggregate the information dispersed among shareholders. Consistent with what we have seen in the case of an exogenous proposal, $1 P 1 \mathrm{~V}-\mathrm{D}$ then dominates $1 \mathrm{P} 1 \mathrm{~V}$. This illustrates the result in Proposition 5. By contrast, when $c$ is higher than 0.63 , the same strategy by the manager implies $p_{I}^{1 P 1 V-D}(0)>\frac{c}{1+c}>p_{I}^{1 P 1 V}(0)$. It is thus not a best response for the manager to always call for a vote under $1 P 1 \mathrm{~V}$ (but it is under $1 P 1 \mathrm{~V}-\mathrm{D}$ ). In equilibrium, she vetoes the proposal in the bad state with probability $\gamma_{V}^{*} \in(0,1)$ under $1 P 1 \mathrm{~V}$, with $\gamma_{V}^{*}$ increasing in $c$. This selection of proposal by the manager is beneficial to shareholders. For $c$ sufficiently large (above 0.85 ), $1 P 1 V$ dominates $1 P 1 V-D$.

Expanding on the previous example, we can compare the utility of shareholders under $1 P 1 \mathrm{~V}-\mathrm{D}$ and $1 \mathrm{P} 1 \mathrm{~V}$ for various values of $c$ and $\mu$. Figure 3 shows that $1 \mathrm{P} 1 \mathrm{~V}$ dominates $1 P 1 V$-D when both $c$ and $\mu$ are sufficiently high. The high value of $c$ guarantees that the manager vetoes in some situations, and hence that the stronger 


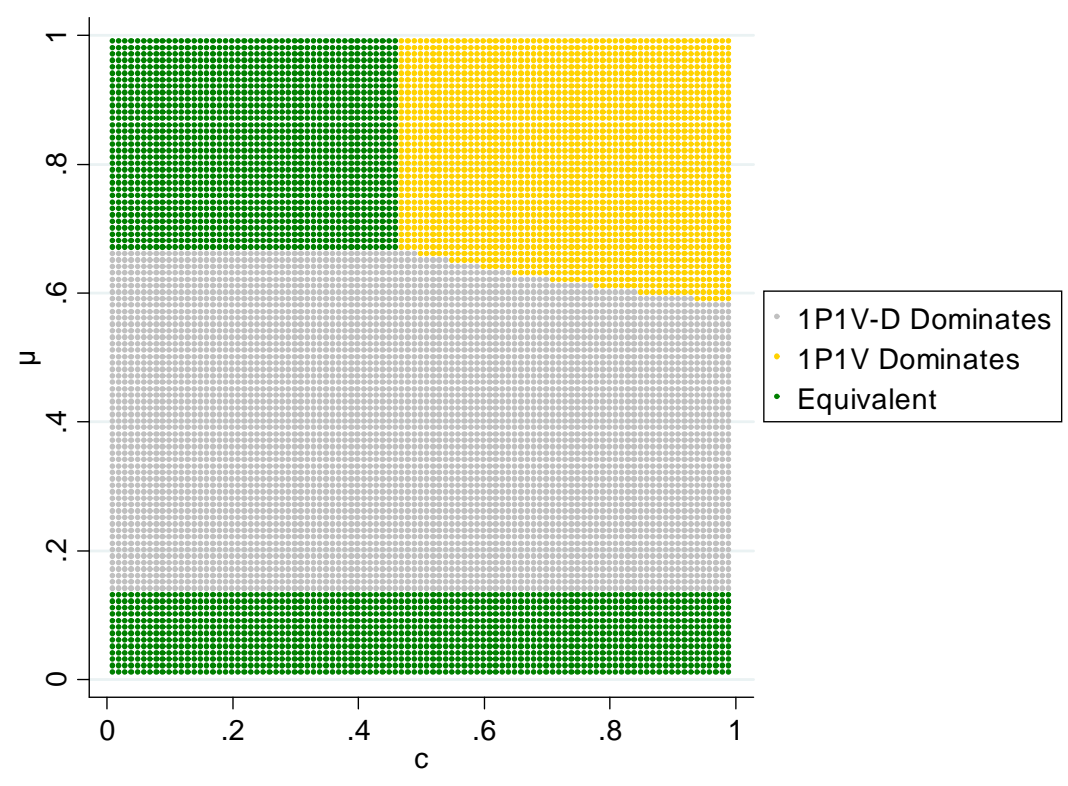

Figure 3: Welfare comparison across systems for different combinations of $c$ (horizontal axes) and $\mu$ (vertical axes). The parameters assumed for this comparison are $n=5, \operatorname{Pr}(\alpha)=0.5$, and binary signals with $\operatorname{Pr}\left(s_{\alpha} \mid \alpha\right)=\operatorname{Pr}\left(s_{\beta} \mid \beta\right)=0.6$.

selection advantage of $1 P 1 \mathrm{~V}$ is present. The high value of $\mu$ guarantees that the manager is often misaligned, and hence that the selection advantage of $1 P 1 \mathrm{~V}$ is large enough. ${ }^{35}$

\subsubsection{Empirical Implications}

In equilibrium, the quality of the proposal conditional on a vote being called is increasing in $c$ under both $1 P 1 \mathrm{~V}-D$ and $1 P 1 \mathrm{~V}$. Thus, the probability of approval of the proposal is also increasing in $c$. This confirms the informal argument in the literature (see discussion above) that the presence of a reputation cost for managers helps explain the high approval rate of the management's proposals. This result

\footnotetext{
${ }^{35}$ When aligned, the manager vetoes for sure when the state is bad. She does so under both $1 P 1 \mathrm{~V}$-D and $1 P 1 \mathrm{~V}$. When the state is good she has similar incentives as the misaligned manager (that is, she trades off the risk of rejection with the gain in case the proposal is adopted). Thus, the decision to put the proposal to a vote has a different effect on shareholders' beliefs than when the manager is always misaligned. In particular, due to the veto by the aligned manager in the bad state, the expected quality of the proposal conditional on a vote being called increases. This is beneficial for shareholders. Given that this positive selection effect when the manager is aligned occurs both under $1 P 1 \mathrm{~V}-D$ and $1 P 1 \mathrm{~V}$, it results in a decrease of the overall advantage of $1 P 1 \mathrm{~V}$ in terms of selection (this advantage only materializes when the manager is misaligned).
} 
also suggests an explanation for the much higher approval rate of management's proposals than shareholders' proposals in practice (Bach and Metzger 2019): shareholders do not suffer (as high) reputation cost when their proposals are turned down at the meeting. Differences in this cost among shareholders could potentially help explain why the approval rate of shareholder proposals is strongly associated with the identity of the sponsor (Gillan and Starks 2000).

Note also that the aforementioned testable prediction that the approval rate of managers' proposals at the meeting is decreasing in the probability that the manager is misaligned $(\mu)$, also holds when $c>0$.

\section{Discussion of Modeling Assumptions}

\subsection{State-Contingent Preferences}

A central assumption of our baseline model is that shareholders have state-contingent preferences: conditional on the state of the world, they all agree whether the management's proposal should be approved or rejected. This is a standard assumption in the literature on shareholders voting (see, e.g., Maug and Yilmaz 2002, Marquez and Yilmaz 2008, Levit and Malenko 2011, Eso, Hansen, and White 2015, Malenko and Malenko 2019, Bar-Isaac and Shapiro 2020, Meirowitz and Pi 2020, Ma and Xiong 2020). It indeed seems natural to assume that (most) shareholders share the common goal of maximizing the value of the firm.

There are various pieces of empirical evidence that are coherent with the statecontingent preferences assumption. More precisely, the literature uncovers facts that are in line with models of strategic voting making that assumption, similar to the one developed above. For instance, Maug and Rydqvist (2009) structurally estimate such a model of strategic voting using data about U.S. shareholders meetings between 1994 and 2003. They find that the voting behavior of shareholders at those meetings is in line with their model. As predicted: (i) shareholders vote more in favor of proposals when the supermajority threshold increases, and (ii) there is essentially no effect of supermajority thresholds on the acceptance rate.

Christoffersen et al. (2007) study vote trading in the US and the UK and find patterns that are in line with information aggregation theory of voting (see, e.g., Eso, Hansen, and White 2015). They indeed uncover an active market for votes, both in the US and the UK, where the average vote sells for a price of zero. Moreover, as predicted by the theory, vote trading increases (i) with asymmetric information 
among shareholders, (ii) the importance of the proposal at stake (proxied by poor performance of firm), and (iii) if the pivot probability is high. Finally, warnings of votes that violate corporate governance standards (which they interpret as a negative public signal about the proposal that reduces information asymmetry among shareholders) reduce vote trading.

Calluzzo and Dudley (2019) study the influence of proxy advisors on firm voting outcomes, policies and values. They find that, as predicted by Malenko and Malenko (2019) based on a model including shareholders with state-contingent preferences, proxy advisors have a large influence when shareholders have weak incentives to acquire information.

There is also evidence that, at first sight, appears to contradict the predictions of a model of strategic voting including shareholders with state-contingent preferences: Li et al. (2021) find that there is substantial trading by mutual funds after shareholder meetings. Yet, Meirowitz and Pi (2020) show that this is actually consistent with such a model once one takes into account that shareholders who vote in the meetings are also traders after the meeting. In such a setting, shareholders do not fully reveal information through their vote, which prevents information aggregation. This creates opportunities to trade after shareholder meetings. In our model, under $1 P 1 \mathrm{~V}$ or $1 S 1 \mathrm{~V}$, there would be a different reason for trading after the vote. Depending on the precision of their signal, shareholders have different beliefs about the probability that the decision at the meeting was correct. Hence, shareholders with sufficiently precise signals against the decision made at the meeting would be willing to sell their shares, and those with sufficiently precise signals aligned with the decision made at the meeting would be willing to buy more shares. These trading patterns are in line with the findings of $\mathrm{Li}$ et al. (2021) about the behavior of mutual funds after the meetings. ${ }^{36}$

Last but not least, it is important to stress that we are not trying to argue that every single shareholder has state-contingent preferences. Indeed, as we discuss in Appendix D, there is evidence suggesting that disagreement among shareholders may not only stem from information asymmetries. This is exactly the reason why, in that Appendix, we consider an extension of our model that allows for the presence of partisan shareholders. And we show that, in the presence of such shareholders, continuous voting mechanisms such as $1 S 1 \mathrm{~V}$-D still outperforms other voting mechanisms in terms of information aggregation.

\footnotetext{
${ }^{36}$ Our model also predicts that the trading patterns would be systematically different under $1 S 1 \mathrm{~V}$-D and $1 P 1 \mathrm{~V}$. Under $1 S 1 \mathrm{~V}$ - $D$, when voting fully aggregates information, shareholders' posteriors are identical. There is then no room for trade after the meeting.
} 


\subsection{Information Asymmetry}

Another key assumption of our model is that some shareholders are better informed than others. We view this assumption as uncontroversial. First, as explained in Knyazeva, Knyazeva, and Kostovetsky (2018, p. 681): "the precision of a trader's [...] private information may be a function of the trader's overall or company specific investment experience, local knowledge, or the extent of resources that the trader can allocate to information gathering." And, indeed, Kim and Verrecchia (1991) show that traders react differently to release of public information about a given firm, i.e., less informed traders, who revise the beliefs more, react more. Also, Iliev and Lowry (2015) and Iliev, Kalodimo, and Lowry (2018) find that mutual funds vary greatly in their reliance on proxy advisory recommendations, with the more informed voting less in line with the recommendations.

Second, the literature provides evidence that shareholders have different incentives to invest in acquisition of information (see, e.g., Chen, Harford, and Kai 2007, and Fich, Harford and Tran 2015).

Third, information asymmetries among shareholders help explain phenomena that are difficult to explain without such asymmetries (see, e.g., Glosten and Milgrom 1985 discussion of the bid-ask spread).

Finally, there is an empirical literature studying information asymmetry among shareholders, using different measures (see, e.g., Brown and Han 1992, Healy, Palep, and Sweeney 1995, Welker 1995, Iliev and Lowry 2014, and Knyazeva, Knyazeva, and Kostovetsky 2018). It points toward substantial information asymmetries among shareholders/investors. This is true both across types of shareholders (see, e.g., Sias, Starks, and Titman (2006) for evidence of the informational advantage of institutional investors over other types of investors), and within a given type (see, e.g., Knyazeva, Knyazeva, and Kostovetsky (2018) for evidence of heterogeneity among institutional investors).

\subsection{No Communication}

In our baseline model, we assume that shareholders cannot communicate before the vote. This is not an innocuous assumption. If costless, communication can indeed improve information aggregation (see, e.g., Coughlan 2001), and mute differences between voting mechanisms (see, e.g., Gerardi and Yariv 2007). The idea is simple: when shareholders have state-contingent preferences, they have incentives to truthfully reveal their private information to one another, and then vote unanimously for 
the efficient outcome.

There are nonetheless several hurdles to communication among shareholders. First, in the presence of partisan shareholders, communication is impeded (Coughlan 2001). The problem is that those shareholders have incentives to pretend that they have state-contingent preferences but that they have received a signal in favor of their preferred alternatives. And, as we show in Appendix D, continuous voting mechanisms such as $1 S 1 \mathrm{~V}$-D still dominate other voting mechanisms in the presence of such shareholders (without communication).

Second, even if there are no partisan shareholders, communication among shareholders is far from costless. In the case of most public firms, shares are distributed among many, scattered, individuals and institutions. It is thus logistically challenging to organize communication. Moreover, as explained in Malenko and Malenko (2019, p.2470), "[...] investors fear that communication with others can be considered "forming a group" [...]," which would trigger costly administrative filling requirements and, in some cases, a poison pill. There could also be a cost of publicly disclosing your information: "[...] investors are often reluctant to publicly disclose their intention to vote against management, fearing that doing so would be viewed as an activist campaign and lead to managerial retaliation."

\subsection{Vote Trading}

Our baseline model does not allow shareholders to trade votes before the meeting. Yet, we know that there is an active market for votes (Christoffersen et al. 2007) and that vote trading can be beneficial for information aggregation. Eso, Hansen, and White (2015) study vote trading and, assuming one share per shareholder, prove the existence of an efficient equilibrium under $1 P 1 \mathrm{~V}$ in which vote trades at a price of zero. In that equilibrium, uninformed shareholders sell their votes to informed shareholders. As in the case of communication, allowing for vote trading could then mute differences between continuous voting mechanisms such as $1 S 1 \mathrm{~V}$-D and other voting mechanisms. But, there are reasons to believe this is not the case.

First, note that vote trading is irrelevant under continuous voting mechanisms such as $1 S 1 \mathrm{~V}$-D: there is no gain from trade because the equilibrium is efficient. Second, there are various hurdles to vote trading under other voting mechanisms. For instance, the efficient equilibrium in Eso, Hansen, and White (2015) is not

robust to the presence of sufficiently many partisan shareholders. Moreover, we conjecture that differences in signal precision would also prevent efficient aggregation 
of information. The problem in that case is that shareholders need to know how precise their information is compared to that of other shareholders in order to decide optimally whether to "buy" or "sell" votes. There is no clear way for shareholders to do so. This issue becomes even worse if there is ambiguity about the information technology of other shareholders.

\subsection{Share Trading}

Our baseline model does not allow shareholders to trade votes before or after the meeting. A general treatment of this question is out of the scope of this paper (for recent contributions on the topic of share trading and voting, see, e.g., Bar-Isaac and Shapiro 2020, Meirowitz and Pi 2020, and Levit, Malenko, and Maug 2021a,b). However, it would be erroneous to believe that our results are not robust to some forms of share trading before or after the meeting. For instance, we could consider a pre-meeting trading model similar to Bar-Isaac and Shapiro (2020)'s. They assume that a shareholder "trades so as to maximize the overall expected value of the firm." Due to efficiency of continuous voting mechanisms, the overall expected value of the firm is maximized without trading. Shareholders would thus not have any incentive to trade shares among them before the meeting. For voting mechanisms that are not efficient, shareholders might have incentives to trade. But even if shares are fully divisible, it would not be always possible to reach the efficient outcome through share trading. What prevents efficiency is private information about the precision of the signal of the blockholder.

To see this, let us consider a case in which there is a unique blockholder holding multiple shares and all other shareholders hold one share each. Before the meeting, the blockholder can post a price and sell some of her shares to individuals who are currently not owning any fraction of the firm and are completely uninformed about the issue at hand, or to other shareholders. Other shareholders are characterized by an identical precision of information. We focus on the properties of a version of $1 S 1 \mathrm{~V}$ that compels shareholders either to use all their votes or to fully abstain. We also focus on a case in which the signal precision of the blockholder is not too high so that she would never want to buy shares in equilibrium.

If the blockholder's signal precision is publicly known, then trade (i.e., the blockholder sells some of her shares) would result in an optimal distribution of votes and

hence in an efficient outcome. In that case the price of a share simply reflects the probability that the firm makes the correct choice. Note, however, that, as discussed 
in Bar-Isaac and Shapiro (2020), there may be hurdles to share trading that could prevent the efficient outcome to materialize even when signal precision is publicly known.

If the blockholder's signal precision is her private information (consider, for instance, that her precision is identical to that of the other shareholders with some probability and otherwise higher), then there cannot be a separating equilibrium with prices corresponding to the two potential eventualities (i.e. a higher price when the blockholder's precision is high and a lower price when her information precision is low). If it were the case, then the low precision blockholder would pretend to have high precision signal in order to sell her shares at a higher price. The equilibrium price is then too low: the blockholder does not sell enough shares, and hence casts too many votes from an information aggregation standpoint. This leads to an inefficient outcome under that version of $1 S 1 \mathrm{~V}^{37}$ This implies that divisibility of votes may remain a useful tool for information aggregation when trading of shares is allowed before the meeting.

\section{Conclusions}

Shareholders typically hold different number of shares. This fact, which has been overlooked by most of the literature on voting at those meetings, raises questions about which voting mechanism should be used. In this paper, we have explored this question with a special focus on the informational efficiency of different voting mechanisms. We first considered the case in which the management is passive and does not select the proposal being voted on. We proved two main results. First, for any distribution of shareholdings, a mechanism with a richer ballot space dominates a mechanism with a poorer one, independently of whether information accuracies and shareholdings are correlated. Second, continuous voting mechanisms such as $1 S 1 V-D$ and $1 P 1 V-D$ are efficient. This second result implies that decisions about how to distribute voting power across shareholders can be oblivious to information aggregation issues as long as votes are made fully divisible.

We then considered the case in which the management decides whether to put the proposal to a vote. We uncovered a trade-off between selection and voting efficiency underlying the comparison of continuous voting mechanisms and finite

\footnotetext{
${ }^{37}$ The formal arguments backing this claim are similar to the ones employed by Bar-Isaac and Shapiro (2020) when they consider trade with endogenous information acquisition, and are therefore omitted.
} 
voting mechanisms: the higher voting efficiency of continuous voting mechanisms implies worse selection incentives for the management. We found that the negative effect of worse selection incentives on shareholders' welfare can be large enough to wash out the higher voting efficiency of continuous voting mechanisms. Then, finite voting mechanisms are better for shareholders. This suggests a possible explanation for why corporations do not make votes fully divisible in practice.

Our results also have implications for the consequences, and hence desirability, of dual class capital structures and stock buybacks and splits. Through their effect on the number of voting shares held by shareholders, dual class shares, stock buybacks and splits indeed affect shareholders' ability to reveal their information about the quality of management proposals through voting. Hence, preferential shares and stock buybacks should affect negatively the efficiency of decisions at shareholder meetings, while stock splits should affect it positively. Through their influence on voting efficiency, they should also affect the incentives of managers to select proposals that benefit shareholders. These effects complement the common arguments in favor and against dual class capital structures, stock buybacks and splits. 


\section{References}

[1] Adams, R. and D. Ferreira (2008). One Share, One Vote: The Empirical Evidence, Review of Finance, 12: 51-91.

[2] Aggarwal, R., S. Dahiya, and N. Prabhala (2019). The Power of Shareholder Votes: Evidence from Uncontested Director Elections, Journal of Financial Economics, 133: 134-153.

[3] Ahn, David S., and Santiago Oliveros (2016). Approval voting and scoring rules with common values, Journal of Economic Theory, 166: 304-310.

[4] Alissa W. 2015. Boards' Response to Shareholders' Dissatisfaction: The Case of Shareholders' Say on Pay in the UK, Europen Accounting Review, 24(4): $727-52$.

[5] Andrade, G., M. Mitchell, and E. Stafford (2001). New Evidence and Perspectives on Mergers. Journal of Economic Perspectives, 15: 103-20.

[6] Appel, I., T. Gormley, and D. Keim (2016). Passive Investors, Not Passive Owners. Journal of Financial Economics, 121: 111-141.

[7] Austen-Smith, D. and J. Banks (1996). Information Aggregation, Rationality, and the Condorcet Jury Theorem, American Political Science Review, 90: 3445 .

[8] Azrieli, Y. (2018). The price of 'one person, one vote'. Social Choice and Welfare, 50(2):353-385.

[9] Babenko, I., G. Choi, and R. Sen (2019). Management (of) Proposals. mimeo.

[10] Bach, L. and D. Metzger (2019). How Close Are Shareholder Votes? Review of Financial Studies, 32(8): 3183-3214.

[11] Barelli, P., S. Bhattacharya, and L. Siga (2019). Full Information Equivalence in Large Elections. mimeo.

[12] Bar-Isaac, H. and J. Shapiro (2020). Blockholder Voting. Journal of Financial Economics, 136 (3): 695-717.

[13] Becht, M., A. Polo, and S. Rossi (2017). Does Mandatory Shareholder Voting Prevent Bad Acquisitions?, Review of Financial Studies, 29(11): 3035-67. 
[14] Bhattacharya, S. (2013). Preference Monotonicity and Information Aggregation in Elections, Econometrica, 81(3), 1229-1247.

[15] Bolton, P., Li, T., Ravina, E., \& Rosenthal, H. (2020). Investor ideology, Journal of Financial Economics, 137 (2): 320-352.

[16] Bond, P. and H. Eraslan (2010). Strategic Voting over Strategic Proposals, Review of Economic Studies, 77(2): 459-490.

[17] Bouton, L., and M. Castanheira (2012). One Person, Many Votes: Divided Majority and Information Aggregation, Econometrica, 80(1): 43-87.

[18] Bouton, L., A. Llorente-Saguer, and F. Malherbe (2018). Get Rid of Unanimity: The Superiority of Majority Rules with Veto Power, Journal of Political Economy, 126(1): 107-149.

[19] Bouwman, C. H. S., K. Fuller, and A. S. Nain (2009). Market Valuation and Acquisition Quality: Empirical Evidence. Review of Financial Studies, 22: 63379.

[20] Brav, A., D. Cain, and J. Zytnick (2019). Retail Shareholder Participation in the Proxy Process: Monitoring, Engagement, and Voting, ECGI Working Paper Series in Finance, 637/2019.

[21] Brown, L.D. and J. Han (1992). The Impact of Annual Earnings Announcements on Convergence of Beliefs. Accounting Review, 67: 862-875.

[22] Burkart, M. and S. Lee (2008). One Share-One Vote: the Theory, Review of Finance, 12: 1-49.

[23] Cai, J., J. Garner, and R. Walking (2009). Electing Directors, Journal of Finance, 64(5): 2389-2421

[24] Calluzzo, P. and E. Dudley (2019). The Real Effects of Proxy Advisors on the Firm. Financial Management, 48: 917-943.

[25] Chen, Xia, Harford, Jarrad, Li, Kai (2007). Monitoring: which institutions matter? Journal of Financial Economics, 86, 279-305.

[26] Chevalier, Judith, and Glenn Ellison (1999). Career concerns of mutual fund managers, Quarterly Journal of Economics, 114, 389-432. 
[27] Christoffersen, S. E., Geczy, C. C., Musto, D. K. and Reed, A. V. (2007), Vote Trading and Information Aggregation. Journal of Finance, 62: 2897-2929

[28] Cohen, Lauren, and Breno Schmidt (2009). Attracting flows by attracting big clients, Journal of Finance, 64, 2125-2151.

[29] Conyon M. and G. Sadler (2010). Shareholder Voting and Directors' Remuneration Report Legislation: Say on Pay in the UK. Corporate Governance: An International Review, 18: 296-312.

[30] Coughlan, P. (2001). In Defense of Unanimous Jury Verdicts: Mistrials, Communication and Strategic Voting. American Political Science Review, 94: 37593.

[31] Cunat, V., M. Gine, and M. Guadalupe (2016). Say Pays! Shareholder Voice and Firm Performance. Review of Finance, 1799-1834.

[32] Cvijanovic, D., Dasgupta, A., and Zachariadis, K.E. (2016). Ties that Bind: How Business Connections Affect Mutual Fund Activism, Journal of Finance, 71(6): 2933-2966.

[33] Cvijanovic, D., Dasgupta, A., and Zachariadis, K.E. (2019). Free-riders and Underdogs: Participation in Corporate Voting, mimeo.

[34] Datta, Sudip, Mai Iskandar-Datta, and Kartik Raman (2001). Executive Compensation and Corporate Acquisition Decisions, Journal of Finance, 56: 22992336.

[35] Davis, Gerald F., and E. Han Kim (2007). Business ties and proxy voting by mutual funds, Journal of Financial Economics, 85, 552-570.

[36] Edmans, A., and C. Holderness (2017). Blockholders: A Survey of Theory and Evidence, Handbook of the Economics of Corporate Governance, Volume 1.

[37] Eso, P., S. Hansen, L. White (2015). A Theory of Vote Trading and Information Aggregation. mimeo.

[38] Feddersen, T. and W. Pesendorfer (1996). The Swing Voter's Curse, American Economic Review, 86: 408-424.

[39] Feddersen, T. and W. Pesendorfer (1997). Voting Behavior and Information Aggregation in Elections with Private Information, Econometrica, 65: 10291058. 
[40] Feddersen, T. and W. Pesendorfer (1998). Convicting the Innocent: the Inferiority of Unanimous Jury Verdicts, American Political Science Review, 92: 23-35.

[41] Ferri, F. and D. Maber, Say on Pay Votes and CEO Compensation: Evidence from the UK, Review of Finance, 17(2): 527-563.

[42] Fich, E., Harford, J., and A. Tran (2015). Motivated Monitors: The Importance of Institutional Investors' Portfolio Weights. Journal of Financial Economics, 118: $21-48$.

[43] Fos, V., K. Li, M. Tsoutsoura (2018). Do Director Elections Matter?, Review of Financial Studies, 31(4): 1499-1531

[44] Frankenreiter, J., C. Hwang, Y. Nili, and E. Talley (2021). Cleaning Corporate Governance. University of Penn Law Review, forthcoming.

[45] Fu, F., L. Lin, and M. Officer (2013). Acquisitions Driven by Stock Overvaluation: Are They Good Deals? Journal of Financial Economics, 109: 24-39.

[46] Gantchev, N. and M. Giannetti (2020). The Costs and Benefits of Shareholder Democracy: Gadflies and Low-Cost Activism. ECGI Finance Working Paper, $586 / 2018$.

[47] Gerardi, D. and L. Yariv (2007). Deliberative Voting. Journal of Economic Theory, 114: 357-394.

[48] Gershkov, A. and B. Szentes (2009). Optimal Voting Schemes with Costly Information Acquisition. Journal of Economic Theory, 144(1): 36-68.

[49] Gillan, S. and L. Starks (2000). Corporate Governance Proposals and Shareholder Activism: The Role of Institutional Investors, Journal of Financial Economics, 57: 275-305.

[50] Glosten, L. and P. Milgrom (1985). Bid, Ask and Transaction Prices in a Specialist Market with Heterogeneously Informed Traders. Journal of Financial Economics, 14(1): 71-100.

[51] Gompers, P., J. Ishii, and A. Metrick (2003). Corporate Governance and Equity Prices. Quarterly Journal of Economics, 118(1): 107-55. 
[52] Gray, S., T. Smith, and R. Whaley (2003). Stock Splits: Implications for Investor Trading Costs. Journal of Empirical Finance, 10: 271-303.

[53] Grinstein, Y. and P. Hribar (2004). CEO Compensation and Incentives: Evidence from M\&A Bonuses. Journal of Financial Economics, 73: 119-143.

[54] Hansmann, H. and Pargendler, M., 2013. The evolution of shareholder voting rights: Separation of ownership and consumption. Yale Law Journal, 123, p.948.

[55] Harford, J., M. Humphery-Jenner, and R. Powell. 2012. The Sources of Value Destruction in Acquisitions by Entrenched Managers. Journal of Financial Economics, 106: 247-61.

[56] Harford, J., Kecskes, A., and M. Mansi (2018). Do long-term investors improve corporate decision making? (Working Paper).

[57] Harford, J., and K. Li (2007). Decoupling CEO Wealth and Firm Performance: The Case of Acquiring CEOs. Journal of Finance, LXII(2): 917-949.

[58] Hayden, G. and M. Bodie (2008). The False Promise of One Share, One Vote. Legal Studies Research Paper Series, 08-01.

[59] Healy, P., K. Palepu, and A. Sweeney (1995). Causes and Consequences of Expanded Voluntary Disclosure. Working paper Harvard Business School.

[60] Henry, E. (2008). The Informational Role of Supermajorities. Journal of Public Economics, 92: 2225-2239.

[61] Herrera, H., Llorente-Saguer, A., and McMurray, J. (2019). The Marginal Voter's Curse, Economic Journal, 129 (624): 3137â€“3153.

[62] Hirschman, A. (1981). Exit, Voice, and Loyalty: Responses to Decline in Firms, Organizations, and States, Cambridge, MA: Harvard University Press.

[63] Holderness, C.G. (2009). The Myth of Diffuse Ownership in the United States. Review of Financial Studies, 22 (4), 1377-1408.

[64] Iliev, P., J. Kalodimos, and M. Lowry (2019). Investors' Attention to Corporate Governance, mimeo.

[65] Iliev, P. and M. Lowry (2015). Are Mutual Funds Active Voters? Review of Financial Studies, 28(2): 446-485. 
[66] Kandel, E., M. Massa, and A. Simonov (2011). Do Small Shareholders Count? Journal of Financial Economics, 101: 641-665.

[67] Kim, O., and Verrecchia, R. (1991b). Trading volume and price reactions to public announcements. Journal of Accounting Research, 29, 302-321.

[68] Knyazeva, A., D. Knyazeva, and L. Kostovetsky (2018). Investor Heterogeneity and Trading. European Financial Managerment, 24: 680-718.

[69] Levit, D. and N. Malenko (2011). Nonbinding Voting for Shareholder Proposals. Journal of Finance, LXVI(5): 1579-1614.

[70] Levit, D., N. Malenko, and E. Maug (2021a). The Voting Premium. ECGI Finance Working Paper, 720/2021.

[71] Levit, D., N. Malenko, and E. Maug (2021b). Trading and Shareholder Democracy. ECGI Finance Working Paper, 631/2019.

[72] Li, K. T. Liu, J. Wu (2018). Vote Avoidance and Shareholder Voting in Mergers and Acquisitions, Review of Financial Studies, 31(8): 3176-3211.

[73] Li, S., E. Maug, and M. Schwartz-Ziv (2021). When Shareholders Disagree: Trading after Shareholder Meetings. ECGI Finance Working Paper, 594/2019.

[74] Ma, S., and Y. Xiong (2020). Information Bias in the Proxy Advisory Market, Review of Corporate Finance Studies, 10(1): 82-135.

[75] Malenko, A. and N. Malenko (2019). Proxy Advisory Firms: The Economics of Selling Information to Voters. Journal of Finance, 74(5): 2441-2490.

[76] Mandler, M. (2012), The Fragility of Information Aggregation in Large Elections, Games and Economic Behavior, 74(1): 257-268

[77] Marquez, R. and B. Yilmaz (2008). Information and Efficiency in Tender Offers. Econometrica, 76(5): 1075-1101.

[78] Martinelli, C. (2006). Would Rational Voters Acquire Costly Information?, Journal of Economic Theory, 129: 225-251.

[79] Maskin E, Dasgupta P (1986). The Existence of Equilibrium in Discontinuous Economic Games, Part I (Theory). Review of Economic Studies, 53(1) :1-26. 
[80] Maug, E. and K. Rydqvist (2009). Do Shareholders Vote Strategically? Voting Behavior, Proposal Screening, and Majority Rules, Review of Finance, 13 47-79.

[81] Maug, E. and B. Yilmaz (2002). Two-Class Voting: A Mechanism for Conflict Resolution. American Economic Review, 92(5): 1448-1471.

[82] McLennan, A. (1998). Consequences of the Condorcet Jury Theorem for Beneficial Information Aggregation by Rational Agents, American Political Science Review, 92: 413-418.

[83] McMurray, J. (2013). Aggregating Information by Voting: The Wisdom of the Experts versus the Wisdom of the Masses, Review of Economic Studies, 80(1): 277-312.

[84] Meirowitz, A. and S. Pi (2020). Voting and Trading: The Shareholder's Dilemma. mimeo.

[85] Monderer, D., and Shapley, L. S. (1996). Potential games. Games and Economic Behavior, 14(1), 124-143.

[86] Myers, S. and N. Majluf (1984). Corporate Financing and Investment Decisions When Firms Have Information the Investors Do Not Have. NBER Working Paper, 1396.

[87] Myerson, R. (1998). Population Uncertainty and Poisson Games, International Journal of Game Theory, 27: 375-392.

[88] Nitzan, S. and J. Paroush (1982). Optimal decision rules in uncertain dichotomous choice situations. International Economic Review, 289-297.

[89] Nunez, M. and J.-F. Laslier (2014). Preference Intensity Representation: Strategic Overstating in Large Elections. Social Choice and Welfare, 42: 313-340.

[90] Persico, N. (2004). Committee Design with Endogenous Information, Review of Economic Studies, 71: 165-191.

[91] Richardson, V. (2000). Information Asymmetry and Earnings Management: Some Evidence, Review of Quantitative Finance and Accounting, 15: 325-347.

[92] Sias, R., Starks, L., \& Titman, S. (2006). Changes in institutional ownership and stock returns: Assessment and methodology. Journal of Business, 79, 28692910. 
[93] Schultz, P. (2000). Stock Splits, Tick Size and Sponsorship. Journal of Finance, 55: 429-450.

[94] Schwartz-Ziv, M. and E. Volkova (2020). Is Blockholder Diversity Detrimental. mimeo.

[95] Tirole, J. (2005). The Theory of Corporate Finance, Princeton, New Jersey :Princeton University Press.

[96] Welker, M. (1995). Disclosure Policy, Information Asymmetry and Liquidity in Equity Markets. Contemporary Accounting Research, 11: 801-827.

[97] Woidtke, T. (2002). Agents Watching Agents? Evidence from Pension Fund Ownership and Firm Value, Journal of Financial Economics, 63: 99-131.

[98] Yermack, D. (2010). Shareholder Voting and Corporate Governance. Annual Review of Financial Economics, 2:103-125. 


\section{Online Appendix}

\section{Appendix A: Proofs for Section 4}

Proof of Lemma 1. First, note that, by Bayes' rule:

$$
\mathbb{P}(\alpha \mid \mathbf{s})=\frac{\prod_{i \in N} f_{i}\left(s_{i} \mid \alpha\right) \mathbb{P}(\alpha)}{\prod_{i \in N} f_{i}\left(s_{i} \mid \alpha\right) \mathbb{P}(\alpha)+\prod_{i \in N} f_{i}\left(s_{i} \mid \beta\right) \mathbb{P}(\beta)} .
$$

Thus, $\mathbb{P}(\alpha \mid \mathbf{s})>1 / 2$ requires $\prod_{i \in N} f_{i}\left(s_{i} \mid \alpha\right)>\prod_{i \in N} f_{i}\left(s_{i} \mid \beta\right)$, or equivalently $\sum_{i \in N} \ln \left(\frac{f_{i}\left(s_{i} \mid \alpha\right)}{f_{i}\left(s_{i} \mid \beta\right)}\right)>$ 0 .

Proof of Proposition 1 . In the sequel, we denote the conditional density of shareholder $i$ 's type $t_{i}$ in state $\omega$ by $g_{i}\left(t_{i} \mid \omega\right)$, it is defined by $g_{i}\left(t_{i} \mid \omega\right)=f_{i}\left(\left(s_{i}\right)^{-1}\left(t_{i}\right) \mid \omega\right)$. We denote by $G_{i}(\cdot \mid \omega)$ the corresponding cumulative distribution function. Moreover, we denote the (unconditional) density associated to shareholder $i$ 's type $t_{i}$ by $g_{i}\left(t_{i}\right)$, i.e. defined by $g_{i}\left(t_{i}\right)=\mathbb{P}(\alpha) g_{i}\left(t_{i} \mid \alpha\right)+\mathbb{P}(\beta) g_{i}\left(t_{i} \mid \beta\right)$.

We know from Lemma 4 (in this Appendix) that the best BNE under $X$ is weakly better than the best BNE under $X^{\prime}$. Applying Lemma 5 (in this Appendix), we obtain that the worst BNE under $X, \sigma$, yields at least an expected utility of 0 to each shareholder. As there exists a BNE under $X^{\prime}, \sigma^{\prime}$, which exactly yields an expected utility of 0 to each shareholder (as there is no decisive shareholder by assumption, any profile yielding a sure outcome with no decisive shareholder is an equilibrium), we also obtain that the worst BNE under $X$ (that is, $\sigma$ ) is no worse than the worst BNE under $X^{\prime}$ (that is, $\sigma^{\prime}$ ).

Lemma 4 Let $X$ and $X^{\prime}$ be finite voting rules with $X_{i}^{\prime} \subseteq X_{i}$ for all $i$. Then, for any $B N E \sigma^{\prime}$ under $X^{\prime}$, there exists a BNE $\sigma$ under $X$ such that the ex-ante probability of implementing the correct outcome is (weakly) higher at $\sigma$ than at $\sigma^{\prime}$.

Proof. As the utility of every shareholder is linearly increasing with the probability that the correct outcome is implemented, we employ in this proof as in the later proofs the terms "welfare" and "more efficient" to refer to this common utility. The main task of the proof is to show that a welfare-maximizing (and thus a BNE) exists for any finite voting rule $X$. This is shown in two steps.

Claim 1: for any profile $\sigma$, there is a profile $\sigma^{\prime}$ such that $u_{i}\left(\sigma^{\prime}\right) \geq u_{i}(\sigma)$ and where for all $i \in N, \sigma_{i}^{\prime}: T_{i} \rightarrow X_{i}$ is a pure, weakly increasing strategy. 
For any $i \in N$, we may write:

$$
\begin{aligned}
\mathbb{E}\left[u_{i}\left(x, \sigma_{-i}\right) \mid t_{i}\right]=h_{i} & \times\left(\mathbb{P}\left(\alpha \mid t_{i}\right)\left(\mathbb{P}_{\sigma_{-i}}\left(\sum_{j \neq i} x_{j}>-x \mid \alpha\right)+\frac{1}{2} \mathbb{P}_{\sigma_{-i}}\left(\sum_{j \neq i} x_{j}=-x \mid \alpha\right)\right)\right. \\
& \left.-\left(1-\mathbb{P}\left(\alpha \mid t_{i}\right)\right)\left(\mathbb{P}_{\sigma_{-i}}\left(\sum_{j \neq i} x_{j}>-x \mid \beta\right)+\frac{1}{2} \mathbb{P}_{\sigma_{-i}}\left(\sum_{j \neq i} x_{j}=-x \mid \beta\right)\right)\right) .
\end{aligned}
$$

Hence,

$$
\begin{array}{r}
\frac{\partial \mathbb{E}\left[u_{i}\left(x, \sigma_{-i}\right) \mid t_{i}\right]}{\partial t_{i}}=h_{i} \times \frac{\partial \mathbb{P}\left(\alpha \mid t_{i}\right)}{\partial t_{i}}\left(\mathbb{P}_{\sigma_{-i}}\left(\sum_{j \neq i} x_{j}>-x \mid \alpha\right)+\mathbb{P}_{\sigma_{-i}}\left(\sum_{j \neq i} x_{j}>-x \mid \beta\right)\right. \\
\left.+\frac{1}{2} \mathbb{P}_{\sigma_{-i}}\left(\sum_{j \neq i} x_{j}=-x \mid \alpha\right)+\frac{1}{2} \mathbb{P}_{\sigma_{-i}}\left(\sum_{j \neq i} x_{j}=-x \mid \beta\right)\right) .
\end{array}
$$

We have $\mathbb{P}\left(\alpha \mid t_{i}\right)=\frac{g_{i}\left(t_{i} \mid \alpha\right)}{g_{i}\left(t_{i} \mid \alpha\right)+g_{i}\left(t_{i} \mid \beta\right)}=\frac{t_{i}}{1+t_{i}}=1-\frac{1}{1+t_{i}}$, and we get $\frac{\partial \mathbb{P}\left(\alpha \mid t_{i}\right)}{\partial t_{i}}=\frac{1}{\left(1+t_{i}\right)^{2}}>0$. This implies that $\frac{\partial \mathbb{E}\left[u_{i}\left(x, \sigma_{-i}\right) \mid t_{i}\right]}{\partial t_{i}}$ is weakly increasing in $x$ (increasing differences). As $X_{i}$ is finite, let us define the best reply $\sigma_{i}^{\prime}$ to $\sigma_{-i}$ as the smallest value of $x \in X_{i}$ maximizing the expected utility (we abuse notation as $\sigma_{i}^{\prime}$ is a pure strategy):

$$
\forall t_{i} \in T_{i}, \quad \sigma_{i}^{\prime}\left(t_{i}\right)=\min \left\{x \in X_{i} \mid \mathbb{E}\left[u_{i}\left(x, \sigma_{-i}\right) \mid t_{i}\right] \geq \mathbb{E}\left[u_{i}\left(y, \sigma_{-i}\right) \mid t_{i}\right], \quad \forall y \in X_{i}\right\}
$$

The strategy $\sigma_{i}^{\prime}$ must be weakly increasing. Assume by contradiction that $x=\sigma_{i}^{\prime}\left(t_{i}\right)>y=$ $\sigma_{i}^{\prime}\left(t_{i}^{\prime}\right)$ for $t_{i}<t_{i}^{\prime}$. Then, by definition of $\sigma_{i}^{\prime}\left(t_{i}\right)$ as a minimum, we have $\mathbb{E}\left[u_{i}\left(x, \sigma_{-i}\right) \mid t_{i}\right]>$ $\mathbb{E}\left[u_{i}\left(y, \sigma_{-i}\right) \mid t_{i}\right]$ and thus, using the property of increasing differences, we obtain :

$$
\begin{aligned}
\mathbb{E}\left[u_{i}\left(x, \sigma_{-i}\right) \mid t_{i}^{\prime}\right) & =\mathbb{E}\left[u_{i}\left(x, \sigma_{-i}\right) \mid t_{i}\right]+\int_{t_{i}}^{t_{i}^{\prime}} d t \frac{\partial \mathbb{E}\left[u_{i}\left(x, \sigma_{-i}\right) \mid t\right]}{\partial t} \\
& >\mathbb{E}\left[u_{i}\left(y, \sigma_{-i}\right) \mid t_{i}\right]+\int_{t_{i}}^{t_{i}^{\prime}} d t \frac{\partial \mathbb{E}\left[u_{i}\left(y, \sigma_{-i}\right) \mid t\right]}{\partial t}=\mathbb{E}\left[u_{i}\left(y, \sigma_{-i}\right) \mid t_{i}^{\prime}\right],
\end{aligned}
$$

a contradiction with $y$ being a best reply at $t_{i}^{\prime}$. Hence, the (pure) strategy $\sigma_{i}^{\prime}$ is weakly increasing in $t_{i}^{\prime}$. By applying the same reasoning iteratively for $i=1, \ldots, n$, we obtain the profile $\sigma^{\prime}$, as desired.

Claim 2: the rule $X$ admits a welfare-maximizing strategy profile, which is thus a BNE.

Let us consider the family of profiles consisting in pure, weakly increasing strategies. Let us write $X_{i}=\left\{x^{1}, \ldots, x^{k}\right\}$ with $x^{1}<\ldots<x^{k}$. A pure, weakly increasing strategy $\sigma_{i}$ is thus described by a series of cutoffs $\left(t_{i}^{j}\right)_{0 \leq j \leq k} \in\left(T_{i}\right)^{k+1}$, with $t_{i}^{0}=\delta_{i}$ and $t_{i}^{k}=\frac{1}{\delta_{i}}$, and such that $\forall j, t_{i}^{j} \leq t_{i}^{j+1}$ and $t_{i} \in\left(t_{i}^{j}, t_{i}^{j+1}\right) \Rightarrow \sigma_{i}\left(t_{i}\right)=x^{j+1}$. A profile of such strategies is 
thus described by a series of cutoffs for each shareholder $i \in N$. Now, as each distribution $G_{i}(\cdot \mid \omega)$ does not admit any atom, the expected utility attached to such profile is a continuous function of its cutoffs. As cutoffs are taken in a compact set, there is a profile $\sigma^{*}$ maximizing the expected utility among all profiles in the family. By application of Claim $1, \sigma^{*}$ maximizes the expected utility among all profiles. As the game is of common interest, the profile $\sigma^{*}$ must be a $\mathrm{BNE}$, and hence a welfare-maximizing BNE (this is the original argument of Mc Lennan, 1998).

Finally, to conclude, whenever two rules $X$ and $X^{\prime}$ are such that $\forall i \in N, X_{i}^{\prime} \subseteq X_{i}$, then each profile under rule $X^{\prime}$ can be reproduced under rule $X$. It follows that the welfare-maximizing profile (BNE) $\sigma$ under $X$ achieves at least as much expected utility as the welfare-maximizing profile (BNE) $\sigma^{\prime}$ under $X^{\prime}$. This concludes the proof. 
Lemma 5 For any BNE $\sigma$ under a finite rule $X$, we have $\forall i \in N, u_{i}(\sigma) \geq 0$.

Proof. We introduce a couple of notations for the proof. For a strategy profile $\sigma$ and a type vector $\mathbf{t}=\left(t_{i}\right)_{i \in N}$, we denote by $p^{A}(\sigma(\mathbf{t}))$ the probability that $A$ is implemented given the votes $\sigma(t)$ :

$$
p^{A}(\sigma(\mathbf{t}))=\mathbb{P}\left(\sum_{i \in N} \widehat{\sigma}_{i}\left(t_{i}\right)>0\right)+\frac{1}{2} \mathbb{P}\left(\sum_{i \in N} \widehat{\sigma}_{i}\left(t_{i}\right)=0\right) .
$$

Note that we have $\int p^{A}(\sigma(\mathbf{t})) \prod_{i=1}^{n} g_{i}\left(t_{i}\right) d t_{i}=\mathbb{P}(O=A \mid \sigma)$.

Claim 1: For any profile of pure, weakly increasing strategies $\sigma$, we have $u_{i}(\sigma) \geq 0$ for all $i$.

Let $\sigma$ be a profile of pure, weakly increasing strategies. Let us denote by $U(\sigma)=$ $u_{i}(\sigma) / h_{i}$ the common utility. We may then write:

$$
\begin{aligned}
U(\sigma) & =\int(\mathbb{P}(\alpha \mid \mathbf{t})-\mathbb{P}(\beta \mid \mathbf{t})) p^{A}(\sigma(\mathbf{t})) \prod_{i=1}^{n} g_{i}\left(t_{i}\right) d t_{i} \\
& =\int(2 \mathbb{P}(\alpha \mid \mathbf{t})-1) p^{A}(\sigma(\mathbf{t})) \prod_{i=1}^{n} g_{i}\left(t_{i}\right) d t_{i} \\
& =2 \underbrace{\int \mathbb{P}(\alpha \mid \mathbf{t}) p^{A}(\sigma(\mathbf{t})) \prod_{i=1}^{n} g_{i}\left(t_{i}\right) d t_{i}}_{\widetilde{U}(\sigma)}-\mathbb{P}(O=A \mid \sigma) .
\end{aligned}
$$

To prove the claim that $U(\sigma) \geq 0$, it thus suffices to show that $\widetilde{U}(\sigma) \geq \frac{1}{2} \mathbb{P}(O=A \mid \sigma)$. We first observe that, for any $k \in N$, the function $g^{k}: t_{k} \mapsto \int \mathbb{P}(\alpha \mid \mathbf{t}) \prod_{i=1}^{k-1} g_{i}\left(t_{i}\right) d t_{i}$ is weakly increasing (for each $t_{-k}$ ). Moreover, for any $k \in N$, the function $h^{k}: t_{k} \mapsto$ $\int p^{A}(\sigma(\mathbf{t})) \prod_{i=1}^{k-1} g_{i}\left(t_{i}\right) d t_{i}$ is weakly increasing (for each $t_{-k}$ ) since $\sigma_{k}$ is weakly increasing. 
By repeated application of Lemma 6, we thus obtain:

$$
\begin{aligned}
\widetilde{U}(\sigma) & =\int \mathbb{P}(\alpha \mid \mathbf{t}) p^{A}(\sigma(\mathbf{t})) \prod_{i=1}^{n} g_{i}\left(t_{i}\right) d t_{i} \\
& \geq \int\left(\int \mathbb{P}(\alpha \mid \mathbf{t}) g_{1}\left(t_{1}\right) d t_{1}\right) \times\left(\int p^{A}(\sigma(\mathbf{t})) g_{1}\left(t_{1}\right) d t_{1}\right) \prod_{i=2}^{n} g_{i}\left(t_{i}\right) d t_{i} \\
& \geq \cdots \\
& \geq \int\left(\int \mathbb{P}(\alpha \mid \mathbf{t}) \prod_{i=1}^{k} g_{i}\left(t_{i}\right) d t_{i}\right) \times\left(\int p^{A}(\sigma(\mathbf{t})) \prod_{i=1}^{k} g_{i}\left(t_{i}\right) d t_{i}\right) \prod_{i=k+1}^{n} g_{i}\left(t_{i}\right) d t_{i} \\
& \geq \ldots \\
& \geq\left(\int \mathbb{P}(\alpha \mid \mathbf{t}) \prod_{i=1}^{n} g_{i}\left(t_{i}\right) d t_{i}\right) \times\left(\int p^{A}(\sigma(\mathbf{t})) \prod_{i=1}^{n} g_{i}\left(t_{i}\right) d t_{i}\right)=\frac{1}{2} \mathbb{P}(O=A \mid \sigma) .
\end{aligned}
$$

This concludes the proof of Claim 1.

Claim 2: For any BNE $\sigma$, there exists a profile $\sigma^{+}$in pure, weakly increasing strategies such that $\forall i \in N, u_{i}(\sigma)=u_{i}\left(\sigma^{+}\right)$.

Let $\sigma$ be a BNE. For any strategy $\sigma_{i}$ of shareholder $i$, we consider a re-ordering $\sigma_{i}^{+}$, i.e. a strategy such that:

- $\sigma_{i}^{+}$is pure and weakly increasing

- for any ballot $x_{i} \in X_{i}$, we have $\mathbb{P}\left(\widehat{\sigma}_{i} \leq x_{i}\right)=\mathbb{P}\left(\sigma_{i}^{+} \leq x_{i}\right)$.

To construct such a re-ordering, we define $\sigma_{i}^{+}$by (abusing notation as $\sigma_{i}^{+}$is pure):

$$
\forall t_{i} \in T_{i}, \quad \sigma_{i}^{+}\left(t_{i}\right)=\min \left\{x_{i} \in X_{i} \mid \sum_{x \in X_{i}, x \leq x_{i}} \int_{\delta_{i}}^{\frac{1}{\delta_{i}}} \sigma_{i}\left(t_{i}^{\prime}\right)(x) g_{i}\left(t_{i}^{\prime}\right) d t_{i}^{\prime}>\int_{\delta_{i}}^{t_{i}} g_{i}\left(t_{i}^{\prime}\right) d t_{i}^{\prime}\right\} .
$$

The strategy $\sigma_{i}^{+}$is pure, weakly increasing and continuous (and even flat) everywhere but on a finite number of points. We shall prove that for almost any type vector $\mathbf{t}=\left(t_{i}\right)_{i \in N}$, the sign of $\sum_{i \in N} \widehat{\sigma}_{i}\left(t_{i}\right)$ is the same as that of $\sum_{i \in N} \sigma_{i}^{+}\left(t_{i}\right)$. In the sequel, we refer to the sign of a number $x$ as positive if $x>0$, negative if $x<0$, and null (neither positive nor negative) if $x=0$.

Let $t_{i} \in T_{i}$ be a type such that $\sigma_{i}^{+}$is continuous at $t_{i}$ and assume that there exists $x_{i} \in X_{i}$ for which $\sigma_{i}\left(t_{i}\right)\left(x_{i}\right)>0$ and $x_{i} \neq \sigma_{i}^{+}\left(t_{i}\right)$. We focus on the case for which $\sigma_{i}^{+}\left(t_{i}\right)>x_{i}$ (the other case can be treated analogously) and we further assume that $x_{i}=\min \left\{x \in X_{i} \mid \sigma_{i}\left(t_{i}\right)(x)>0\right\}:=\min \widehat{\sigma}_{i}\left(t_{i}\right)$. Observe that there must exist $t_{i}^{\prime}<t_{i}$ such that $\sigma_{i}\left(t_{i}^{\prime}\right)\left(y_{i}\right)>0$ with $y_{i} \geq \sigma_{i}^{+}\left(t_{i}\right)>x_{i}$. Indeed, if this type $t_{i}^{\prime}$ didn't exist, we would 
have $\forall t_{i}^{\prime}<t_{i}, \widehat{\sigma}_{i}\left(t_{i}^{\prime}\right)<\sigma_{i}^{+}\left(t_{i}\right)$ for any realization of $\sigma_{i}\left(t_{i}^{\prime}\right)$, which would imply:

$$
\sum_{x<\sigma_{i}^{+}\left(t_{i}\right)} \int_{\delta_{i}}^{\frac{1}{\delta_{i}}} \sigma_{i}\left(t{ }_{i}\right)(x) g_{i}\left(t{ }_{i}\right) d t{ }_{i} \geq \int_{\delta_{i}}^{t_{i}}\left(\sum_{x<\sigma_{i}^{+}\left(t_{i}\right)} \sigma_{i}\left(t{ }_{i}\right)(x)\right) g_{i}\left(t{ }_{i}\right) d t{ }_{i}=\int_{\delta_{i}}^{t_{i}} g_{i}\left(t{ }_{i}\right) d t{ }_{i} .
$$

We would then have for any $t<t_{i}$ ( since $g_{i}$ is positive on $T_{i}$ ):

$$
\sum_{x<\sigma_{i}^{+}\left(t_{i}\right)} \int_{\delta_{i}}^{\frac{1}{\delta_{i}}} \sigma_{i}\left(t{ }^{\prime}{ }_{i}\right)(x) g_{i}\left(t{ }_{i}\right) d t{ }_{i}>\int_{\delta_{i}}^{t} g_{i}\left(t{ }_{i}\right) d t{ }_{i}
$$

By definition of $\sigma_{i}^{+}$, we would have $\forall t<t_{i}, \sigma_{i}^{+}(t)<\sigma_{i}^{+}\left(t_{i}\right)$. This contradicts the fact that $\sigma_{i}^{+}$is continuous at $t_{i}$. We thus obtained the existence of $y_{i} \geq \sigma_{i}^{+}\left(t_{i}\right)>x_{i}$ such that $\sigma_{i}\left(t_{i}^{\prime}\right)\left(y_{i}\right)>0$ for some $t_{i}^{\prime}<t_{i}$.

As $\sigma$ is a BNE, $x_{i}$ must be optimal for $i$ at $t_{i}$ and $y_{i}$ must be optimal for $i$ at $t_{i}^{\prime}$ :

$$
\begin{aligned}
& \Delta_{i}:=\frac{1}{h_{i}}\left(u_{i}\left(x_{i}, \sigma_{-i} \mid t_{i}\right)-u_{i}\left(y_{i}, \sigma_{-i} \mid t_{i}\right)\right) \geq 0 \\
& \Delta_{i}^{\prime}:=\frac{1}{h_{i}}\left(u_{i}\left(y_{i}, \sigma_{-i} \mid t_{i}^{\prime}\right)-u_{i}\left(x_{i}, \sigma_{-i} \mid t_{i}^{\prime}\right)\right) \geq 0 .
\end{aligned}
$$

By summation, we obtain that $\Delta_{i}+\Delta_{i}^{\prime} \geq 0$. Now, we may write:

$$
\Delta_{i}=\int\left(2 \mathbb{P}\left(\alpha \mid t_{i}, t_{-i}\right)-1\right)\left(p^{A}\left(x_{i}, \sigma_{-i}\left(t_{-i}\right)\right)-p^{A}\left(y_{i}, \sigma_{-i}\left(t_{-i}\right)\right)\right) \prod_{j \neq i} g_{j}\left(t_{j}\right) d t_{j} .
$$

Similarly,

$$
\Delta_{i}^{\prime}=\int\left(2 \mathbb{P}\left(\alpha \mid t_{i}^{\prime}, t_{-i}\right)-1\right)\left(p^{A}\left(y_{i}, \sigma_{-i}\left(t_{-i}\right)\right)-p^{A}\left(x_{i}, \sigma_{-i}\left(t_{-i}\right)\right)\right) \prod_{j \neq i} g_{j}\left(t_{j}\right) d t_{j} .
$$

We thus have:

$$
\begin{aligned}
\Delta_{i}+\Delta_{i}^{\prime} & =2 \int\left(\mathbb{P}\left(\alpha \mid t_{i}^{\prime}, t_{-i}\right)-\mathbb{P}\left(\alpha \mid t_{i}, t_{-i}\right)\right)\left(p^{A}\left(y_{i}, \sigma_{-i}\left(t_{-i}\right)\right)-p^{A}\left(x_{i}, \sigma_{-i}\left(t_{-i}\right)\right)\right) \prod_{j \neq i} g_{j}\left(t_{j}\right) d t_{j} \\
& \geq 0
\end{aligned}
$$

As $t_{i}^{\prime}<t_{i}$, we have that for all $t_{-i}, \mathbb{P}\left(\alpha \mid t_{i}^{\prime}, t_{-i}\right)-\mathbb{P}\left(\alpha \mid t_{i}, t_{-i}\right)<0$. Moreover, as $y_{i}>x_{i}$, we have by definition of $p^{A}$ that for all $t_{-i}, p^{A}\left(y_{i}, \sigma_{-i}\left(t_{-i}\right)\right) \geq p^{A}\left(x_{i}, \sigma_{-i}\left(t_{-i}\right)\right)$. To reconcile the three inequalities, it must be that $\Delta_{i}+\Delta_{i}^{\prime}=0$ and that for almost all $t_{-i}$, $p^{A}\left(y_{i}, \sigma_{-i}\left(t_{-i}\right)\right)=p^{A}\left(x_{i}, \sigma_{-i}\left(t_{-i}\right)\right)$. This last equality implies, by definition of $p^{A}$, that 
$\mathbb{P}\left(x_{i} \leq-\sum_{j \neq i} \widehat{\sigma}_{j} \leq y_{i}\right)=0$. As $x_{i}=\min \widehat{\sigma}_{i}\left(t_{i}\right)$ and $\sigma_{i}^{+}\left(t_{i}\right) \leq y_{i}$, we obtain

$$
\mathbb{P}\left(\min \widehat{\sigma}_{i}\left(t_{i}\right) \leq-\sum_{j \neq i} \widehat{\sigma}_{j} \leq \sigma_{i}^{+}\left(t_{i}\right)\right)=0 .
$$

Following a symmetrical argument, we also obtain

$$
\mathbb{P}\left(\sigma_{i}^{+}\left(t_{i}\right) \leq-\sum_{j \neq i} \widehat{\sigma}_{j} \leq \max \widehat{\sigma}_{i}\left(t_{i}\right)\right)=0 .
$$

It follows that $\mathbb{P}\left(\operatorname{sgn}\left(\widehat{\sigma}_{i}\left(t_{i}\right)+\sum_{j \neq i} \widehat{\sigma}_{j}\right) \neq \operatorname{sgn}\left(\sigma_{i}^{+}\left(t_{i}\right)+\sum_{j \neq i} \widehat{\sigma}_{j}\right)\right)=0$. Moreover, by construction of the strategies $\left(\sigma_{j}^{+}\right)_{j \neq i}$, the probability of the previous event remains null if some strategy realizations $\widehat{\sigma}_{j}$ are transformed into $\sigma_{j}^{+}$(the transformation from $\sigma_{j}$ to $\sigma_{j}^{+}$is measure-preserving by design). This can be written: for all $S \subseteq N \backslash\{i\}$,

$\mathbb{P}\left(\operatorname{sgn}\left(\widehat{\sigma}_{i}\left(t_{i}\right)+\sum_{j \in S} \widehat{\sigma}_{j}+\sum_{j \in(N \backslash S) \backslash\{i\}} \sigma_{j}^{+}\right) \neq \operatorname{sgn}\left(\sigma_{i}^{+}\left(t_{i}\right)+\sum_{j \in S} \widehat{\sigma}_{j}+\sum_{j \in(N \backslash S) \backslash\{i\}} \sigma_{j}^{+}\right)\right)=0$.

We have just shown that (1) holds whenever $\sigma_{i}^{+}$is continuous at $t_{i}$. As $\sigma_{i}^{+}$is continuous almost everywhere, we have: for all $S \subseteq N \backslash\{i\}$,

$$
\mathbb{P}\left(\operatorname{sgn}\left(\widehat{\sigma}_{i}+\sum_{j \in S} \widehat{\sigma}_{j}+\sum_{j \in(N \backslash S) \backslash\{i\}} \sigma_{j}^{+}\right) \neq \operatorname{sgn}\left(\sigma_{i}^{+}+\sum_{j \in S} \widehat{\sigma}_{j}+\sum_{j \in(N \backslash S) \backslash\{i\}} \sigma_{j}^{+}\right)\right)=0 .
$$

To conclude, we observe that $\operatorname{sgn}\left(\sum_{j \in N} \widehat{\sigma}_{j}\right) \neq \operatorname{sgn}\left(\sum_{j \in N} \sigma_{j}^{+}\right)$can be satisfied only if there exists some index $k$ for which $\operatorname{sgn}\left(\sum_{j=1}^{k-1} \widehat{\sigma}_{j}+\sum_{j=k}^{n} \sigma_{j}^{+}\right) \neq \operatorname{sgn}\left(\sum_{j=1}^{k} \widehat{\sigma}_{j}+\sum_{j=k+1}^{n} \sigma_{j}^{+}\right)$. Hence, we may write, applying (2):

$\mathbb{P}\left(\operatorname{sgn}\left(\sum_{j \in N} \widehat{\sigma}_{j}\right) \neq \operatorname{sgn}\left(\sum_{j \in N} \sigma_{j}^{+}\right)\right) \leq \sum_{k=1}^{n} \mathbb{P}\left(\operatorname{sgn}\left(\sum_{j=1}^{k-1} \widehat{\sigma}_{j}+\sum_{j=k}^{n} \sigma_{j}^{+}\right) \neq \operatorname{sgn}\left(\sum_{j=1}^{k} \widehat{\sigma}_{j}+\sum_{j=k+1}^{n} \sigma_{j}^{+}\right)\right)=0$.

Therefore, $\sigma$ and $\sigma^{+}$lead to the same outcome with probability one, i.e. for almost any type vector $t$. It follows that $\forall i \in N, u_{i}(\sigma)=u_{i}\left(\sigma^{+}\right)$. This concludes the proof of Claim 2.

To conclude the proof, note that any BNE $\sigma$ yields the same utilities as a profile $\sigma^{+}$of pure, weakly increasing strategies (Claim 2), under which all expected utilities are positive (Claim 1). Thus for all $i \in N, u_{i}(\sigma) \geq 0$. 
Lemma 6 Let $f$ be a density function on a real interval $T$, let $g, h: T \rightarrow \mathbb{R}$ be two weakly increasing functions. Then:

$$
\int g(t) h(t) f(t) d t \geq\left(\int g(t) f(t) d t\right) \times\left(\int h(t) f(t) d t\right) .
$$

Proof. The statement is a direct consequence of the property that, for any real random variable $Y$ (drawn with density $f$ ) and for any pair of weakly increasing functions $(g, h)$, we have:

$$
\operatorname{Cov}[g(Y), h(Y)]=\mathbb{E}[g(Y) h(Y)]-\mathbb{E}[g(Y)] \mathbb{E}[h(Y)] \geq 0
$$

Proof of Proposition 2. The fact that each $i \in N$ employing $\sigma_{i}^{1 S 1 V-D}\left(t_{i}\right)=c \ln t_{i}$ with $c=\min _{i \in N}\left\{\frac{-d_{i}}{\ln (\delta)}\right\}$ is an efficient BNE of the game is straightforward, by application of Lemma 1. We can easily rule out the existence of a non-degenerate mixed efficient BNE. If $\sigma$ is a non-degenerate mixed BNE of the game, there exists at least one $i \in N$ and at least one $y \in T$ such that the random variable $\sigma_{i}(y)$ admits at least two distinct potential realizations. Consider without loss of generality that this player is the first shareholder. If $\sigma$ is efficient then for $\mathbf{t}=\left(t_{1}, t_{2}, t_{3}, t_{4}, \ldots\right)=\left(y, \frac{1}{y}, 1,1, \ldots\right)$ we have that the efficient alternative is $A B$. Hence, given any two vectors of potential realizations $\widehat{\sigma}=$ $\left(\widehat{\sigma}_{1}(y), \widehat{\sigma}_{2}\left(\frac{1}{y}\right), \widehat{\sigma}_{3}(1), \widehat{\sigma}_{4}(1), \ldots\right)$ and $\widetilde{\sigma}=\left(\widetilde{\sigma}_{1}(y), \widetilde{\sigma}_{2}\left(\frac{1}{y}\right), \widetilde{\sigma}_{3}(1), \widetilde{\sigma}_{4}(1), \ldots\right)$ with $\widehat{\sigma}_{i}\left(t_{i}\right)=\widetilde{\sigma}_{i}\left(t_{i}\right)$ for every $i>1$, we must have that $\sum_{i \in N} \widehat{\sigma}\left(t_{i}\right)=0$ and $\sum_{i \in N} \tilde{\sigma}\left(t_{i}\right)=0$. But this means that $\widehat{\sigma}_{1}(y)$ must be identical to $\widetilde{\sigma}_{1}(y)$ and hence $\sigma_{1}(y)$ cannot admit at least two distinct potential realizations, which contradicts the assumption above. Hence, if $\sigma$ is an efficient BNE, it must be pure.

We now turn attention to pure equilibria. First we argue that an efficient pure BNE $\sigma$ must be symmetric across shareholders up to an additive constant (i.e. there exist $\phi_{i, j}$ such that $\sigma_{i}(y)=\sigma_{j}(y)+\phi_{i, j}$, for every $i, j \in N$ and every $\left.y \in T\right)$. If $\sigma$ is efficient then for every $y \in T$, for $\mathbf{t}=\left(t_{1}, t_{2}, t_{3}, t_{4}, \ldots\right)$ with $t_{i}=y, t_{j}=\frac{1}{y}$ and $t_{k}=1$ for all $k \notin\{i, j\}$, we need to have $\sum_{k \in N} \sigma_{k}\left(t_{k}\right)=0$ and thus $\sigma_{i}(y)=-\sigma_{j}\left(\frac{1}{y}\right)-\sum_{k \in N-\{i . j\}} \sigma_{k}(1)$. By keeping $j$ fixed and varying $i$ we get that all players, except possibly $j$, employ the same strategy up to an additive constant. By varying $j$ as well, we get that all players use the same strategy up to an additive constant. That is, in an efficient pure BNE $\sigma$ a player $i$ uses the strategy $\sigma_{i}(y)=\theta(y)+\kappa_{i}$, with $\sum_{i \in N} \kappa_{i}=0$ and $\theta(1)=0$.

Notice that if $\sigma$ is an efficient equilibrium of the standard $1 S 1 \mathrm{~V}-D$ rule characterized by some $\theta$ and $\boldsymbol{\kappa}=\left(\kappa_{1}, \kappa_{2}, . ., \kappa_{n}\right)$ such that $\kappa_{i} \neq 0$ for at least one $i \in N$, it follows that $\sigma^{\prime}$, characterized by the same $\theta$ and $\boldsymbol{\kappa}=(0,0, \ldots, 0)$, is an efficient equilibrium of the rule $v^{\prime}=(\mathbb{R}, \mathbb{R}, \ldots, \mathbb{R})$. Hence, to characterize all efficient equilibria of the standard $1 S 1 V$ - $D$ rule, it suffices to characterize all admissible $\theta$ s that lead to full information equivalence 
when $\kappa=(0,0, \ldots, 0)$ under rule $v^{\prime}$. In the remaining part of the proof, we slightly abuse terminology, and instead of saying "a pure-strategy efficient equilibrium of $v$ characterized by $\theta$ and $\kappa=(0,0, \ldots, 0)$ " we simply say "an efficient equilibrium $\theta$."

In an efficient equilibrium $\theta$, it must be the case that for every $\mathbf{t} \in T^{n}$ we have $\operatorname{sgn}\left(\sum_{i \in N} \theta\left(t_{i}\right)\right)=\operatorname{sgn}(\mathbb{P}(\alpha \mid \mathbf{t})-\mathbb{P}(\beta \mid \mathbf{t}))$. But we know from Lemma 1 that $\mathbb{P}(\alpha \mid \mathbf{t})-$ $\mathbb{P}(\beta \mid \mathbf{t})>0 \Leftrightarrow \sum_{i \in N} \ln \left(t_{i}\right)>0, \mathbb{P}(\alpha \mid \mathbf{t})-\mathbb{P}(\beta \mid \mathbf{t})<0 \Leftrightarrow \sum_{i \in N} \ln \left(t_{i}\right)<0$, and $\mathbb{P}(\alpha \mid \mathbf{t})-$ $\mathbb{P}(\beta \mid \mathbf{t})=0 \Leftrightarrow \sum_{i \in N} \ln \left(t_{i}\right)=0$. In other words, for every $\mathbf{t} \in T^{n}$ it must hold that $\operatorname{sgn}\left(\sum_{i \in N} \theta\left(t_{i}\right)\right)=\operatorname{sgn}\left(\sum_{i \in N} \ln \left(t_{i}\right)\right)$.

First, we prove that every efficient equilibrium $\theta$ is monotone (increasing, in particular) and symmetric (i.e., $\theta(y)=-\theta\left(\frac{1}{y}\right)$ for every $y \in T$ ), then that it is differentiable on $\operatorname{int}(T)=\left(\delta, \frac{1}{\delta}\right)$ and continuous on $T=\left[\delta, \frac{1}{\delta}\right]$, and, finally, we provide a full characterization by showing that each efficient equilibrium $\theta$ is equal to the natural logarithm multiplied by some positive constant.

Monotonicity and symmetry of equilibria: For every $t_{i}<\frac{1}{\delta}$ there exists a $t_{-i} \in$ $T^{n-1}$ such that $\mathbb{P}(\alpha \mid \mathbf{t})=\mathbb{P}(\beta \mid \mathbf{t})$, so that $\sum_{i \in N} \ln \left(t_{i}\right)=0$. Hence, for such a $\mathbf{t}=\left(t_{i}, t_{-i}\right)$ and every $\varepsilon \in\left(0, \frac{1}{\delta}-t_{i}\right]$, it is true that, $\mathbb{P}\left(\alpha \mid\left(t_{i}+\varepsilon, t_{-i}\right)\right)>\mathbb{P}\left(\beta \mid\left(t_{i}+\varepsilon, t_{-i}\right)\right)$, so that $\sum_{j \in N-\{i\}} \ln \left(t_{j}\right)+\ln \left(t_{i}+\varepsilon\right)>0$. Since every efficient equilibrium $\theta$ delivers the efficient outcome, it follows that for every $y<\frac{1}{\delta}$ and $\varepsilon \in\left(0, \frac{1}{\delta}-y\right]$ there exists a $t_{-i} \in T^{n-1}$ such that $\sum_{j \in N-\{i\}} \theta\left(t_{j}\right)+\theta(y)=0$ and $\sum_{j \in N-\{i\}} \theta\left(t_{j}\right)+\theta(y+\varepsilon)>0$. In other words, $\theta(y+\varepsilon)>\theta(y)$ for every $y<\frac{1}{\delta}$ and $\varepsilon \in\left(0, \frac{1}{\delta}-y\right] ; \theta$ is strictly increasing in the player's type. To establish symmetry, consider that $\mathbf{t} \in T^{n}$ is such that $t_{i}=1$ for every $i \in N$. In this case the efficient alternative is $A B$. Therefore, $\sum_{i \in N} \theta\left(t_{i}\right)=n \theta(1)=0$, which implies $\theta(1)=0$. Now consider a $\mathbf{t} \in T^{n}$ such that $t_{1}=y \in T, t_{2}=\frac{1}{y} \in T$ and $t_{i}=1$ for every $i>2$. We have $\sum_{i \in N-\{1,2\}} \ln (1)+\ln (y)+\ln \left(\frac{1}{y}\right)=0$ and hence $\sum_{i \in N-\{1,2\}} \theta(1)+\theta(y)+\theta\left(\frac{1}{y}\right)=0$, which implies $(n-2) \times 0+\theta(y)+\theta\left(\frac{1}{y}\right)=0$, for every $y \in T$. In other words, $\theta(y)=-\theta\left(\frac{1}{y}\right)$, for every $y \in T$.

Differentiability: By Lebesgue's theorem for the differentiability of monotone functions defined over open intervals we have that every equilibrium $\theta: T \rightarrow \mathbb{R}$ is differentiable at almost every $y \in \operatorname{int}(T)=\left(\delta, \frac{1}{\delta}\right)$. We will now establish that $\theta$ is actually differentiable at every $y \in\left(\delta, \frac{1}{\delta}\right)$. Notice that in all profiles with $t_{1}=y \in(\delta, 1]$, $t_{2}=y^{\prime} \in\left(1, \frac{1}{\delta}\right), t_{3}=\frac{1}{y y^{\prime}} \in\left(\delta, \frac{1}{\delta}\right)$, and $t_{i}=1$ for every $i>3$, it must hold that $\theta(y)+\theta\left(y^{\prime}\right)+\theta\left(\frac{1}{y y^{\prime}}\right)=0 \Leftrightarrow \theta(y)=-\theta\left(y^{\prime}\right)-\theta\left(\frac{1}{y y^{\prime}}\right)$ by the fact $\theta(1)=0$ and that $\sum_{i \in N} \ln \left(t_{i}\right)=0$. Assume that $\theta$ is not differentiable at a particular $\tilde{y} \in(\delta, 1]$. Then it follows that $-\theta\left(y^{\prime}\right)-\theta\left(\frac{1}{y y^{\prime}}\right)$ is not differentiable with respect to $y$ at $\tilde{y}$, for every $y^{\prime} \in\left(1, \frac{1}{\delta}\right)$. But due to the fact that $\theta$ is differentiable at almost every $y \in \operatorname{int}(T)$, it follows that for every $y \in(\delta, 1]$, there exists $y^{\prime} \in\left(1, \frac{1}{\delta}\right)$ such that $\theta$ is differentiable at $\frac{1}{y y^{\prime}}$. This contradicts the claim that there exists $\tilde{y} \in(\delta, 1]$ at which $\theta$ is not differentiable, and, by symmetry it follows that $\theta$ is differentiable at every $y \in \operatorname{int}(T)$. 
Continuity at the boundary: We know that $\theta$ is differentiable, and thus continuous, on $\operatorname{int}(T)=\left(\delta, \frac{1}{\delta}\right)$. Let us show that it is continuous at $y=\frac{1}{\delta}$. Suppose by contradiction, that there is a discontinuity. As $\theta$ is increasing, it must be of the form: $\theta(y)-\theta(y-\varepsilon)>\tilde{\varepsilon}$ for every $\varepsilon \in(0, \bar{\varepsilon}]$, where $\bar{\varepsilon}$ and $\tilde{\varepsilon}$ are positive constants. Then there exists $\lambda \in(0,1-\delta)$ such that $(n-2) \theta(1-\lambda)+\theta(y)+\theta\left(\frac{1}{y-\varepsilon}\right)>0$ for every $\varepsilon \in(0, \bar{\varepsilon}]$. But for every $\lambda \in(0,1-\delta)$ one can find $\varepsilon>0$ small enough such that $(n-2) \ln (1-\lambda)+\ln (y)+\ln \left(\frac{1}{y-\varepsilon}\right)<0$. This contradicts the fact that $\theta$ leads to the efficient outcome for every possible realization of types. Thus $\theta$ is continuous at $\frac{1}{\delta}$, and for the same reason, it must be continuous at $\delta$. We conclude that $\theta$ is continuous on $T=\left[\delta, \frac{1}{\delta}\right]$.

Characterization: We fix an efficient equilibrium $\theta$ and an arbitrary pair of values $\left(y^{\prime}, \tilde{y}\right) \in\left(\delta, \frac{1}{\delta}\right)^{2}$, such that $y^{\prime}<1$ and $\tilde{y}>1$. Consider now a $\mathbf{t} \in\left(\delta, \frac{1}{\delta}\right)^{n}$ such that $t_{1}=y^{\prime}$, $t_{2}=\tilde{y}, t_{3}=\frac{1}{y^{\prime} \tilde{y}} \in\left(\frac{1}{\tilde{y}}, \frac{1}{y^{\prime}}\right) \subset\left(\delta, \frac{1}{\delta}\right)$, and $t_{i}=1$ for every $i>3$. If we define $r=y^{\prime} \times \tilde{y}$ we get $\sum_{i \in N-\{1,2,3\}} \theta(1)+\theta\left(y^{\prime}\right)+\theta\left(\frac{r}{y^{\prime}}\right)+\theta\left(\frac{1}{r}\right)=0$. Since, $\ln (y)+\ln \left(\frac{r}{y}\right)+\ln \left(\frac{1}{r}\right)=0$ for every $y$ in an open ball around $y^{\prime}$, and since $\theta$ is differentiable at $y^{\prime}$, it follows that we can take the derivative of $\sum_{i \in N-\{1,2,3\}} \theta(1)+\theta(y)+\theta\left(\frac{r}{y}\right)+\theta\left(\frac{1}{r}\right)=0$ with respect to $y$ and evaluate it at $y^{\prime}$. By doing that, we get, $\theta^{\prime}\left(y^{\prime}\right)+\theta^{\prime}\left(\frac{r}{y^{\prime}}\right)\left(-\frac{r}{y^{\prime 2}}\right)=0$. This can be written as $y^{\prime} \times \theta^{\prime}\left(y^{\prime}\right)=\tilde{y} \times \theta^{\prime}(\tilde{y})$. But since this holds for any pair of values $\left(y^{\prime}, \tilde{y}\right) \in$ $\operatorname{int}(T)^{2}$, such that $y^{\prime}<1$ and $\tilde{y}>1$, it is true that, for any fixed $\tilde{y} \in\left(1, \frac{1}{\delta}\right)$, we have $y \times \theta^{\prime}(y)=\tilde{y} \times \theta^{\prime}(\tilde{y})$ for every $y \in(\delta, 1)$. In other words, for every $y \in(\delta, 1)$ we have $y \times \theta^{\prime}(y)=c \Longrightarrow \theta^{\prime}(y)=\frac{c}{y} \Longrightarrow \theta(y)=c \ln y+\hat{c}$, for some $c>0$ and $\hat{c} \in \mathbb{R}$. By the fact that $\theta(1)=0$, it follows that $\hat{c}=0$ and, hence, $\theta(y)=c \ln y$ for every $y \in(\delta, 1)$, with $c>0$. By symmetry of $\theta$ it follows that for every $y \in\left(1, \frac{1}{\delta}\right)$, we have $\theta(y)=-\theta\left(\frac{1}{y}\right)=-c \ln \frac{1}{y}=c \ln y$. That is, $\theta(y)=c \ln y$ for every $y \in\left(\delta, \frac{1}{\delta}\right)$, with $c>0$, and by continuity at the boundary, the formula must hold for every $y \in\left[\delta, \frac{1}{\delta}\right]$.

By the fact that in every equilibrium $\sigma$ of the rule $V^{1 S 1 V-D}(d)$, we must have $\sigma_{i}\left(\frac{1}{\delta}\right) \leq d_{i}$ and $\sigma_{i}(\delta) \geq-d_{i}$, and by the above analysis, it follows that in an efficient equilibrium it should hold that $\sigma_{i}\left(t_{i}\right)=c \ln t_{i}+\kappa_{i}$ with $\sum_{i \in N} \kappa_{i}=0$ and $c \in\left(0, \min _{i \in N}\left\{\frac{\min \left(d_{i}-\kappa_{i}, d_{i}+\kappa_{i}\right)}{-\ln (\delta)}\right\}\right]$.

Proof of Proposition 3. Let $X$ be a continuous voting rule: there exists $\left(\psi_{i}\right)_{i \in N} \in$ $\times_{i \in N} \operatorname{int}\left(X_{i}\right)$ such that $\sum_{i \in N} \psi_{i}=0$. To see why $X$ admits an efficient equilibrium, notice that any efficient equilibrium $\sigma$ of the voting rule $V^{1 S 1 V-D}(1, \ldots, 1)$, as characterized in Proposition 2, can be properly re-scaled so that, for each $i \in N, \sigma_{i}$ fits within any open set around $\psi_{i}$.

To understand why only continuous voting rules admit an efficient equilibrium, let $v=X$ be a voting rule with an efficient equilibrium $\sigma$. Since every strategy that is feasible according to this rule is also feasible under the rule $v^{\prime}=(\mathbb{R}, \mathbb{R}, \ldots, \mathbb{R})$, it must be 
the case that $\sigma$ is an efficient equilibrium of $v^{\prime}$ too. We replicate the reasoning in the proof of Proposition 2, and get the following result: as $\sigma$ is an efficient equilibrium of $v^{\prime}$, it should hold that $\sum_{i \in N} \sigma_{i}(1)=0$ and, for every $i \in N, \sigma_{i}(y)$ should be continuous and strictly increasing in an open ball around $y=1$. Hence, noting $\psi_{i}=\sigma_{i}(1)$, we have that $\sum_{i \in N} \psi_{i}=0$ and $\psi_{i} \in \operatorname{int}\left(X_{i}\right)$ for every $i \in N$, that is $X$ is a continuous voting rule.

\section{Appendix B: Proofs of Section 5}

Proof of Proposition 4. The result is straightforward when the manager is perfectly informed, i.e. $T_{M}=\{0, \infty\}$. In that case, the aligned manager vetoes in the bad state, while all other manager's types never veto. The setting is thus equivalent to a voting game with fixed prior $\operatorname{Pr}(\alpha)=\frac{1}{1+\mu}$, for which the dominance of $1 P 1 V$ - $D$ over $1 P 1 V$ has already been established. In the rest of the proof, we thus focus on the case where $T_{M}$ is compact

As for Proposition 1, we divide the statement in two lemmas (Lemma 7 and 8 in this Appendix), focusing respectively on the best and the worst equilibria under each voting mechanism. Throughout the proof, we treat the game between the manager and the shareholders as a simultaneous game and we continue to apply the equilibrium notion of a BNE, as in Section 4. We note however that the same results hold for the equilibrium concept of weak perfect Bayesian equilibrium in the sequential version of the game when there is no decisive shareholder.

Lemma 7 For any $\mu \in[0,1]$ and any share distribution d, for any BNE $\sigma$ under $V^{1 P 1 V}(d)$, there exists a BNE $\sigma^{\prime}$ under $V^{1 P 1 V-D}(d)$ that makes all shareholders (weakly) better off in expectation.

Proof. The strategy of the proof is to construct a two-player common value game between an aligned manager and an aggregate shareholder (holding all the shareholders' signals). When voting under $1 P 1 V$ - $D$, shareholders can implement (i.e. decentralize) the aggregate shareholder's strategy of the most efficient equilibrium of the two-player game. The corresponding profile is then the most efficient equilibrium of the original game under $1 P 1 V$ - D. Using the argument of Mc Lennan (1998), shareholders' welfare at equilibrium cannot improve with $1 P 1 \mathrm{~V}$.

Two-player game. We consider a game with two players: a manager $M$ and an aggregate shareholder $A S$. The manager receives the signal $s_{M}$, while the aggregate shareholder receives the signals $s_{1}, \ldots, s_{n}$. After receiving their (private) signals, players simultaneously choose to pass $\left(x_{j}=P\right)$ or to veto $\left(x_{j}=V\right)$ the proposal. The proposal is accepted with probability 1 if both players choose $P$, and it is accepted with probability 
$\mu$ if the manager vetoes while the aggregate shareholder passes. ${ }^{38}$ Both players share the same utility: for all $j \in\{M, A S\}$,

$$
\begin{aligned}
& u_{j}(A \mid \alpha)=1, u_{j}(A \mid \beta)=-1, \\
& u_{j}(B \mid \alpha)=u_{j}(B \mid \beta)=0 .
\end{aligned}
$$

Claim 1: the two-player game admits a most efficient strategy profile, which is also an equilibrium of that game. The expected utility of each player is at least 0 at this equilibrium.

We first show the existence of a most efficient equilibrium, and we start by computing players' best replies. Given a strategy $\sigma_{A S}$ for the aggregate shareholder, the expected utility difference between actions $P$ and $V$ for the manager, $\Delta u_{M}:=\mathbb{E}\left[u_{M}\left(x_{M}=P, \sigma_{A S}\right) \mid\right.$ $\left.s_{M}\right]-\mathbb{E}\left[u_{M}\left(x_{M}=V, \sigma_{A S}\right) \mid s_{M}\right]$, can be written as:

$$
\Delta u_{M}=(1-\mu) \mathbb{P}\left(\sigma_{A S}=P\right)\left(2 \mathbb{P}\left(\omega=\alpha \mid \sigma_{A S}=P, s_{M}\right)-1\right) .
$$

Indeed, the only difference between the actions $x_{M}=P$ and $x_{M}=V$ arises when the aggregate shareholder chooses to pass $\left(\sigma_{A S}=P\right)$ and the manager has control over her own veto (with probability $1-\mu$ ). Hence, playing $x_{M}=P$ is a best reply for the manager whenever $\mathbb{P}\left(\omega=\alpha \mid \sigma_{A S}=P, s_{M}\right) \geq 1 / 2$, or equivalently $\frac{\mathbb{P}\left(\omega=\alpha \mid \sigma_{A S}=P, s_{M}\right)}{\mathbb{P}\left(\omega=\beta \mid \sigma_{A S}=P, s_{M}\right)} \geq 1$. Noting $t_{M}=\frac{f_{M}\left(s_{M} \mid \alpha\right)}{f_{M}\left(s_{M} \mid \beta\right)}$ and $t_{\sigma_{A S}}=\frac{\mathbb{P}\left(\sigma_{A S}=P \mid \alpha\right)}{\mathbb{P}\left(\sigma_{A S}=P \mid \beta\right)}$, we may write $\frac{\mathbb{P}\left(\omega=\alpha \mid \sigma_{A S}=P, s_{M}\right)}{\mathbb{P}\left(\omega=\beta \mid \sigma_{A S}=P, s_{M}\right)}=t_{M} t_{\sigma_{A S}}$. We obtain that there is a cutoff $\bar{t}_{M}:=\frac{1}{t_{\sigma_{A S}}}$ such that $x_{M}=P$ is a best reply whenever $t_{M} \geq \bar{t}_{M}$, and that $x_{M}=V$ is a best reply otherwise.

Similarly, we denote the aggregate shareholder's type by $t_{A S}=\frac{\prod_{i=1}^{n} f_{i}\left(s_{i} \mid \alpha\right)}{\prod_{i=1}^{n} f_{i}\left(s_{i} \mid \beta\right)}=\prod_{i=1}^{n} t_{i}$. As for the manager, we obtain that $\Delta u_{A S}:=\mathbb{E}\left[u_{A S}\left(\sigma_{M}, x_{A S}=P\right) \mid s_{1}, \ldots, s_{n}\right]-$ $\mathbb{E}\left[u_{A S}\left(\sigma_{M}, x_{A S}=V\right) \mid s_{1}, \ldots, s_{n}\right]$, can be written as:

$$
\begin{aligned}
\Delta u_{A S}= & \left(\mathbb{P}\left(\sigma_{M}=P\right)+\mu \mathbb{P}\left(\sigma_{M}=V\right)\right) \times \\
& \left(2 \mathbb{P}\left(\omega=\alpha \mid \tilde{\mathbb{P}}\left(\sigma_{M}=P\right)=\frac{\mathbb{P}\left(\sigma_{M}=P\right)}{\mathbb{P}\left(\sigma_{M}=P\right)+\mu \mathbb{P}\left(\sigma_{M}=V\right)}, s_{1}, \ldots, s_{n}\right)-1\right),
\end{aligned}
$$

where $\tilde{\mathbb{P}}$ denotes the posterior probability once one knows that the manager has passed the reform (either because she chose to do so, $x_{M}=P$, or because she tried to veto, $x_{M}=V$, but the veto was not registered, which arises with probability $\mu$ ). Hence, as for the manager, there is a cutoff $\bar{t}_{A S}$, function of the manager's strategy $\sigma_{M}$, such that $x_{A S}=P$ is a best reply to $\sigma_{M}$ whenever $t_{A S} \geq \bar{t}_{A S}$, and that $x_{A S}=V$ is a best reply otherwise.

\footnotetext{
${ }^{38}$ In other words, the manager has incomplete control over her own veto: with probability $\mu$, she is "transformed" into a misaligned manager, who automatically passes the proposal.
} 
We have shown that the best reply of each player $j \in\{M, A S\}$ is characterized by a cutoff $\bar{t}_{j}$ above which $j$ plays $P$, and below which $j$ plays $V$. Now, observe that the players' type spaces $T_{M}=\left[\delta_{M}, \frac{1}{\delta_{M}}\right]$ and $T_{A S}=\left[\delta^{n}, \frac{1}{\delta^{n}}\right]$ are compact. Moreover, if one denotes by $g_{j}\left(t_{j} \mid \omega\right)$ the density according to which player $j$ is of type $t_{j}$ in state $\omega$, we obtain the expected utility of both players given the cutoffs $\left(\bar{t}_{M}, \bar{t}_{A S}\right)$ as:

$$
\begin{aligned}
\mathbb{E}\left[u_{j} \mid \bar{t}_{M}, \bar{t}_{A S}\right]= & \int_{\bar{t}_{A S}}^{\frac{1}{\delta^{n}}} d t_{A S} \int_{\delta_{M}}^{\frac{1}{\delta_{M}}} d t_{M}\left(\mu+(1-\mu) \mathbf{1}_{\left\{t_{M} \geq \bar{t}_{M}\right\}}\right) \times \\
& \left(\mathbb{P}(\omega=\alpha) g_{A S}\left(t_{A S} \mid \alpha\right) g_{M}\left(t_{M} \mid \alpha\right)-\mathbb{P}(\omega=\beta) g_{A S}\left(t_{A S} \mid \beta\right) g_{M}\left(t_{M} \mid \beta\right)\right) .
\end{aligned}
$$

As in each state, the conditional distributions of $t_{M}$ and $t_{A S}$ are continuous, the expected utility is continuous in both players' cutoffs. It follows that there exists an optimal couple of cutoffs $\left(\bar{t}_{M}^{*}, \bar{t}_{A S}^{*}\right)$ which maximizes the common utility. The corresponding strategy profile must thus be an equilibrium, the most efficient equilibrium, and also the most efficient strategy profile. As the strategy profile $\left(x_{M}=V, x_{A S}=V\right)$ yields an expected utility of 0 , the expected utility of the most efficient profile must be at least 0 .

Claim 2: The most efficient profile of the two-player game is replicable in the original game under $1 P 1 \mathrm{~V}-D$. In that game, the corresponding profile is both the most efficient profile such that the misaligned manager always proposes and the most efficient equilibrium.

In the original game under $1 P 1 \mathrm{~V}-D$, consider the strategy profile $\sigma^{*}$ where: the aligned manager behaves as the manager of the two-player game; the misaligned manager always proposes; the shareholders decentralize the aggregate shareholder's strategy of the twoplayer game by playing the log-strategy identified in Proposition 2.

First, observe that $\sigma^{*}$ is an equilibrium. Indeed, we know from Claim 1 that the aligned manager and the shareholders are playing optimally (if one individual shareholder could improve her utility, then the aggregate shareholder could do it as well in the twoplayer game, a contradiction). Moreover, passing is always a best reply for the misaligned manager.

Second, $\sigma^{*}$ is the most efficient profile among those for which the misaligned manager always proposes. Indeed, if there was a (strictly) more efficient such profile, then there would be a (strictly) more efficient profile than the one identified in the two-player game, a contradiction with Claim 1.

Third, assume by contradiction that there is a (strictly) more efficient equilibrium. By virtue of the previous assertion, it must be a strategy profile such that the misaligned manager proposes with probability strictly less than one. For such a strategy to be a best reply, it must be that shareholders always turn the reform down. Such a profile yields an expected utility of 0 for the shareholders (and the aligned manager), and thus cannot be 
a strict improvement over $\sigma^{*}$, since $\sigma^{*}$ yields at least 0 (applying Claim 1).

Claim 3: The original game under $1 P 1 \mathrm{~V}$ admits equilibria, but none of them is more efficient than the most efficient profile under $1 P 1 \mathrm{~V}-D$.

First, observe that the profile in which every manager's type vetoes and all shareholders vote against the proposal is an equilibrium, thus an equilibrium exists.

Second, any profile under $1 P 1 \mathrm{~V}$ for which the misaligned manager always proposes is replicable in the game under $1 P 1 \mathrm{~V}-D$, and thus cannot be (strictly) more efficient than $\sigma^{*}$ (applying Claim 2). Thus, no equilibrium under $1 P 1 \mathrm{~V}$ for which the misaligned manager always proposes can improve upon $\sigma^{*}$.

Finally, any equilibrium under $1 P 1 \mathrm{~V}$ such that the misaligned manager proposes with a probability strictly less than one must yield an expected utility of 0 for the shareholders and the aligned manager (same argument as under $1 P 1 \mathrm{~V}-\mathrm{D}$ ). Therefore, no equilibrium under $1 P 1 \mathrm{~V}$ can improve upon $\sigma^{*}$.

Lemma 8 For any $\mu \in[0,1]$ and any share distribution d, for any BNE $\sigma$ under $V^{1 P 1 V-D}(d)$, there exists a BNE $\sigma^{\prime}$ under $V^{1 P 1 V}(d)$ that makes all shareholders (weakly) worse off in expectation.

Proof. First, observe that the profile for which any manager vetoes the proposal and all shareholder vote against it is an equilibrium under $1 P 1 \mathrm{~V}$, with an expected utility of 0 for each shareholder.

Second, let $\sigma=\left(\sigma_{M}, \sigma_{1}, \ldots, \sigma_{n}\right)$ be a BNE under $1 P 1 V$ - $D$. We will show that this equilibrium yields an expected utility of at least 0 to all shareholders. If $\mathbb{P}\left(\sum_{i=1}^{n} \widehat{\sigma}_{i}\left(t_{i}\right) \geq\right.$ $0)=0$, the profile yields an expected utility of 0 (the proposal is never accepted), and the previous statement holds. We may thus focus on the case for which $\mathbb{P}\left(\sum_{i=1}^{n} \widehat{\sigma}_{i}\left(t_{i}\right) \geq 0\right)>0$. As $\sigma$ is an equilibrium, it must be that the misaligned manager always proposes. Then, by applying the same argument as in the proof of Lemma 7 (Claim 1), we obtain that the aligned manager's strategy must be weakly increasing.

Now, given the (aligned and misaligned) managers' strategies, the game among shareholders can be seen as a game with an exogenous proposal (as in Section 4), albeit with a possibly biased prior $\mathbb{P}(\omega=\alpha) \geq 1 / 2$. In that game, we can apply the same reasoning as in the proof of Lemma 4 (Claim 1), and we obtain that there exists a strategy profile $\sigma^{\prime}$ for the shareholders, such that each $\sigma_{i}^{\prime}$ is pure, weakly increasing and: $\forall i \in N, u_{i}\left(\sigma_{M}, \sigma_{1}, \ldots, \sigma_{n}\right)=u_{i}\left(\sigma_{M}, \sigma_{1}^{\prime}, \ldots, \sigma_{n}^{\prime}\right)$. As each player's strategy under the profile $\left(\sigma_{M}, \sigma_{1}^{\prime}, \ldots, \sigma_{n}^{\prime}\right)$ is weakly increasing, a similar argument as the one used in Lemma 5 (Claim 1) shows that this profile yields an expected utility of at least 0 to all shareholders. Therefore, $\forall i \in N, u_{i}(\sigma) \geq 0$. This concludes the proof. 
The dominance statement in Proposition 4 is obtained by conjunction of Lemmas 7 and 8. To establish strict dominance, consider an instance for which, when the proposal is exogenous, the best BNE under $1 P 1 V-D, \sigma^{1 P 1 V-D}$, implements the correct outcome with a probability $p^{1 P 1 V-D}$ strictly higher than $p^{1 P 1 V}$, attained at the best BNE under $1 P 1 V, \sigma^{1 P 1 V}$. We may further assume $p^{1 P 1 V-D}>p^{1 P 1 V}>\frac{1}{2}$. We will establish the strict dominance of $1 P 1 \mathrm{~V}$-D over $1 P 1 \mathrm{~V}$ when the proposal is endogenous by taking $\delta_{M}$ sufficiently close to 1 .

First, observe that for $\delta_{M}$ close enough to 1, the profile where the manager (either aligned or misaligned) always proposes and the shareholders play a BNE $\sigma$ of the exogenous proposal game, such that $p^{\sigma}>1 / 2$, must be an equilibrium. Indeed, on the shareholders' side, the game is the same as the one with an exogenous proposal since the manager always proposes. On the aligned manager's side, the utility of vetoing is 0 , while the utility of proposing can be made arbitrarily close to $2 p^{\sigma}-1>0$ (for $\delta_{M}$ close to 1 ), in which case proposing is indeed a best reply. Finally, as the probability of accepting the reform is always positive (since $p^{\sigma}>1 / 2$ ), the misaligned manager's best reply is also to propose.

Second, assume that there is a BNE $\sigma$ under $1 P 1 \mathrm{~V}$ which implements the correct outcome with a probability $p^{\sigma}>p^{1 P 1 V}$. As $p^{\sigma}>1 / 2$, the misaligned manager always proposes (for $\delta_{M}$ close enough to 1 ) . As $p^{1 P 1 V}$ is the highest probability to implement the correct outcome under $1 P 1 \mathrm{~V}$ when the proposal is exogenous, it must be that the aligned manager sometimes vetoes the proposal, for some type $t_{M} \in T_{M}$. It must thus be that $\mathbb{P}\left(\alpha \mid t_{M}\right) \mathbb{P}(O=A \mid \alpha)+\mathbb{P}\left(\beta \mid t_{M}\right) \mathbb{P}(O=B \mid \beta) \leq \frac{1}{2}$, where $\mathbb{P}(O \mid \omega)$ denotes the probability of alternative $O$ passing in state $\omega$ when the proposal is passed to the shareholders. By choosing $\delta_{M}$ sufficiently close to 1 , we can make $\mathbb{P}\left(\alpha \mid t_{M}\right)$ and $\mathbb{P}\left(\beta \mid t_{M}\right)$ arbitrarily close to $1 / 2$ for all $t_{M} \in\left[\delta_{M}, \frac{1}{\delta_{M}}\right]$, and, as $p^{1 P 1 V}>\frac{1}{2}$, we can thus make sure that

$$
\begin{aligned}
&\left(\exists t_{M} \in T_{M}, \quad \mathbb{P}\left(\alpha \mid t_{M}\right)\right.\left.\mathbb{P}(O=A \mid \alpha)+\mathbb{P}\left(\beta \mid t_{M}\right) \mathbb{P}(O=B \mid \beta) \leq \frac{1}{2}\right) \\
& \Rightarrow \quad\left(\forall t_{M} \in T_{M}, \quad \mathbb{P}\left(\alpha \mid t_{M}\right) \mathbb{P}(O=A \mid \alpha)+\mathbb{P}\left(\beta \mid t_{M}\right) \mathbb{P}(O=B \mid \beta)<p^{1 P 1 V}\right) .
\end{aligned}
$$

If $\sigma_{M}$ denotes the aligned manager's strategy, we obtain:

$$
\begin{aligned}
p^{\sigma}=\int\left(\mu+(1-\mu) \sigma_{M}\left(t_{M}\right)(P)\right) \times & \\
& \left(\mathbb{P}\left(\alpha \mid t_{M}\right) \mathbb{P}(O=A \mid \alpha)+\mathbb{P}\left(\beta \mid t_{M}\right) \mathbb{P}(O=B \mid \beta)\right) g_{M}\left(t_{M}\right) d t_{M}<p^{1 P 1 V} .
\end{aligned}
$$

Hence a contradiction. We have shown that for $\delta_{M}$ close enough to 1 , the dominance of $1 P 1 \mathrm{~V}-\mathrm{D}$ over $1 \mathrm{P} 1 \mathrm{~V}$ can be strict.

Proof of Lemma 3. The expected payoff of the misaligned manager when she calls for a vote in the bad state is $p_{I}\left(\gamma_{V}\right)-c\left(1-p_{I}\left(\gamma_{V}\right)\right)$. This has to be compared to a payoff 
of 0 if she vetoes the proposal. As a result, the manager strictly prefers to call for a vote if $p_{I}\left(\gamma_{V}\right)>\frac{c}{1+c}$, and is indifferent between vetoing and putting the proposal to a vote if $p_{I}\left(\gamma_{V}\right)=\frac{c}{1+c}$. Given that the misaligned manager cannot veto with probability 1 in equilibrium, it must be that $p_{I}\left(\gamma_{V}^{*}\right) \geq \frac{c}{1+c}$.

Given that $p_{I}\left(\gamma_{V}\right)$ is (weakly) increasing in $\gamma_{V}$, we have that $p_{I}(0)>\frac{c}{1+c}$ guarantees that $p_{I}\left(\gamma_{V}\right)>\frac{c}{1+c} \forall \gamma_{V} \in(0,1)$. Hence, the unique equilibrium must be such that the misaligned manager does not veto in the bad state, i.e., $\gamma_{V}^{*}=0$. It follows that the manager neither vetoes in the good state. By contrast, when $p_{I}(0)<\frac{c}{1+c}, \gamma_{V}^{*}=0$ is not an equilibrium. Given that $p_{I}\left(\gamma_{V}\right) \rightarrow 1$ when $\gamma_{V} \rightarrow 1$, we have that there must be a value of $\gamma_{V}^{*} \in(0,1)$ such that $p_{I}\left(\gamma_{V}^{*}\right)=\frac{c}{1+c}$. This pins down the equilibrium behavior of the misaligned manager in the bad state.

Proof of Proposition 5. First remember that, when perfectly informed, (i) the aligned manager always vetoes the reform in the bad state, and (ii) in the good state, the manager behaves in the same way whether aligned or misaligned. We also know that the equilibrium must be such that the manager, whether aligned or misaligned, never vetoes in the good state. So, we need to prove that there is a $c$ sufficiently small such that the misaligned manager never vetoes in the bad state.

When the misaligned manager never vetoes in the bad state, the probability of a type$I$ error in that state is positive, i.e., $p_{I}(0)>0$. This is because shareholders are then uncertain about the state of nature at the time of the meeting and hence make mistakes of both types. Thus, there must be a $c$ sufficiently small such that $p_{I}(0)>\frac{c}{1+c}$. Given that this later condition guarantees that not vetoing is a best response for the misaligned manager in the bad state, we have that there must be a $c$ sufficiently small such that the misaligned manager vetoes with probability 0 in the bad state. Given that the manager behaves in the same way under $1 P 1 \mathrm{~V}-\mathrm{D}$ and $1 \mathrm{P} 1 \mathrm{~V}$, the comparison of these two voting mechanisms depends exclusively on their voting efficiency. We know from Proposition 1 that $1 P 1 \mathrm{~V}-D$ has a higher voting efficiency than $1 P 1 \mathrm{~V}$. Thus, $1 P 1 \mathrm{~V}-\mathrm{D}$ dominates $1 P 1 \mathrm{~V}$ when $c$ is sufficiently small.

\section{Appendix C: Numerical Examples for Section 4}

Consider a firm with six shareholders. Shareholders 1, 2, and 3 hold $k \in\{1,2,3\}$ shares each, while shareholders 4,5 , and 6 hold $4-k$ shares each. Signals are binary, $s_{i} \in\{a, b\}$, and the likelihood of receiving the right signal is $p(a \mid \alpha)=p(b \mid \beta)=$ $p_{H} \in[.55,1)$ for shareholders $1-3$, and $p(a \mid \alpha)=p(b \mid \beta)=.55$ for shareholders 


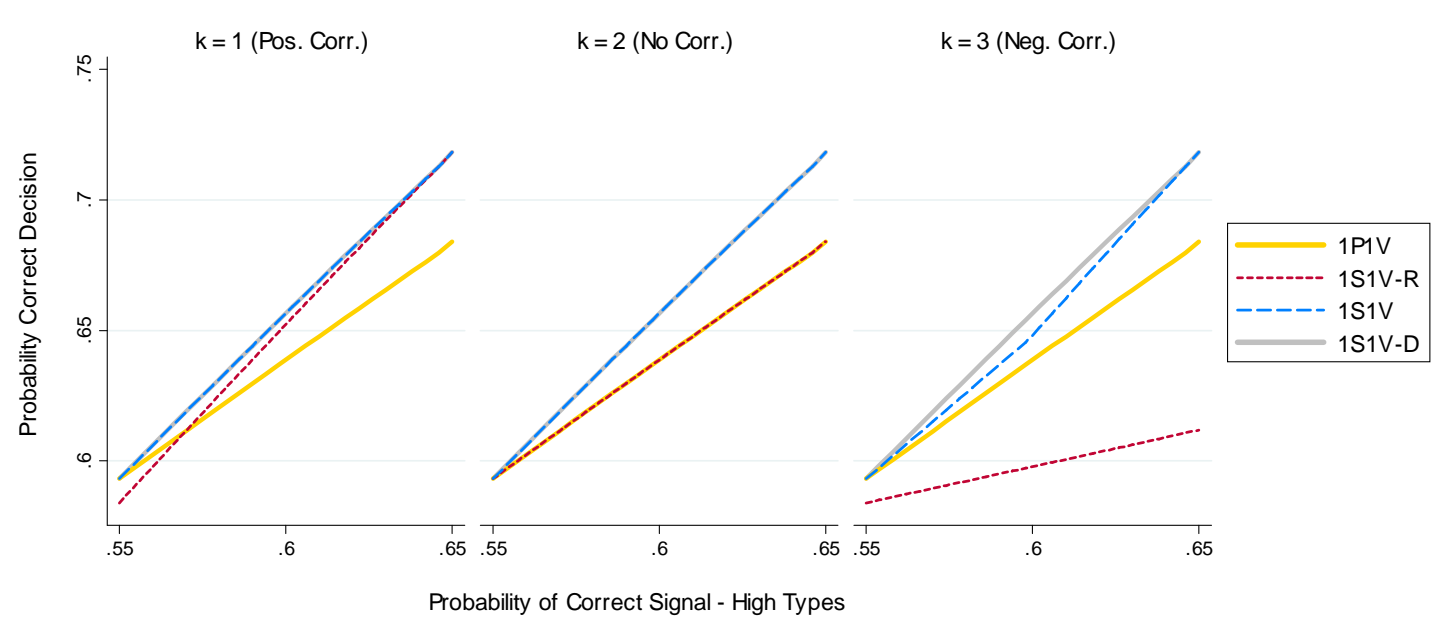

Figure 4: Probability of a correct decision under 1P1V, 1S1V-R, 1S1V and 1S1V-D.

$4-6 .{ }^{39}$ That is, shareholders $1-3$ have more accurate information -they estimate correctly the true state of the world with a probability $p_{H} \geq 0.55-$ while the other three shareholders are less accurately informed -they correctly infer the true state of the world with a probability 0.55 . Figure 4 plots the probability of taking the right decision under $1 S 1 \mathrm{~V}, 1 S 1 \mathrm{~V}-\mathrm{R},{ }^{40}$ and $1 \mathrm{P} 1 \mathrm{~V}$ (and $1 S 1 \mathrm{~V}$-D as the efficient benchmark, that will be discussed later).

As we know from Lemma 1, a key to aggregate information efficiently is to attach more weight to more informative signals. In our simple example, shareholders $1-3$ are always the ones that have more informative signals, and therefore their signals should have more weight in the group decision. Under $1 P 1 \mathrm{~V}$, however, all votes have the same weight and this undermines the ability of shareholders to aggregate information well, especially compared to $1 S 1 \mathrm{~V}$. Under $1 P 1 \mathrm{~V}$ there are two types of equilibria: (i) an equilibrium where all shareholders vote their signal and (ii) an equilibrium in which two of the lowly informed shareholders mute themselves by voting for opposite options. Each type of equilibria has a different type of inefficiencies: in the former, the cost is that lowly informed voters overwrite decisions by the highly informed while in the second some of the information by the lowly informed

\footnotetext{
${ }^{39}$ These parameters would be equivalent to having $f_{i}\left(s_{i} \mid \alpha\right)=f_{i}\left(1-s_{i} \mid \beta\right)=2 p$ for every $s_{i}<\frac{1}{2}$ and $f_{i}\left(s_{i} \mid \alpha\right)=f_{i}\left(1-s_{i} \mid \beta\right)=2(1-p)$ for every $s_{i} \geq \frac{1}{2}$, where $p=p_{H}$ for shareholders $1-3$ and $p=.55$ for shareholders $4-6$.

${ }^{40}$ The one-share-one-vote without partial abstention mechanism, $V^{1 S 1 V-R}$, is such that $V^{1 S 1 V-R}(d)=\times_{i \in N}\left\{-d_{i}, d_{i}\right\}$. This mechanism has the same power imbalance as $1 S 1 \mathrm{~V}$ but a less richer ballot space since shareholders cannot partially abstain.
} 
shareholders is lost.

Under $1 S 1 \mathrm{~V}$-R, shareholders votes have weights proportional to their shares. The ability of this mechanism to aggregate information critically depends on the correlation between the number of shares and the quality of shareholders' information. When $k=2$, all shareholders hold the same number of shares, and $1 S 1 \mathrm{~V}-\mathrm{R}$ mimics the properties of $1 \mathrm{P} 1 \mathrm{~V}$. When $k=1$, there is a positive correlation between shares and quality of information, and in this case $1 S 1 \mathrm{~V}-\mathrm{R}$ outperforms $1 \mathrm{P} 1 \mathrm{~V}$ only if shareholders with more shares are sufficiently better informed. When $k=3$, there is a negative correlation between shares and quality of information, and in this case it is $1 P 1 \mathrm{~V}$ that outperforms $1 S 1 \mathrm{~V}$-R.

Finally, as one can see from Figure $4,1 S 1 \mathrm{~V}$ dominates both $1 \mathrm{P} 1 \mathrm{~V}$ and $1 S 1 \mathrm{~V}-\mathrm{R}$. The key for that result is that voters can endogenously determine the weight of their vote. This allows the group's decision to depend more heavily on more informative signals. The difference is particularly significant in cases with negative correlation between shares and information $(k=1){ }^{41}$

Despite the superiority of $1 S 1 \mathrm{~V}$, it may not be fully efficient (as one can see in the case of $k=3$ in Figure 4). The sources of inefficiency of $1 S 1 \mathrm{~V}$ are the discreteness of the action space and, perhaps more importantly, the potential mismatch between the number of shares own by a shareholder and the quality of her information. When $k=3$ in the previous example, $1 S 1 \mathrm{~V}$ is not efficient because better informed shareholders have too few shares in order to reveal their information through the voting process.

\section{Appendix D: Extensions}

In this Appendix, we explore the robustness of the results in Section 4 when allowing for partisan shareholders, and when endogenizing the acquisition of information by shareholders.

\section{Partisans}

There is evidence that disagreement among shareholders may not only stem from information asymmetries, but also from differences in preferences (Bolton et al., 2020). As mentioned in Cvijnovic et al. (2019, p.3), preferences may vary due

\footnotetext{
${ }^{41}$ It is worth pointing out that in the more general setup, it is not ex-ante clear which subjects will receive the most informative signal, and this makes $1 S 1 \mathrm{~V}$ - $R$ relatively worse.
} 
to differences in, e.g., portfolio allocation (Cohen and Schmidt 2009), business ties (Davis and Kim 2007, and Cvijnovic et al. 2016), reputational concerns (Chevalier and Ellison 2009), and political and social goals (Woidtke 2002).

We consider the same model as in Section 2 except that we allow for three types of shareholders: $A, B$, and $C$. The utility of the different shareholders are given by:

$$
\forall O \in\{A, B\}, \forall \omega \in\{\alpha, \beta\}, \quad\left\{\begin{array}{l}
u_{i}^{A}(O \mid \omega)=h_{i} \times \mathbf{1}_{\{O=A\}} \\
u_{i}^{B}(O \mid \omega)=h_{i} \times \mathbf{1}_{\{O=B\}} \\
u_{i}^{C}(O \mid \omega)=h_{i} \times\left(\mathbf{1}_{\{O=A, \omega=\alpha\}}-\mathbf{1}_{\{O=A, \omega=\beta\}}\right) .
\end{array}\right.
$$

That is, $A$ (resp. $B$ )-shareholders want $A$ (resp. $B$ ) to be selected irrespective of the state $\omega$, whereas $C$-shareholders are common-value shareholders who want the decision to match the state. This way of modeling partisan shareholders is in line with Bar-Isaac and Shapiro (2020).

We denote the fraction of shares held by $A$ (resp. $B$ )-shareholders by $h_{A}$ (resp. $\left.h_{B}\right)$. Common-value shareholders own the remaining fraction of the stock, $h_{C}=1-$ $h_{A}-h_{B}$. Only $C$-shareholders receive a signal about the quality of the proposal. ${ }^{42}$ In what follows, we consider two different cases: (i) $h_{A}$ and $h_{B}$ are common knowledge, and (ii) $h_{A}$ and $h_{B}$ are not common knowledge.

Partisan shareholders ( $A$ and $B$ types) are always (weakly) better off by giving the largest possible number of votes to their favorite alternative, independently of the behavior of the other shareholders (as in Nunez and Laslier 2014). To ensure that our results do not hinge on possible beliefs that make partisan voters indifferent between several actions, we consider that such voters always play that unique dominant strategy.

An implication of partisan voters' dominant strategy is that if different mechanisms allow for a different maximum number of votes for different shareholders, partisans will have an asymmetric effect across mechanisms. For this reason, we limit attention to comparing voting mechanisms which result in the same distribution of power:

Definition 7 If two mechanisms, $V$ and $V^{\prime}$, with associated rules $V(d)=X$ and $V^{\prime}(d)=X^{\prime}$, are such that $\max X_{i}=\max X_{i}^{\prime}$ and $\min X_{i}=\min X_{i}^{\prime}$ for every shareholder $i$ and any distribution of shareholdings $d$, then $V$ assigns power similarly to $V^{\prime}$.

\footnotetext{
${ }^{42}$ Allowing partisan shareholders to be informed does not alter the results, but requires additional notation.
} 
That is, while different shareholders might have a different maximum number of votes, each shareholder must have the same maximum number of votes under any mechanism that we compare.

Finally, while the game now includes non-common value shareholders, we still use the notion of dominance and efficiency defined in Section 3. The main reason to do so is that our focus is on the informational efficiency of voting mechanisms. Another reason is that these welfare benchmarks, even if they are better suited for the analysis of common-value elections, also admit a utilitarian interpretation in certain contexts with heterogeneous preferences. ${ }^{43}$

\section{Partisan Shareholders are Common Knowledge.}

When there is no uncertainty about the fraction of shares held by partisans, all our main results mostly hold. In particular, we can prove that (i) mechanisms with richer ballot spaces dominate mechanisms with poorer ballot spaces if these mechanisms do not differ in how they distribute power accross shareholders, and (ii) the $1 S 1 \mathrm{~V}$ - $\mathrm{D}$ mechanism and other continous mechanisms are efficient and their superiority over other mechanisms might be reinforced.

Proposition 7 Consider that $h_{A}$ and $h_{B}$ are common knowledge. Under Assumption 1: (i) if a finite mechanism $V$ has a richer ballot space than mechanism $V^{\prime}$ -but the two mechanisms distribute power similarly-and $d$ is such that there is no decisive voter, then $V$ dominates $V^{\prime}$; and (ii) if $d$ is such that $h_{C}>\left|h_{A}-h_{B}\right|$, then $V^{1 S 1 V-D}$, admits an efficient BNE.

Proof. In this proof, we denote by $N_{A}$ (resp. $N_{B}$ ) the set of $A$-partisans (resp. $B$ partisans) and by $N_{C}$ the set of common value voters.

(i) for any distribution $d$, we may write $V(d)=X$ and $V^{\prime}(d)=X^{\prime}$ with for all $i \in N$, $X_{i} \subseteq X_{i}^{\prime}$. The proof's strategy consists in re-writing the voting games associated to

\footnotetext{
${ }^{43}$ When there are partisan shareholders, the correct outcome (i.e., $A$ in state $\alpha$, and $B$ in state $\beta$ ) does not necessarily coincide with the utilitarian one. Under $1 S 1 \mathrm{~V}$ and $1 S 1 \mathrm{~V}$ - $D$, when partisans have so many votes that common-value shareholders cannot affect the final outcome, then in one state the correct outcome coincides with the one that maximizes the sum of utilities; and in the other state they differ. Importantly, those situations are the same for $1 S 1 \mathrm{~V}$ and $1 S 1 \mathrm{~V}$-D. In the remaining situations, when the behavior of the common-value shareholders matters for the final outcome, the correct outcome coincides with the utilitarian one. Hence, if one of the two voting mechanisms is found to admit a BNE that implements the correct outcome with higher probability than any BNE of the other mechanisms, then it follows that it also implements the utilitarian outcome with higher probability.
} 
these rules as voting games taking place among common value shareholders, and to apply Proposition 1.

For a C-shareholders' vote profile $\mathbf{x}=\left(x_{i}\right)_{i \in N_{C}}$, a proposal passes (for sure) if and only if:

$$
\sum_{i \in N_{A}}\left(\max X_{i}\right)+\sum_{i \in N_{B}}\left(\min X_{i}\right)+\sum_{i \in N_{C}} x_{i}>0 \Leftrightarrow \sum_{i \in N_{C}}\left(x_{i}+\frac{\sum_{i \in N_{A}}\left(\max X_{i}\right)+\sum_{i \in N_{B}}\left(\min X_{i}\right)}{\# N_{C}}\right)>0 .
$$

For $i \in C$, we let $Y_{i}=\left\{x_{i}+\frac{\sum_{i \in N_{A}}\left(\max X_{i}\right)+\sum_{i \in N_{B}}\left(\min X_{i}\right)}{\# N_{C}} \mid x_{i} \in X_{i}\right\}$ and $Y_{i}^{\prime}=$ $\left\{x_{i}+\frac{\sum_{i \in N_{A}}\left(\max X_{i}\right)+\sum_{i \in N_{B}}\left(\min X_{i}\right)}{\# N_{C}} \mid x_{i} \in X_{i}^{\prime}\right\}$. By application of Proposition 1, the rule $Y^{\prime}$ dominates the rule $Y$, it follows from the previous equivalence that we also have that $X^{\prime}$ dominates $X$.

(ii) for any $d$ such that $h_{C}>\left|h_{A}-h_{B}\right|$, an efficient equilibrium can be constructed under $V^{1 S 1 V-D}(d)$, following the same argument as the one described in the main text (re-scaling of the efficient equilibrium of Proposition 2).

To understand this result, let us first focus on the efficiency of $1 S 1 \mathrm{~V}$-D. Shareholder $i$ will choose $+d_{i}$ if she is a $A$-shareholder, and $-d_{i}$ if she is a $B$-shareholder. When $h_{A}$ is larger than $h_{B}$, it is as if the voting mechanism were biased against the status quo, and conversely for $h_{A}<h_{B}$. To implement the efficient outcome, $C$ shareholders must then find a way to compensate for that bias. An easy way to do so is for common-value shareholders to make two modifications to the efficient equilibrium strategy under $1 S 1 \mathrm{~V}-\mathrm{D}$ as characterized in Proposition 2. First, common-value shareholders have to rescale their strategy by a factor of $\left(1-\frac{\left|h_{A}-h_{B}\right|}{h_{C}}\right)$ to leave room for compensation. Second, if say $h_{B}>h_{A}$, each common-value shareholder $i$ includes $d_{i} \times \frac{h_{B}-h_{A}}{h_{C}}$ points in favor of $A$ on her ballot.

For instance, consider a case with ten common-value shareholders holding 10 shares each, and partisan shareholders, all of them of type $B$, holding cumulatively 20 shares. Assume that, without the partisans, each common-value shareholder would give 10 points to $A$ when receiving the most informative signal in favor of $A, 2$ points to $A$ when receiving another, less informative signal in favor of $A$, and -5 points to $A$ (i.e. 5 points to $B$ ) when receiving a signal in favor of $B$. With partisan shareholders, each common-value shareholder would rescale her strategy by a factor of 0.8 (i.e., giving 0.8 times the number of points she would have given without partisan shareholders), and add 2 points for $A$. Thus, she would still give $10(=10 \times 0.8+2)$ points to $A$ when receiving the most informative signal in favor of $A$, but she would now give $3.6(=2 \times 0.8+2)$ points to $A$ when receiving the less 
informative signal in favor of $A$, and $-2(=-5 \times 0.8+2)$ points to $A$ (i.e. 2 points to $B$ ) when receiving a signal in favor of $B$.

\section{Partisan Shareholders are Not Common Knowledge.}

To explore this case, we assume that the distribution of shares $d=\left(d_{i}\right)_{i \in N}$ is common knowledge among shareholders, but the partisan type of each shareholder is unknown. Specifically, the partisan type of each shareholder $i \in N$ is drawn from a distribution $p_{i} \in \Delta(\{A, B, C\})$, and her utility is given by $(3)$. We let $p=\left(p_{i}\right)_{i \in N}$ denote the shareholder-specific partisan-type distribution.

In this extended model, we show that mechanisms with richer ballot spaces still dominate mechanisms with poorer ballot spaces (provided that they distribute power similarly) when we restrict our attention on their best equilibria.

Proposition 8 Consider two finite mechanisms $V$ and $V^{\prime}$, a distribution of shareholdings $d$, and a partisan-type distribution $p$. Under Assumptions 1, if $V$ has a richer ballot space than mechanism $V^{\prime}$-but the two mechanisms distribute power similarly-, then for any BNE under $V^{\prime}(d)$ there exists a BNE under $V(d)$ such that the probability of making the correct decision is higher.

Proof. Let $X$ be a finite voting rule. The voting game can then be re-written as one of common values. In this game, each shareholder $i \in N$ has utility $u_{i}^{C}$ (we then have common values in the sense that $u_{i}^{C}=\frac{h_{j}}{h_{i}} u_{j}^{C}$ ). When she chooses an action $x_{i} \in X_{i}$, the action is recorded with probability $p_{i}^{C}$, while it is transformed into $\left(\max X_{i}\right)$ with probability $p_{i}^{A}$ and into $\left(\min X_{i}\right)$ with probability $p_{i}^{B}$.

The existence of a BNE for that game is obtained by the same argument as in the proof of Lemma 4. The only difference arises with the expressions (in the proof of Claim 1) $\mathbb{P}_{\sigma_{-i}}\left(\sum_{j \neq i} x_{j}>-x \mid \omega\right)+\frac{1}{2} \mathbb{P}_{\sigma_{-i}}\left(\sum_{j \neq i} x_{j}=-x \mid \omega\right)$ which should be replaced by $\mathbb{P}_{\tilde{\sigma}_{-i}}\left(\sum_{j \neq i} x_{j}>-x \mid \omega\right)+\frac{1}{2} \mathbb{P}_{\tilde{\sigma}_{-i}}\left(\sum_{j \neq i} x_{j}=-x \mid \omega\right)$, where the strategy $\tilde{\sigma}_{j}$ is defined for each shareholder $j$ by:

$$
\tilde{\sigma}_{j}= \begin{cases}\sigma_{j} & \text { with probability } 1-p_{j}^{A}-p_{j}^{B} \\ \left(\max X_{j}\right) & \text { with probability } p_{j}^{A} \\ \left(\min X_{j}\right) & \text { with probability } p_{j}^{B} .\end{cases}
$$

We thus obtain the existence of a BNE under the finite rule $X$. To conclude, if we have two finite mechanisms $V$ and $V^{\prime}$ that distribute power similarly, then for each $d$, the common value games associated to $V(d)$ and $V^{\prime}(d)$ are such that: the action spaces of the second game are included in the action spaces of the first game (for each shareholder); the payoffs 
associated to any profile is the same in the two games (since by assumption they distribute power similarly). As before, we thus obtain that for any BNE under $V^{\prime}(d)$, there exists a BNE under $V$ such that the probability of making the correct decision is higher.

To understand this result, first note that partisan shareholders behave in the same way under $V$ and $V^{\prime}$ : they give as many points as possible to their favorite alternative. Thus, they create the same noise under the two voting mechanisms. To implement their desired outcomes, common-value shareholders have to correct for that noise while still finding a way to reveal their information. The relatively richer ballot space under $V$ than $V^{\prime}$ gives more leeway to common-value shareholders to achieve this.

While a richer ballot space remains improves information aggregation, the presence of an uncertain number of partisan voters leads to inefficiencies, even under continuous voting mechanisms. Figure 5 illustrates that point: the probability of reaching the correct decision given the collective information decreases with the frequency of partisan shareholder $B$ under $1 S 1 \mathrm{~V}$ (with a large number of votes). Note that this is also true under $1 P 1 V$. This suggests an explanation for the empirical

finding that firms with more heterogeneous shareholder base under-perform (see, e.g., Kandel et al. 2011, and Schwartz-Ziv and Volkova 2020).

\section{Endogenous Information}

Consider now that the signal is not free. Specifically, we introduce a pre-stage to the game in which shareholders independently and simultaneously decide whether to acquire an informative signal at cost $l>0$, or to stay only with the prior. In the next stage of the game, the shareholders observe who acquired a signal, and then vote. For tractability, we focus on equilibria in which shareholders use pure strategies in the first stage, and the BNE that maximizes the probability of making the correct decision in each subgame.

This is the model considered in Persico (2004), with two differences: (i) we allow for more general signal structures and a more general class of voting mechanisms, and (ii) he allows for imperfect information (i.e. shareholders do not necessarily observe who acquired a signal). This simplifying assumption helps us deal more easily with the large variety of voting rules and signals that we consider here, but we argue after the statement of the result why it is robust to having unobservable information acquisition at least as far as the comparison of best equilibria of different mechanisms is concerned. 


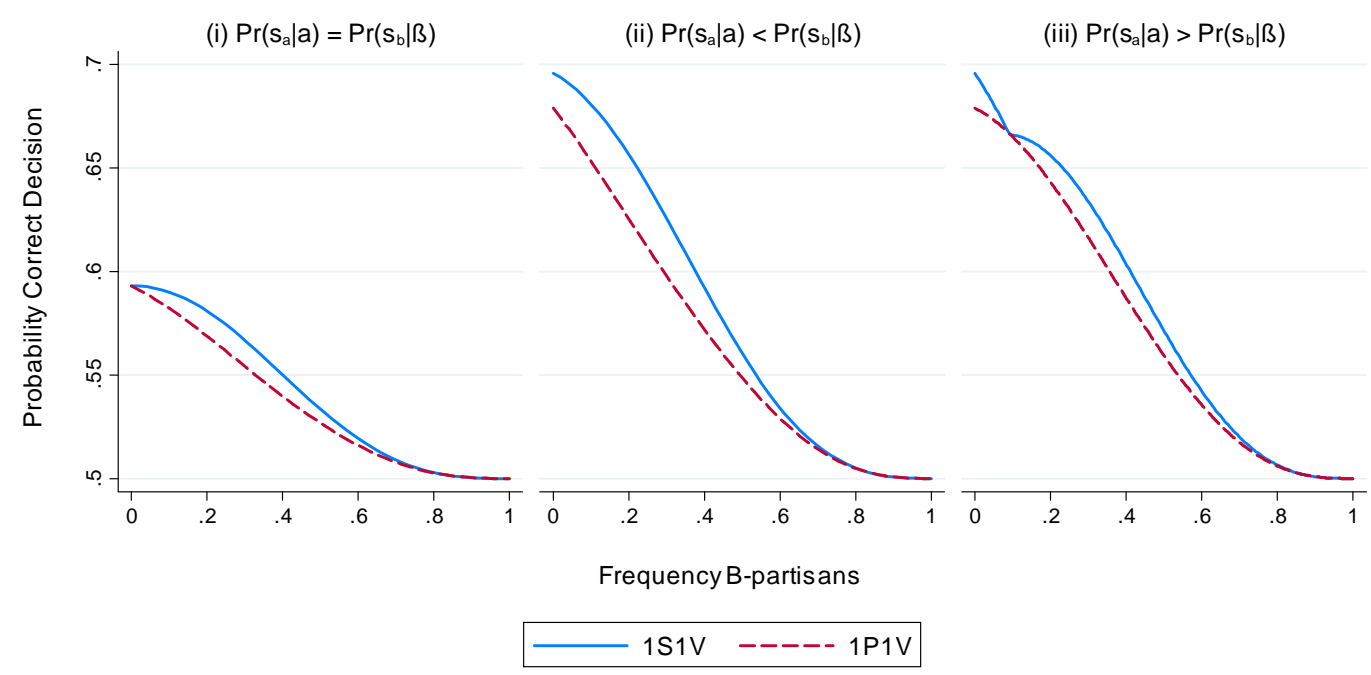

Figure 5: Probability of correct decision under 1S1V (with a large number of votes) and $1 \mathrm{P} 1 \mathrm{~V}$ with binary signals and a random number of $\mathrm{B}$ partisans. $n=6$ and priors are even. The conditional probabilities of receiving the correct signals in each state are $\operatorname{Pr}\left(s_{a} \mid \alpha\right)=\operatorname{Pr}\left(s_{b} \mid \beta\right)=55 \%$ in (i), $\operatorname{Pr}\left(s_{a} \mid \alpha\right)=55 \%$ and $\operatorname{Pr}\left(s_{b} \mid \beta\right)=65 \%$ in (ii), and $\operatorname{Pr}\left(s_{a} \mid \alpha\right)=65 \%$ and $\operatorname{Pr}\left(s_{b} \mid \beta\right)=55 \%$ in (iii).

In this setup, we can show that continuous voting mechanisms (like $1 S 1 \mathrm{~V}$-D) still dominate all the other voting mechanisms but with a different welfare criterion in mind: the probability of making the correct decision net of total information acquisition costs. It seems reasonable that shareholders should care both about the accuracy of their choice and the cost of information acquisition. Now, this does not exclude the possibility that another mechanism is superior in terms of maximizing the probability of making the correct decision independent of the information acquisition costs.

Proposition 9 Under Assumptions 1 and 2, the probability of making the correct decision net of total information costs is higher under a continuous voting mechanism compared to any other voting mechanism.

Proof. In this proof, we abuse notation and write simply $1 S 1 \mathrm{~V}-\mathrm{D}$ for the voting rule $V^{1 S 1 V-D}(d)$. A subgame is essentially defined by the set of shareholders that acquire a signal. Let us denote the set of informed shareholders by $I$. By Proposition 1 and by the argument of Mc Lennan (1998) we have that, from an ex-ante point of view, the probability of the firm making the correct decision in subgame $I$ under voting rule $v$, denoted by $\mathbb{P}(I, v)$, must satisfy $\mathbb{P}(I, v) \geq \mathbb{P}\left(I^{\prime}, v\right)$ when $\# I>\# I^{\prime}$ for any $v$, and $\mathbb{P}(I, 1 S 1 V-D) \geq$ 
$\mathbb{P}(I, v)$ for any $I$ and any $v$. We also notice that since we have fixed a certain BNE in each subgame, the whole game may be viewed as a single stage game in which the shareholders only decide whether to draw an informative signal or not. This simplified version of the game is a potential game with potential function $\mathbb{P}(I(x), v)-\# I(x) \times l$, where $x$ is the vector of information acquisition decisions with $x_{i}=1$ when shareholder $i$ acquires a signal and $x_{i}=0$ otherwise; and $\# I(x)$ is the number of informed shareholders. By the fact that there are finitely many alternative vectors $x$, the potential function obtains a maximum value for (at least) one of these vectors, which is also a pure strategy equilibrium of this simplified game (see for instance Monderer and Shapley, 1996). Moreover, every pure strategy equilibrium of this simplified game must be a maximizer of this potential function. Assume now, that a pure strategy equilibrium, $x_{1 S 1 V-D}^{*}$, exists under $1 S 1 \mathrm{~V}-D$ such that $\mathbb{P}\left(I\left(x_{1 S 1 V-D}^{*}\right), 1 S 1 V-D\right)-\# I\left(x_{1 S 1 V}^{*}\right) \times l<\mathbb{P}\left(I\left(x_{v}^{*}\right), V\right)-\# I\left(x_{v}^{*}\right) \times l$, where $x_{v}^{*}$ is an equilibrium of some other rule $v$. Since $\mathbb{P}(I, 1 S 1 V-D) \geq \mathbb{P}(I, v)$ for any $I$ and any $v$, it follows that $\mathbb{P}\left(I\left(x_{v}^{*}\right), 1 S 1 V-D\right)-\# I\left(x_{v}^{*}\right) \times l \geq \mathbb{P}\left(I\left(x_{v}^{*}\right), v\right)-\# I\left(x_{v}^{*}\right) \times l$, which contradicts the fact that $x_{1 S 1 V-D}^{*}$ is an equilibrium - and, thus, a maximizer of the corresponding potential function - under $1 S 1 \mathrm{~V}-D$. Therefore, there is no rule $v$ that admits a better equilibrium than $1 S 1 \mathrm{~V}-\mathrm{D}$.

The intuition behind this result relies on the following fact. Consider a given profile of information acquisition decisions of the other shareholders. Then, an increase in the expected utility of a shareholder if she acquires information corresponds to the increase in the probability of making the correct decision net of the increase in total information acquisition costs. Hence, the information acquisition game is a potential game. Its potential is the probability of making the correct decision net of the total information acquisition costs.

Now, let us consider shareholders using the same profile of information acquisition decisions under a continuous voting mechanism as in the equilibrium of another voting mechanism. The value of the potential corresponding to the continuous voting mechanism must be at least as high as that of the other mechanism. This follows from the superior information aggregation properties of continuous voting mechanisms. For that profile of information acquisition decisions, the information costs are the same under the two mechanisms, but the probability of making the correct decision is higher under continuous voting mechanisms. Hence, in every equilibrium under continuous voting mechanisms the value of the potential must be strictly larger compared to the value of the potential in any equilibrium of any other mechanism.

The above result is robust to information acquisition decisions being unobserv- 
able. To see this, notice that the best equilibrium of the game with observable information acquisition decisions remains an equilibrium of the game with unobservable information decisions under any voting mechanism. Indeed, under observable information acquisition, in the best equilibrium of a voting mechanism when a shareholder who is expected to acquire information does not acquire information, she decreases her expected utility (otherwise, we would not be in equilibrium), but less so compared to the case of unobservable information acquisition decisions: in the first case, all shareholders adjust and use the welfare maximizing BNE of the voting subgame, but in the latter they cannot do so and the probability of making the correct decision decreases even more. Hence, continuous voting mechanisms deliver a higher probability of making the correct choice net of total information acquisition costs, even when information acquisition decisions are unobservable. 